\title{
Synthesis of a Bulky and Electron-rich Derivative of SEGPhos and Its Application in Ru-catalyzed Enantioselective Hydrogenation of $\beta$-Ketoesters
}

Xiaobing Wan $^{\mathrm{a}}$, Yanhui Sun, ${ }^{\mathrm{b}}$ Yunfei Luo ${ }^{\mathrm{a}}$, Dao Li ${ }^{\mathrm{a}}$, and Zhaoguo Zhang ${ }^{\mathrm{a}^{*}}$

${ }^{\text {a }}$ State Key Laboratory of Organometallic Chemistry and Key Laboratory of Computer Science, Shanghai Institute of Organic Chemistry, Shanghai 200032, China

${ }^{b}$ Department of Chemistry, Nanjing University, Nanjing 210093, China

zhaoguo@mail.sioc.ac.cn

\section{Table of contents}

Page

General

Analytical and spectral data for compounds 6a-i, 8a-e

NMR Spectrum of compounds 2-4, 6a-i, 8a-e

GC data of Table1

HPLC data of Table2

S40-50

GC data of Table3

S51-56 


\section{Experimental Section}

General: All reactions were carried out under inert atmosphere of dry argon or nitrogen. THF and toluene were freshly distilled from sodium/benzophenone ketyl, while $\mathrm{CH}_{2} \mathrm{Cl}_{2}$, was distilled from $\mathrm{CaH}_{2}$ under argon atmosphere. EtOH for catalyst preparation or hydrogenation was distilled from magnesium under atmosphere. The preparation of samples and the setup of high-pressure reactor were either carried out in a glovebox or using standard Schlenk-type techniques. ${ }^{1} \mathrm{HNMR}$ (300 MHz), ${ }^{13} \mathrm{CNMR}(75.4 \mathrm{MHz})$ and ${ }^{31} \mathrm{PNMR}(121 \mathrm{MHz})$ were registered on $300 \mathrm{M}$ spectrometers with $\mathrm{CDCl}_{3}$ as solvent and tetramethylsilane (TMS) as internal standard. Chemical shifts are reported in units (ppm) by assigning TMS resonance in the ${ }^{1} \mathrm{H}$ spectrum as $0.00 \mathrm{ppm}$ and $\mathrm{CDCl}_{3}$ resonance in the ${ }^{13} \mathrm{C}$ spectrum as $77.0 \mathrm{ppm}$. All coupling constants ( $J$ values) were reported in Hertz $(\mathrm{Hz})$. Column chromatography was performed on silica gel 300-400 mesh. GC, HPLC, MS, HRMS and optical rotations were performed by the State-authorized Analytical Center in Shanghai Institute of Organic Chemistry, Chinese Academy of Science. 
3-Hydroxy-3-Phenyl-Propionic acid ethyl ester (6a) ${ }^{1} \mathrm{HNMR}\left(300 \mathrm{MHz}, \mathrm{CDCl}_{3}\right) \delta$ $1.23(\mathrm{t}, \mathrm{J}=6.9 \mathrm{~Hz}, 3 \mathrm{H}), 2.68-2.73(\mathrm{~m}, 2 \mathrm{H}), 3.56(\mathrm{br}, 1 \mathrm{H}), 4.14$ (q, 6.9Hz, 2H), 5.10 (dd, $\mathrm{J}=4.5 \mathrm{~Hz}, 8.7 \mathrm{~Hz}, 1 \mathrm{H}), 7.26-7.34(\mathrm{~m}, 5 \mathrm{H}) ;{ }^{13} \mathrm{CNMR}\left(75.4 \mathrm{MHz}, \mathrm{CDCl}_{3}\right) \delta 13.9,43.3$, $60.7,70.1,125.5,127.6,128.3,142.5,172.2$;

3-Hydroxy-3-(4-Methoxyphenyl)-Propionic Acid Ethyl Ester (6b) ${ }^{1} \mathrm{HNMR}$ $\left(300 \mathrm{MHz}, \mathrm{CDCl}_{3}\right) \delta 1.26(\mathrm{t}, \mathrm{J}=7.2 \mathrm{~Hz}, 3 \mathrm{H}), 2.64-2.81(\mathrm{~m}, 2 \mathrm{H}), 3.23(\mathrm{br}, 1 \mathrm{H}), 3.80(\mathrm{~s}$, $3 \mathrm{H}), 4.18(\mathrm{q}, \mathrm{J}=7.2 \mathrm{~Hz}, 2 \mathrm{H}), 5.06-5.11(\mathrm{~m}, 1 \mathrm{H}), 6.88(\mathrm{~d}, \mathrm{~J}=8.4 \mathrm{~Hz}, 2 \mathrm{H}), 7.30(\mathrm{~d}$, $\mathrm{J}=8.4 \mathrm{~Hz}, 2 \mathrm{H}) ;{ }^{13} \mathrm{CNMR}\left(75.4 \mathrm{MHz}, \mathrm{CDCl}_{3}\right) \delta 14.1,43.3,55.2,60.8,69.9,113,8$, $126.9,134.9,159.3,172.4$.

3-Hydroxy-3-(4-Methylphenyl)-Propionic Acid Ethyl Ester (6c) ${ }^{1} \mathrm{HNMR}(300 \mathrm{MHz}$, $\left.\mathrm{CDCl}_{3}\right) \delta 1.27(\mathrm{t}, \mathrm{J}=7.2 \mathrm{~Hz}, 3 \mathrm{H}), 2.34(\mathrm{~s}, 3 \mathrm{H}), 2.70-2.75(\mathrm{~m}, 2 \mathrm{H}), 3.25$ (br, 1H), 4.14 (q, $7.2 \mathrm{~Hz}, 2 \mathrm{H}), 5.08-5.13(\mathrm{~m}, 1 \mathrm{H}), 7.17(\mathrm{~d}, \mathrm{~J}=8.1 \mathrm{~Hz}, 2 \mathrm{H}), 7.27(\mathrm{~d}, \mathrm{~J}=8.1 \mathrm{~Hz}, 2 \mathrm{H})$; ${ }^{13} \mathrm{CNMR}\left(75.4 \mathrm{MHz}, \mathrm{CDCl}_{3}\right) \delta 14.1,21.1,43.3,60.8,70.1,125.6,129.2,137.5,139.5$, 172.4 .

3-Hydroxy-3-(4-Bromophenyl)-Propionic Acid Ethyl Ester (6d) ${ }^{1} \mathrm{HNMR}$ (300MHz, $\left.\mathrm{CDCl}_{3}\right) \delta 1.25(\mathrm{t}, \mathrm{J}=7.2 \mathrm{~Hz}, 3 \mathrm{H}), 2.64-2.70(\mathrm{~m}, 2 \mathrm{H}), 3.47(\mathrm{br}, 1 \mathrm{H}), 4.16$ (q, J=7.2Hz, $2 \mathrm{H}), 5.08(\mathrm{~m}, 1 \mathrm{H}), 7.24(\mathrm{~d}, \mathrm{~J}=7.8 \mathrm{~Hz}, 2 \mathrm{H}), 7.46(\mathrm{~d}, \mathrm{~J}=7.8 \mathrm{~Hz}, 2 \mathrm{H}) ;{ }^{13} \mathrm{CNMR}(75.4 \mathrm{MHz}$, $\left.\mathrm{CDCl}_{3}\right) \delta 14.1,43.1,61.0,69.6,121.5,127.4,131.6,141.4,172.2$.

3-Hydroxy-3-(4-Chlorophenyl)-Propionic Acid Ethyl Ester (6e) ${ }^{1} \mathrm{HNMR}(300 \mathrm{MHz}$, $\left.\mathrm{CDCl}_{3}\right) \delta 1.27(\mathrm{t}, \mathrm{J}=7.2 \mathrm{~Hz}, 3 \mathrm{H}), 2.64-2.75(\mathrm{~m}, 2 \mathrm{H}), 3.45(\mathrm{br}, 1 \mathrm{H}), 4.18$ (q, J=7.2Hz, $2 \mathrm{H}), 5.08-5.13(\mathrm{~m}, 1 \mathrm{H}), 7.26-7.32(\mathrm{~m}, 4 \mathrm{H}) ;{ }^{13} \mathrm{CNMR}\left(75.4 \mathrm{MHz}, \mathrm{CDCl}_{3}\right) \delta 14.1,43.1$, 61.0, 69.6, 127.0, 128.6, 133.4, 140.9, 172.3 .

3-Hydroxy-3-(4-Fluorophenyl)-Propionic Acid Ethyl Ester (6f) ${ }^{1} \mathrm{HNMR}(300 \mathrm{MHz}$, $\left.\mathrm{CDCl}_{3}\right) \delta 1.26(\mathrm{t}, \mathrm{J}=7.2 \mathrm{~Hz}, 3 \mathrm{H}), 2.64-2.77(\mathrm{~m}, 2 \mathrm{H}), 3.45(\mathrm{br}, 1 \mathrm{H}), 4.17$ (q, J=7.2Hz, $2 \mathrm{H})$, 5.08-5.13 (m, 1H), 7.00-7.06 (m, 2H), 7.32-7.37 (m, 2H); ${ }^{13} \mathrm{CNMR}(75.4 \mathrm{MHz}$, $\left.\mathrm{CDCl}_{3}\right) \delta 14.1,43.3,60.9,69.6,115.2,115.5,127.3,128.4,138.2,163.8,172.3$;

3-Hydroxy-3-(3-Methylphenyl)-Propionic Acid Ethyl Ester (6g) ${ }^{1} \mathrm{HNMR}(300 \mathrm{MHz}$, $\left.\mathrm{CDCl}_{3}\right) \delta 1.26(\mathrm{t}, \mathrm{J}=7.2 \mathrm{~Hz}, 3 \mathrm{H}), 2.35(\mathrm{~s}, 3 \mathrm{H}), 2.68-2.77$ (m, 2H), 3.27 (br, 1H), 4.18 (q, 7.2Hz, 2H), 5.08-5.11(m, 1H), 7.08-7.26 (m, 4H); ${ }^{13} \mathrm{CNMR}\left(75.4 \mathrm{MHz}, \mathrm{CDCl}_{3}\right) \delta 14.1$, $21.4,43.3,60.8,70.3,122.7,126.3,128.4,128.5,138.2,142.4,172.4$. 
3-Hydroxy-3-(3-Chlorophenyl)-Propionic Acid Ethyl Ester (6h) ${ }^{1} \mathrm{HNMR}(300 \mathrm{MHz}$, $\left.\mathrm{CDCl}_{3}\right) \delta 1.26(\mathrm{t}, \mathrm{J}=7.2 \mathrm{~Hz}, 3 \mathrm{H}), 2.68-2.70(\mathrm{~m}, 2 \mathrm{H}), 3.46(\mathrm{br}, 1 \mathrm{H}), 4.18(\mathrm{q}, \mathrm{J}=7.2 \mathrm{H}, 2 \mathrm{H})$, 5.09(m, 1H), 7.22-7.28 (m, 3H), $7.38(\mathrm{~s}, 1 \mathrm{H}) ;{ }^{13} \mathrm{CNMR}\left(75.4 \mathrm{MHz}, \mathrm{CDCl}_{3}\right) \delta 14.1$, $43.2,61.0,69.6,123.8,125.9,127.8,129.8,134.4,144.5,172.2$.

3-Hydroxy-3-(2-Methylphenyl)-Propionic Acid Ethyl Ester (6i) ${ }^{1} \mathrm{H}$ NMR (300 $\left.\mathrm{MHz}, \mathrm{CDCl}_{3}\right): \delta 1.29(\mathrm{t}, J=7.2 \mathrm{~Hz}, 3 \mathrm{H}), 2.35(\mathrm{~s}, 3 \mathrm{H}), 2.62-2.75(\mathrm{~m}, 2 \mathrm{H}), 3.27$ (br, 1H), $4.20(\mathrm{q}, J=7.2 \mathrm{~Hz}, 2 \mathrm{H}), 5.34-5.38(\mathrm{~m}, 1 \mathrm{H}), 7.13-7.26(\mathrm{~m}, 3 \mathrm{H}), 7.49-7.52(\mathrm{~m}$, 1H). ${ }^{13} \mathrm{C} \mathrm{NMR}\left(75.4 \mathrm{MHz}, \mathrm{CDCl}_{3}\right): \delta 14.1,18.9,42.1,60.9,68.9,125.2,126.3,127.5$, $130.4,134.2,140.4,172.6$.

3-Hydroxy-Butyric Acid Ethyl Ester (8a) ${ }^{1} \mathrm{H}$ NMR $(300 \mathrm{MHz}, \mathrm{CDCl} 3)$ : $\delta 1.24$ (d, J $=6.6 \mathrm{~Hz}, 3 \mathrm{H}), 1.29(\mathrm{t}, \mathrm{J}=7.2 \mathrm{~Hz}, 3 \mathrm{H}), 2.43-2.53(\mathrm{~m}, 2 \mathrm{H}), 3.07$ (br, 1H), 4.19 (q, J = $7.2 \mathrm{~Hz}, 2 \mathrm{H}) .{ }^{13} \mathrm{C} \mathrm{NMR}(75.4 \mathrm{MHz}, \mathrm{CDCl} 3): \delta 14.1,22.4,42.7,60.6,64.2,172.9$.

3-Hydroxy-Butyric Acid Isopropyl Ester (8b) ${ }^{1} \mathrm{H}$ NMR (300 MHz, CDCl3): $\delta 1.23$ $(\mathrm{d}, \mathrm{J}=6.3 \mathrm{~Hz}, 3 \mathrm{H}), 1.26(\mathrm{~d}, \mathrm{~J}=6.3 \mathrm{~Hz}, 6 \mathrm{H}), 2.31-2.46(\mathrm{~m}, 2 \mathrm{H}), 3.28(\mathrm{br}, 1 \mathrm{H})$, 4.12-4.18 (m, 1H), 4.97-5.05 (m, 1H). ${ }^{13} \mathrm{C}$ NMR (75.4 MHz, CDCl3): $\delta$ 21.6, 22.2, 42.9, 64.1, 68.0, 172.3 .

3-Hydroxy-Butyric Acid Tert-butyl Ester (8c) ${ }^{1} \mathrm{H}$ NMR (300 MHz, CDCl3): $\delta 1.19$ $(\mathrm{d}, \mathrm{J}=6.3 \mathrm{~Hz}, 3 \mathrm{H}), 1.45(\mathrm{~s}, 9 \mathrm{H}), 2.32-2.38(\mathrm{~m}, 2 \mathrm{H}), 3.23$ (br, 1H), 4.08-4.20 (m, 1H). ${ }^{13} \mathrm{C}$ NMR (75.4 MHz, CDCl3): $\delta$ 22.2, 28.1, 43.7, 64.3, 81.2, 171.4.

3-Hydroxy-4,4-Dimethyl-Pentanoic Acid Ethyl Ester (8d) ${ }^{1} \mathrm{H}$ NMR (300MHz, CDCl3): $\delta 0.93(\mathrm{~s}, 9 \mathrm{H}), 1.29(\mathrm{t}, \mathrm{J}=7.2 \mathrm{~Hz}, 3 \mathrm{H}), 2.31-2.56(\mathrm{~m}, 2 \mathrm{H}), 2.92(\mathrm{~d}, \mathrm{~J}=3.6$ $\mathrm{Hz}, 3 \mathrm{H}), 3.71(\mathrm{~m}, 1 \mathrm{H}), 4.18$ (q, J = 7.2 Hz, 2H). ${ }^{13} \mathrm{C} \mathrm{NMR}(75.4 \mathrm{MHz}, \mathrm{CDCl} 3): \delta 14.1$, $25.5,34.3,36.5,60.7,75.4,173.9$.

4-Chloro-3-Hydroxy-Butyric Acid Ethyl Ester (8e) ${ }^{1} \mathrm{H}$ NMR (300 $\left.\mathrm{MHz}, \mathrm{CDCl}_{3}\right)$ : $\delta$ $1.28(\mathrm{t}, J=7.2 \mathrm{~Hz}, 3 \mathrm{H}), 2.55-2.59(\mathrm{~m}, 2 \mathrm{H}), 3.47(\mathrm{br}, 1 \mathrm{H}), 3.55(\mathrm{~d}, J=5.1 \mathrm{~Hz}, 2 \mathrm{H})$, $4.12(\mathrm{q}, J=7.2 \mathrm{~Hz}, 2 \mathrm{H}), 4.22-4.31(\mathrm{~m}, 1 \mathrm{H}) .{ }^{13} \mathrm{C} \mathrm{NMR}\left(75.4 \mathrm{MHz}, \mathrm{CDCl}_{3}\right): \delta 13.9$, $38.4,48.0,60.8,67.7,171.6$. 


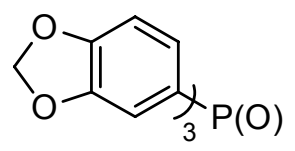

2

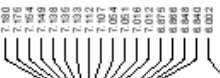

vilf

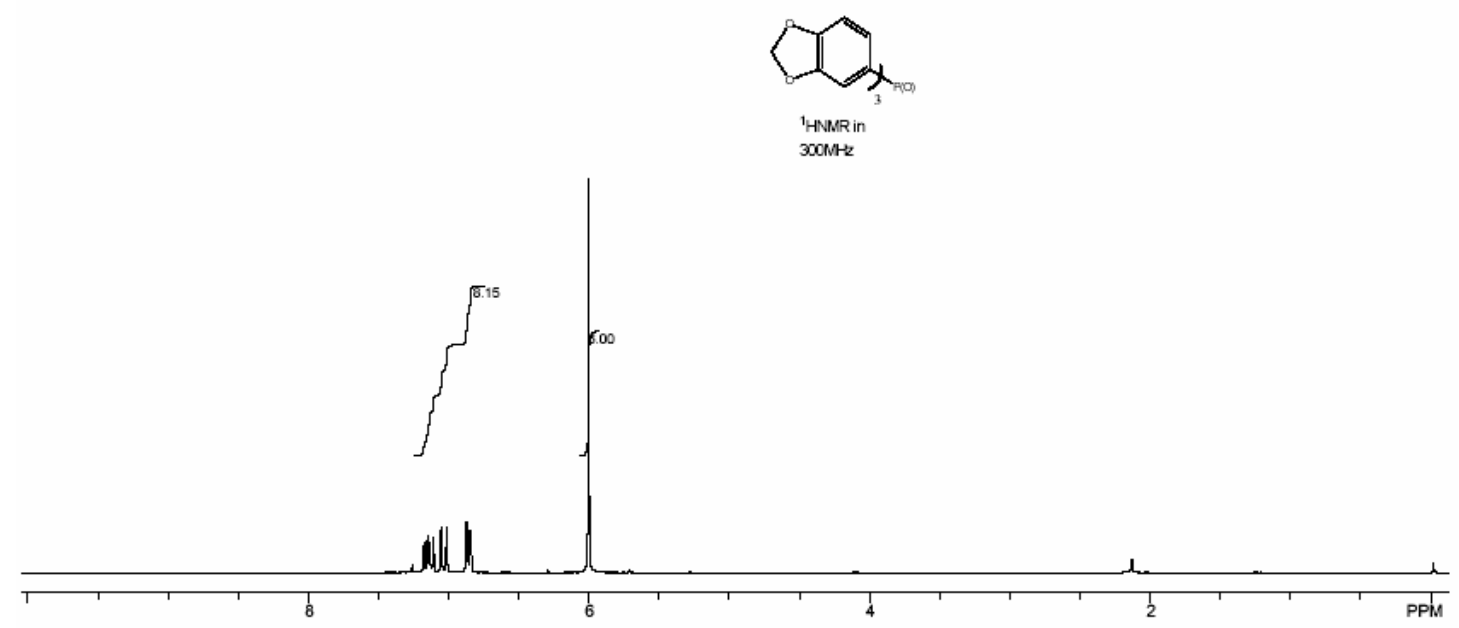

$\mathbb{N}_{3}$

${ }^{31}$ PNMR in

$300 \mathrm{MHz}$

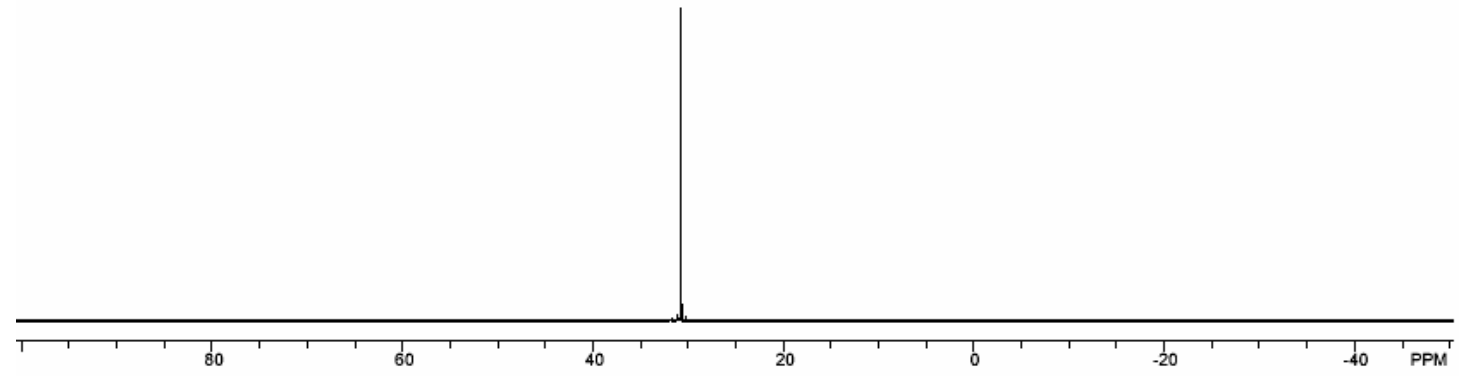




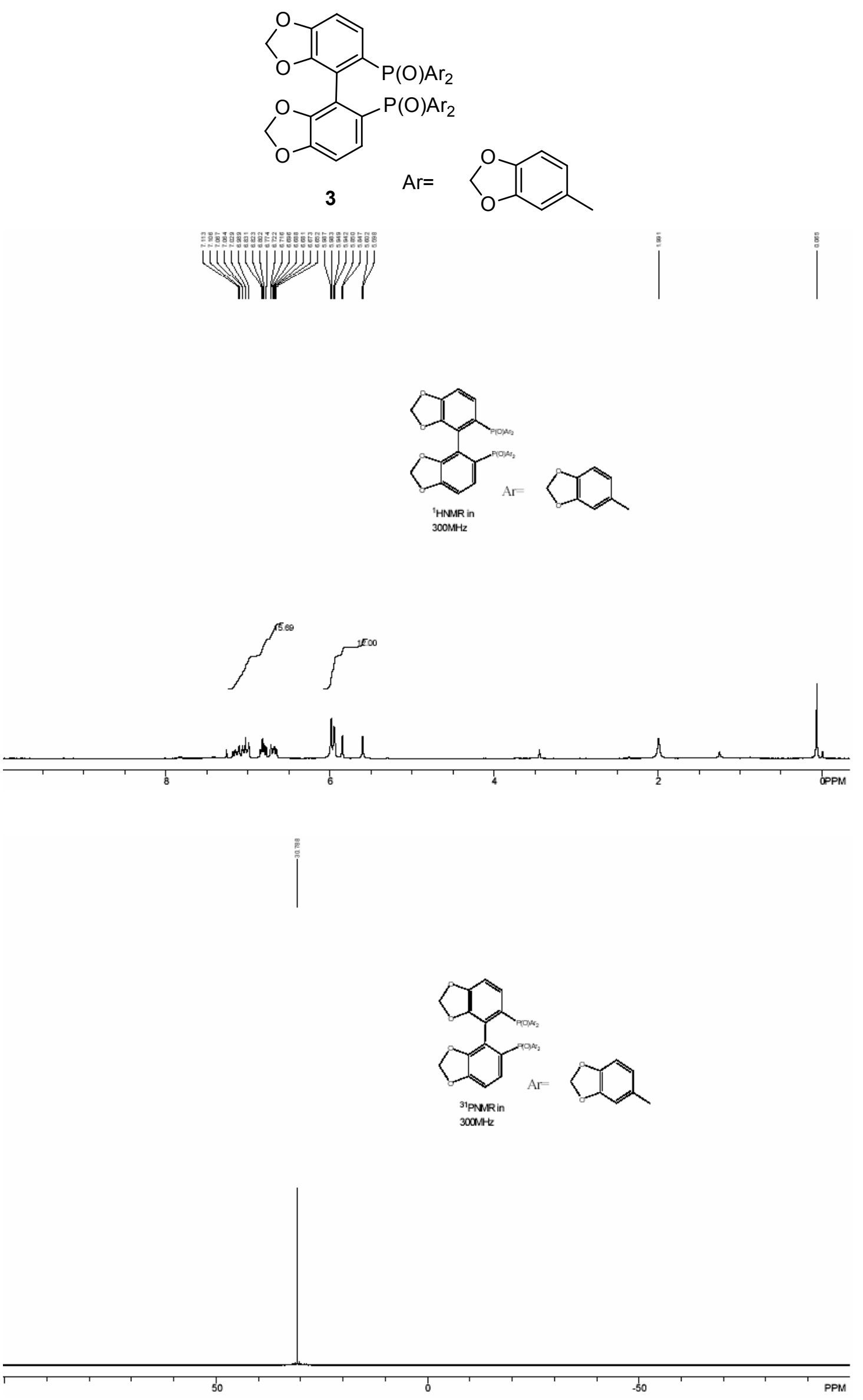


<smiles>Cc1ccc2c(c1)OCO2</smiles>
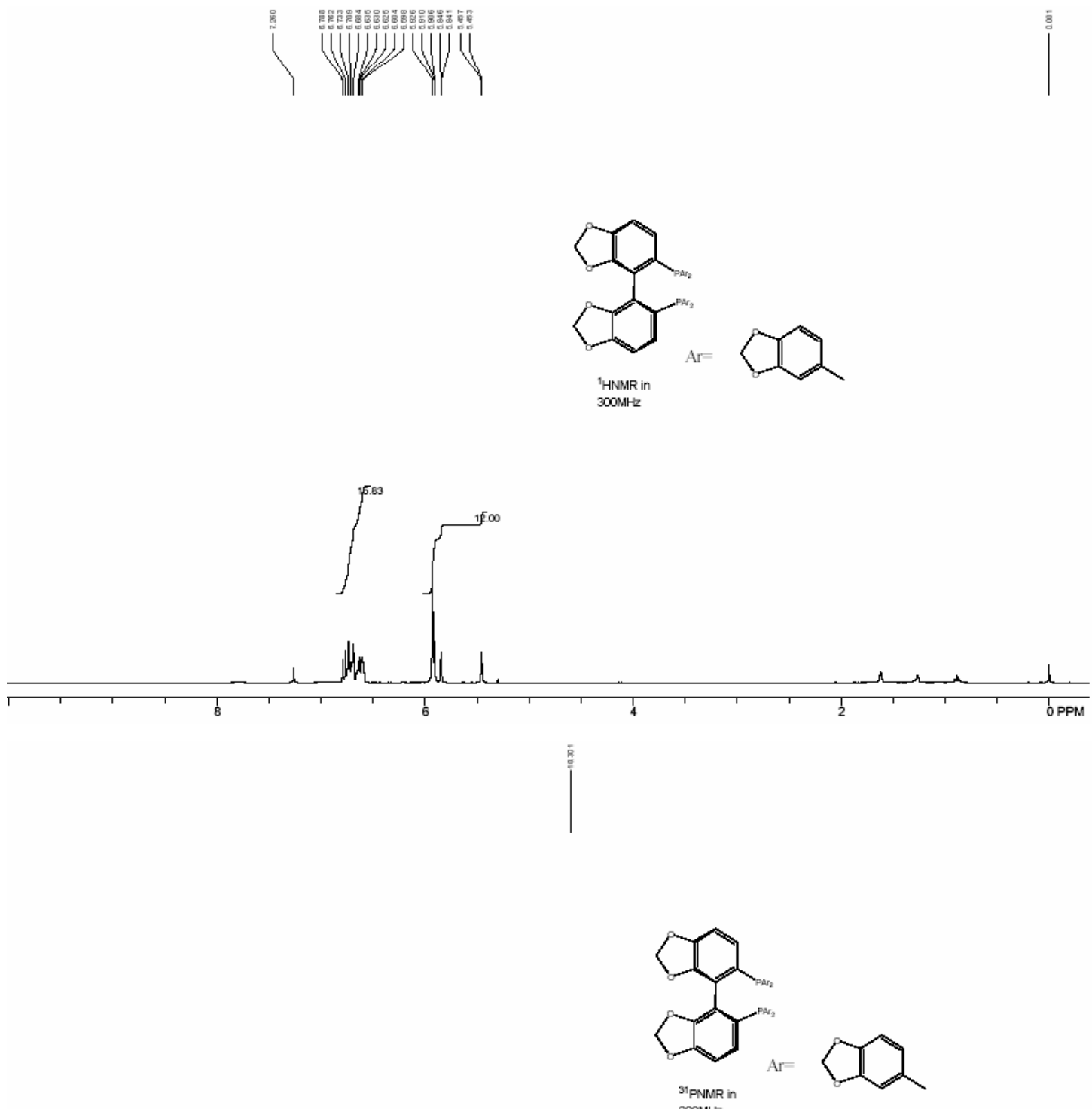

$300 \mathrm{MHz}$

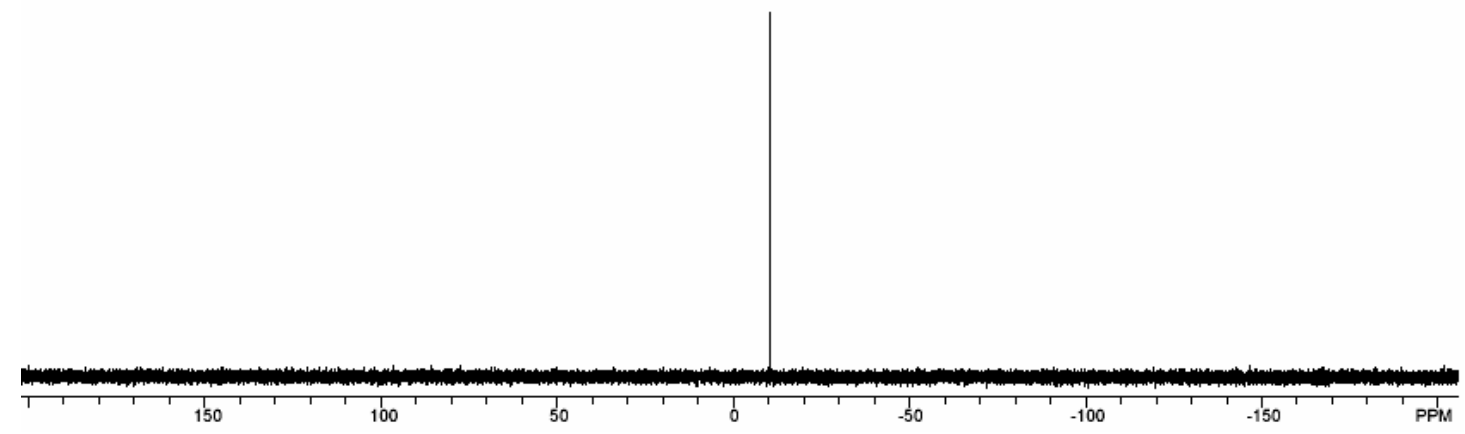

S7 
<smiles>CCOC(=O)CC(O)c1ccccc1</smiles>

$6 a$

(U)<smiles>CCOC(=O)CC(O)c1ccccc1</smiles>

'HNMR in

$300 \mathrm{MHz}$

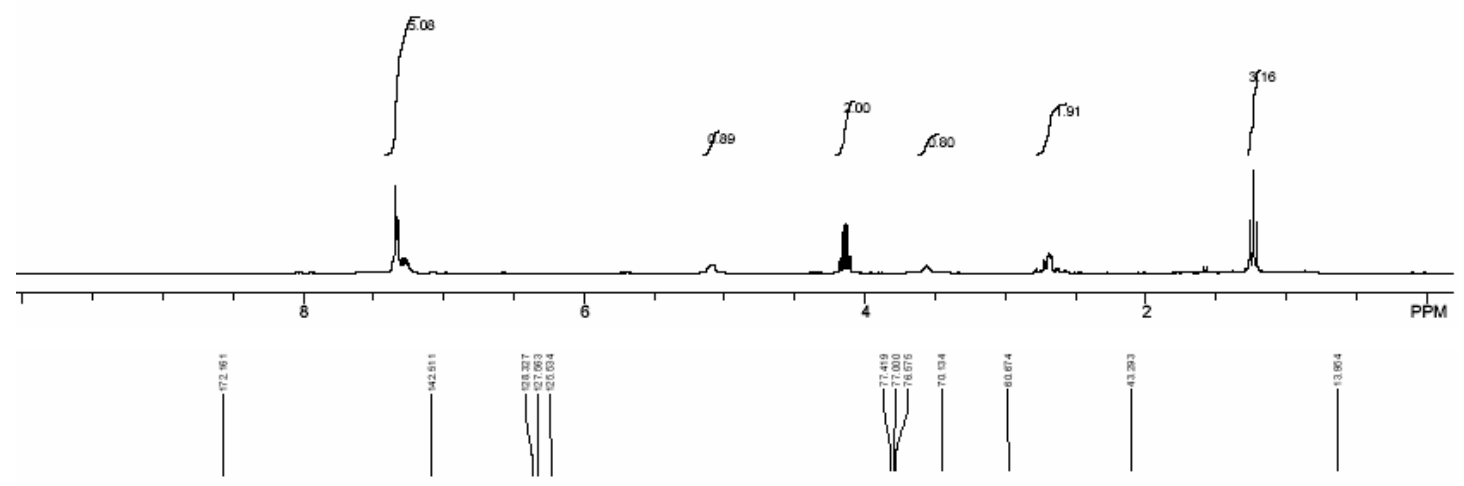

$\overbrace{}^{\mathrm{OH}}$

${ }^{13} \mathrm{CNMR}$ in

$300 \mathrm{MHz}$

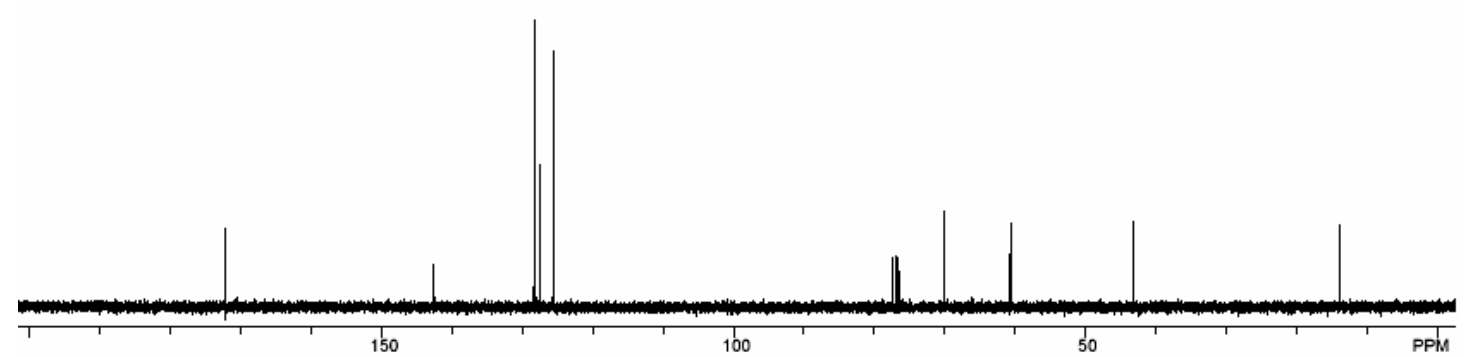


<smiles>CCOC(=O)CC(O)c1ccc(OC)cc1</smiles>

$6 b$
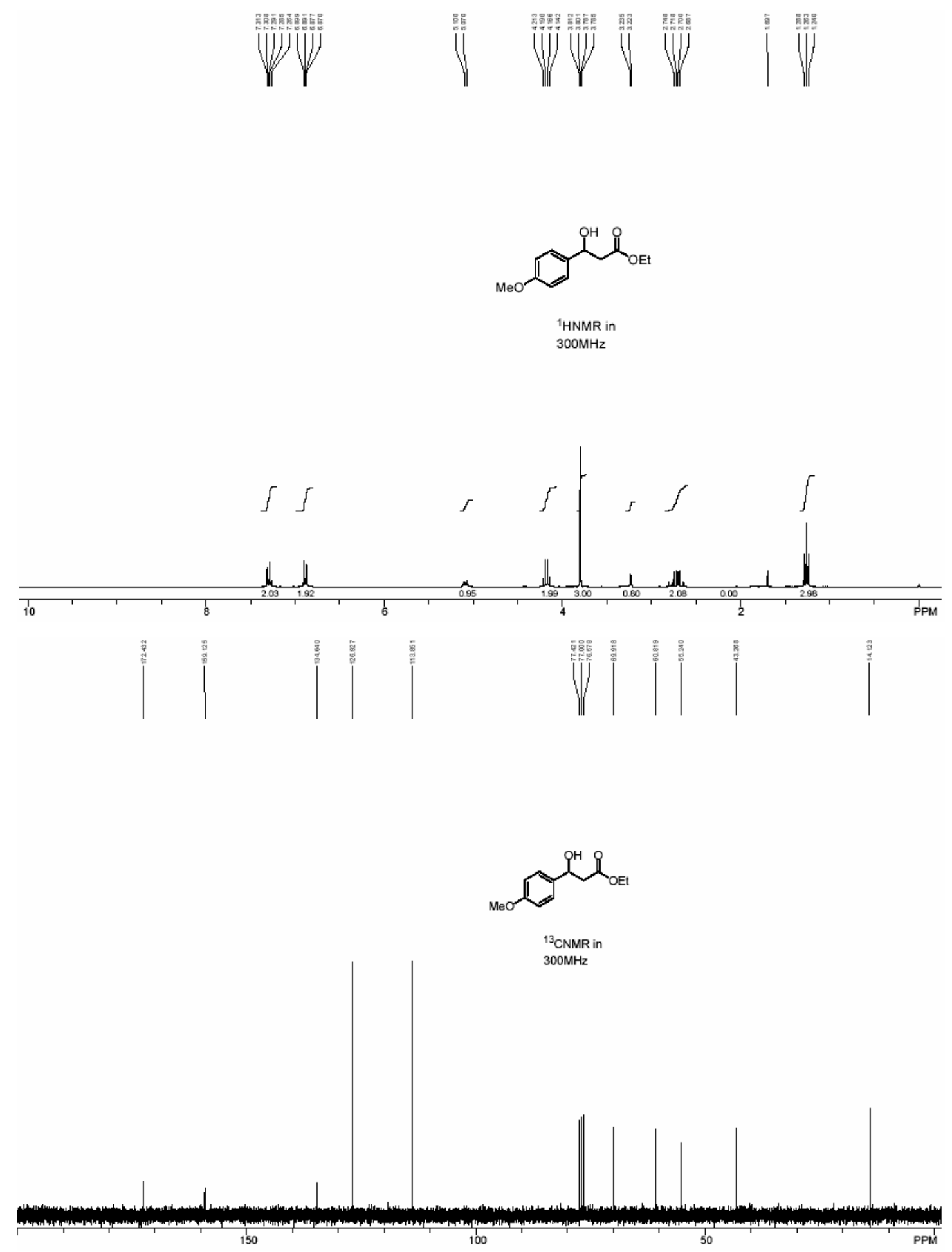
<smiles>CCOC(=O)CC(O)c1ccc(C)cc1</smiles>

$6 c$

V(l)

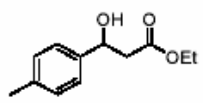

${ }^{1} \mathrm{HNMR}$ in
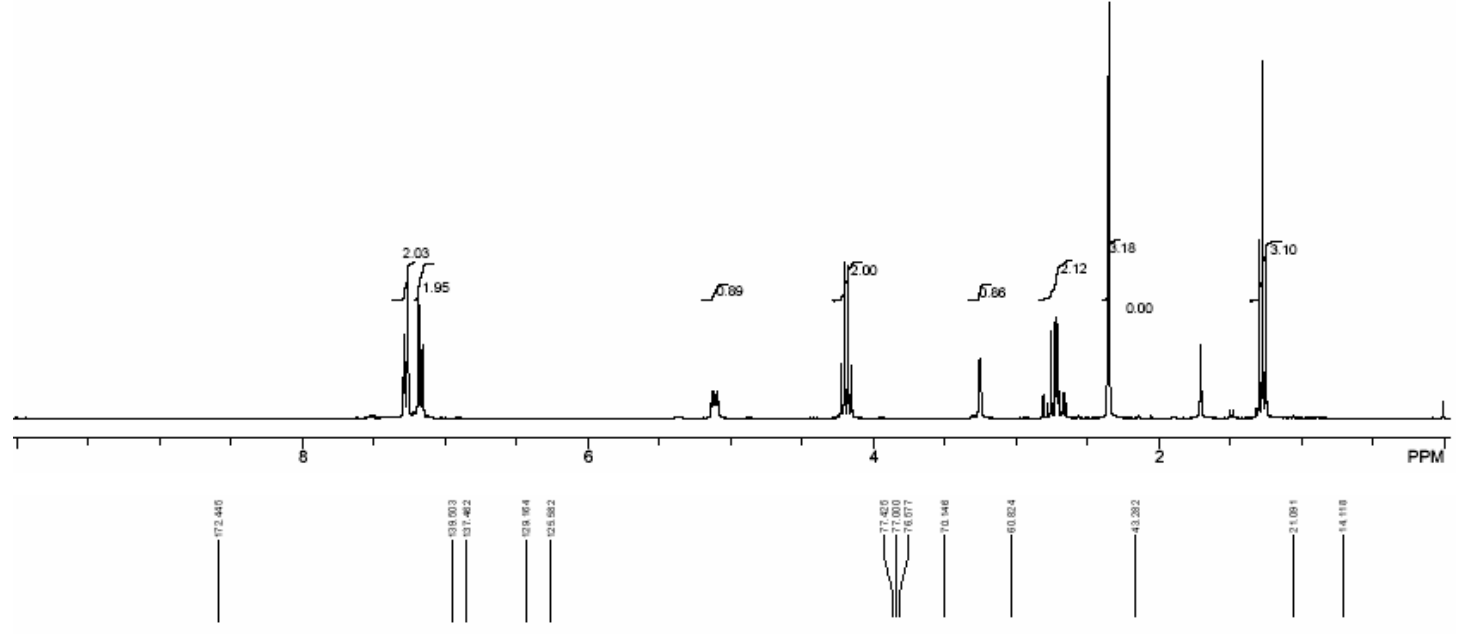

11

${ }^{13}$ CNMR in $300 \mathrm{MHz}$

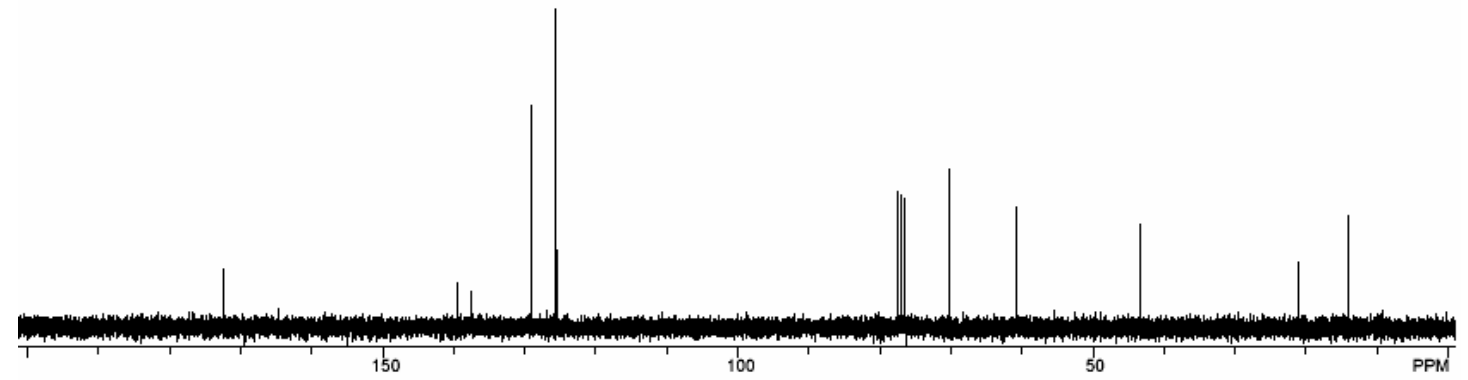


<smiles>CCOC(=O)CC(O)c1ccc(Br)cc1</smiles>

$6 d$

||

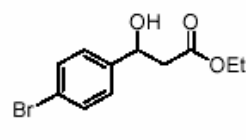

${ }^{1} \mathrm{HNMR}$ in

$300 \mathrm{MHz}$
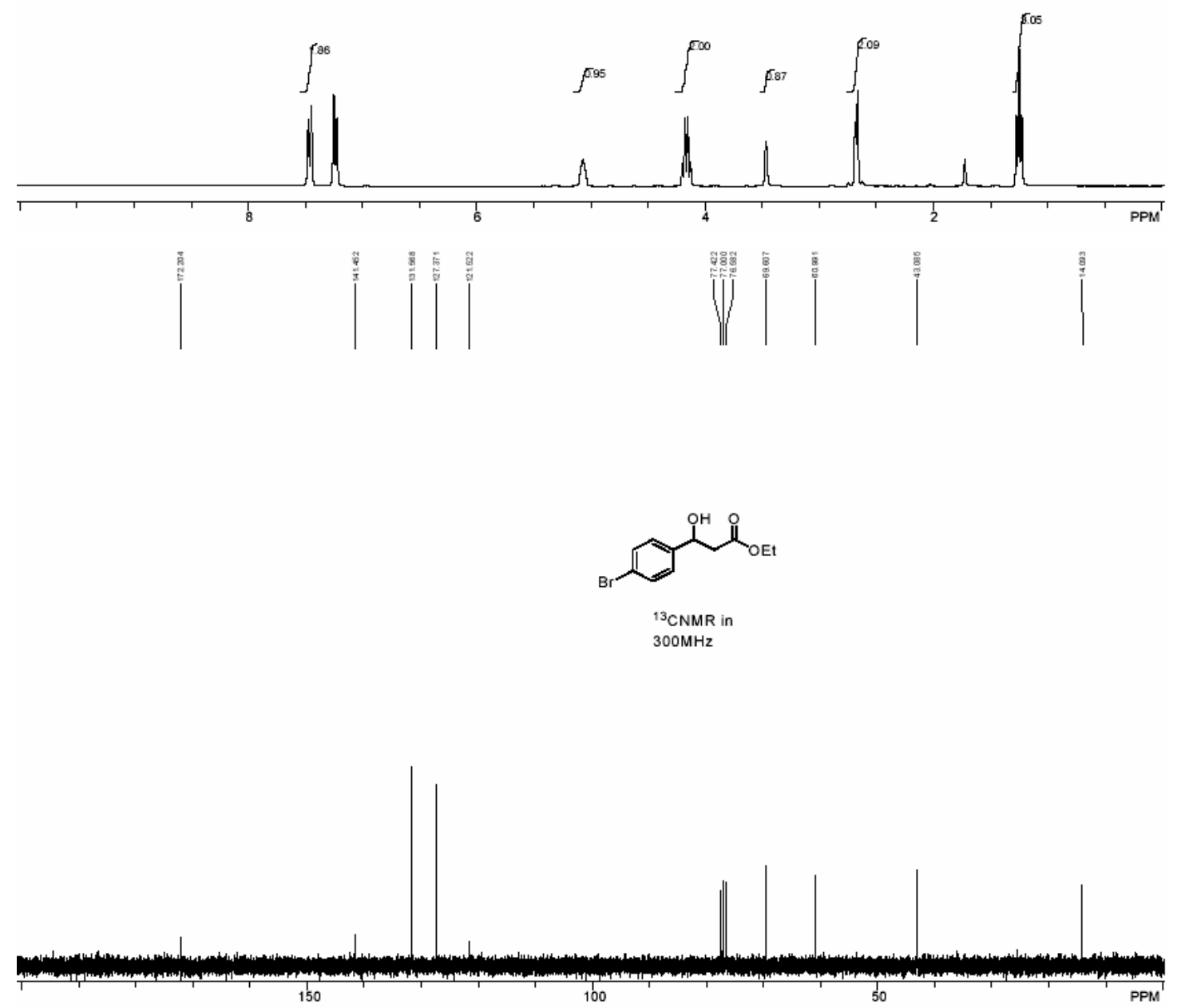
<smiles>CCOC(=O)CC(O)c1ccc(Cl)cc1</smiles>

$6 e$
F
(4)
บ

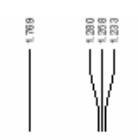

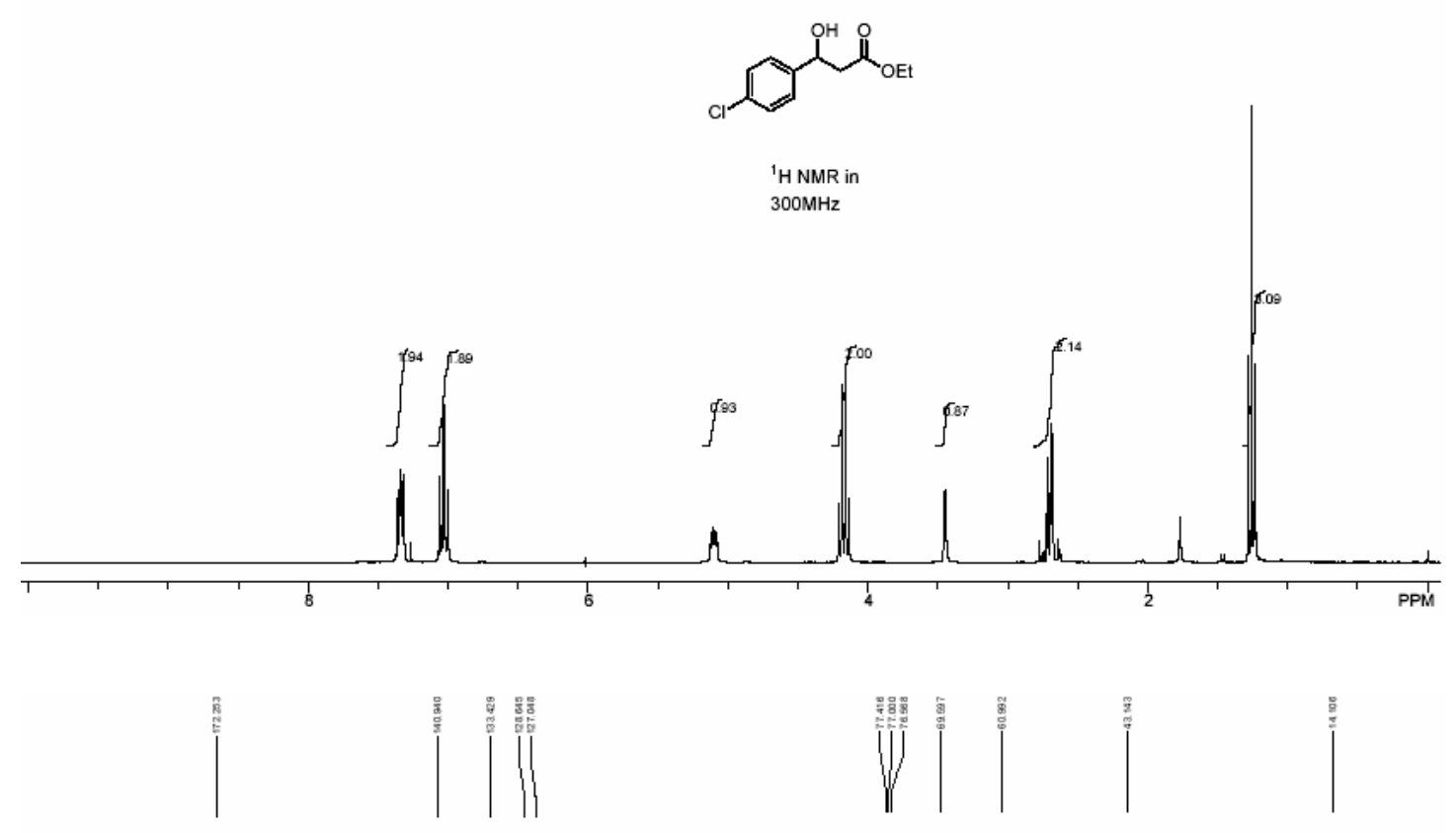

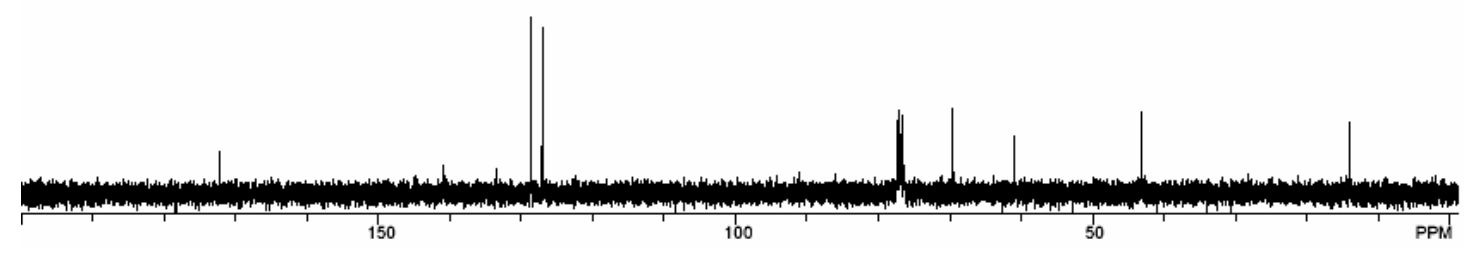


<smiles>CCOC(=O)CC(O)c1ccc(F)cc1</smiles>

\section{$6 f$}

W
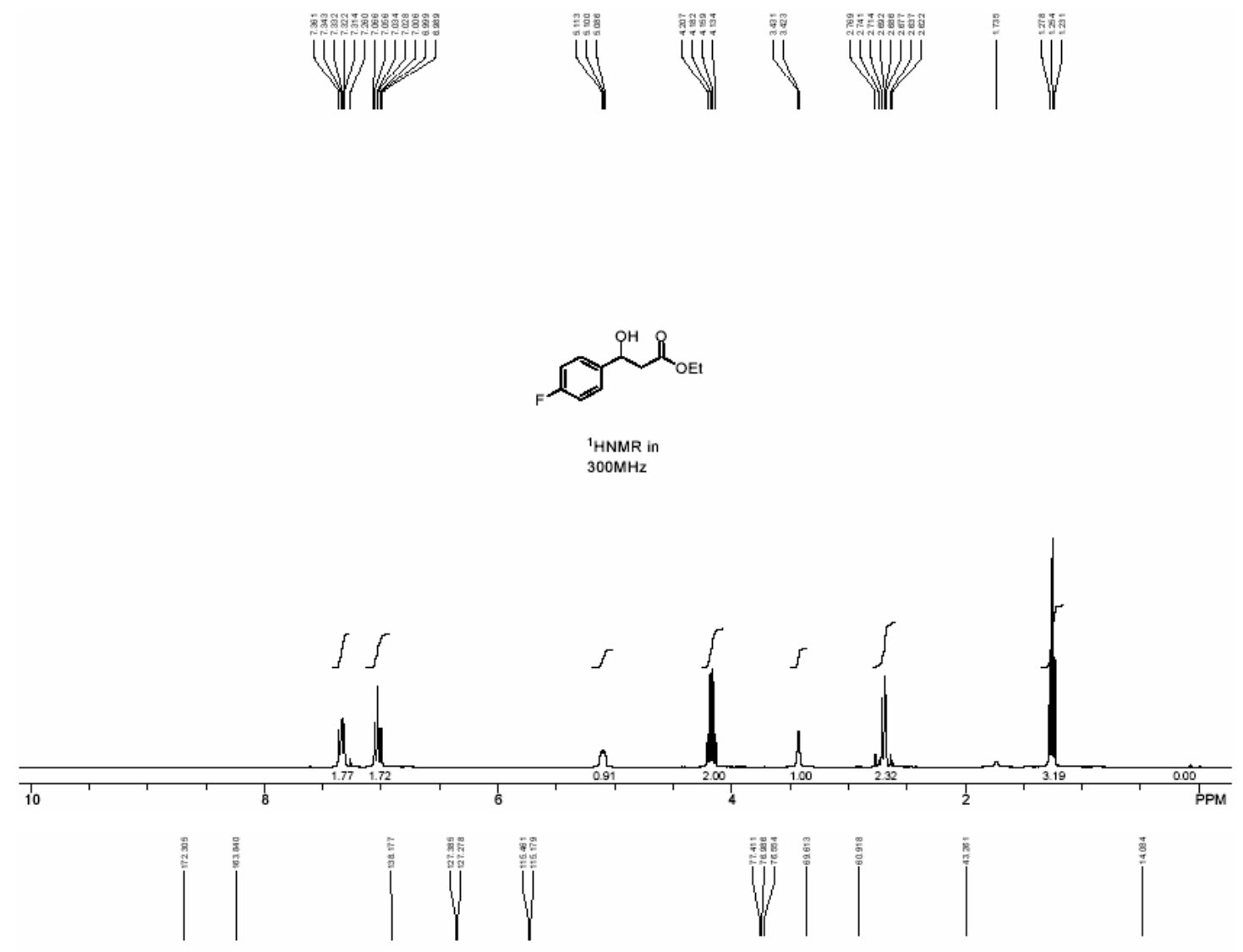

皮

${ }^{13} \mathrm{CNMR}$ in

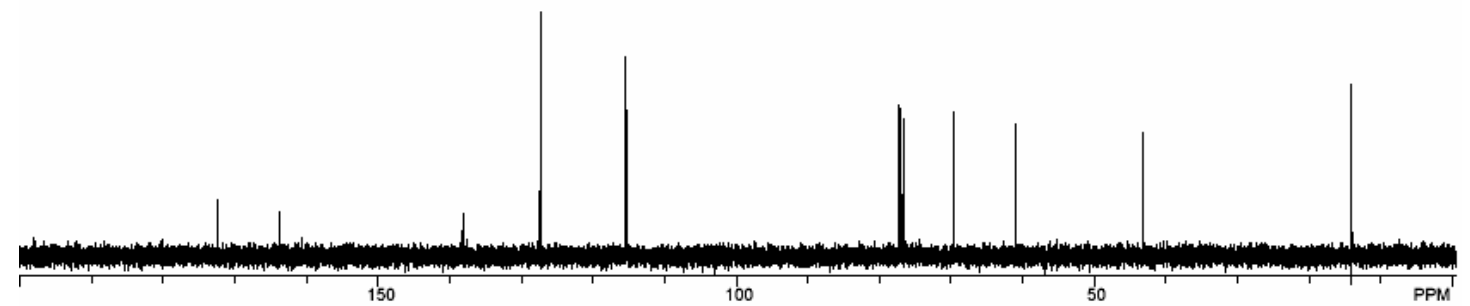


<smiles>CCOC(=O)CC(O)c1cccc(C)c1</smiles>

$6 \mathrm{~g}$

(IIJ)

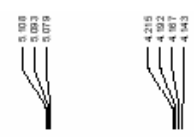

|<smiles>CCOC(=O)CC(O)c1cccc(C)c1</smiles>
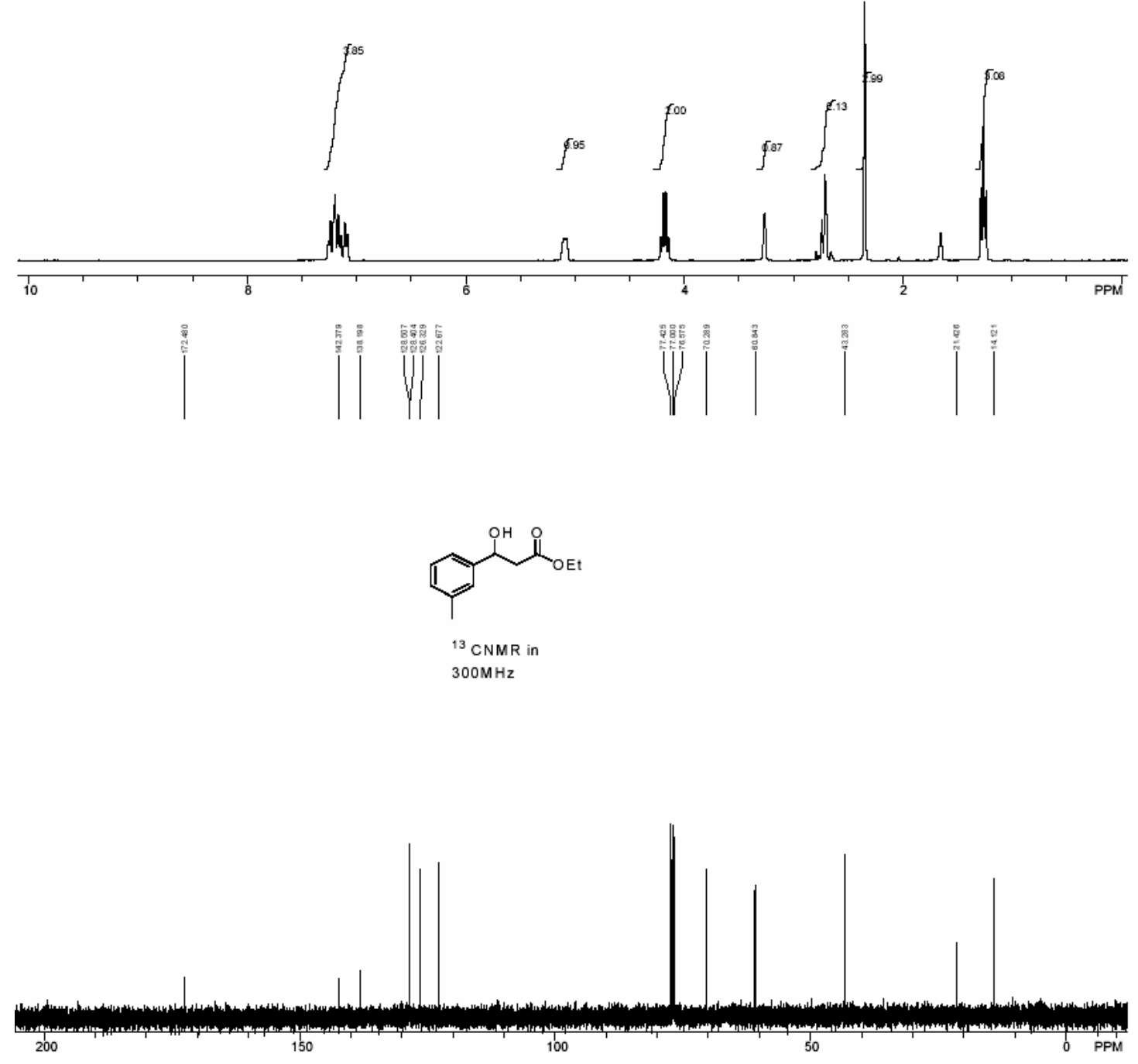

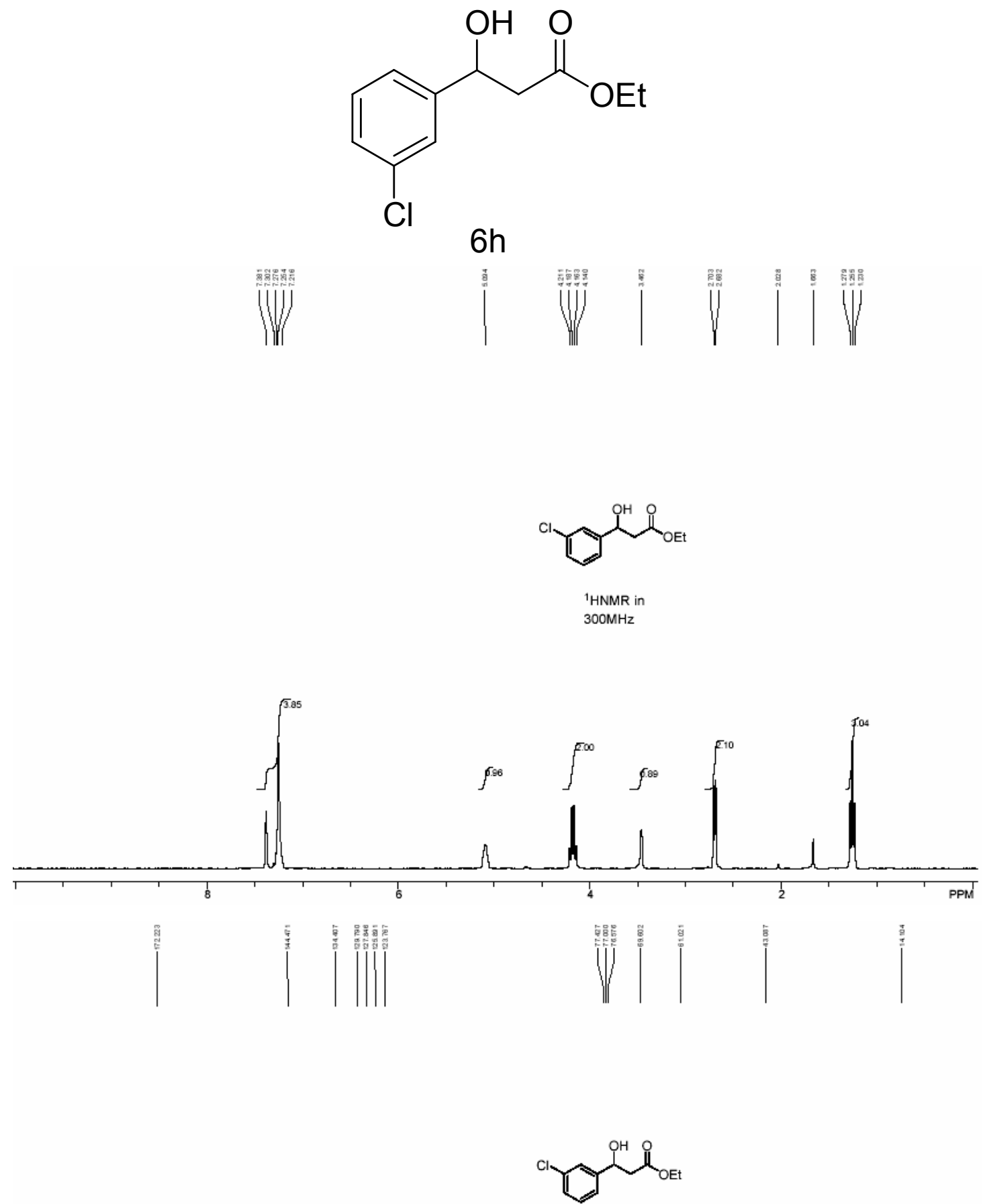

${ }^{13} \mathrm{CNMR}$ in

$300 \mathrm{MHz}$

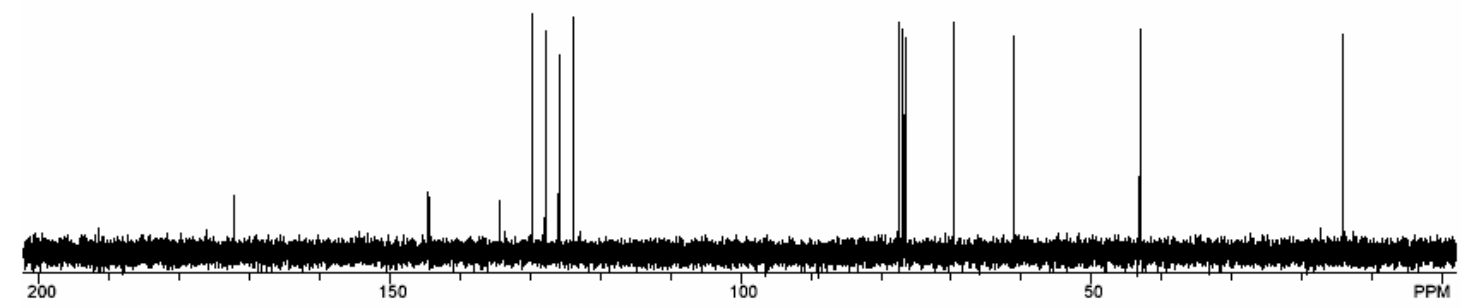


<smiles>CCOC(=O)CC(O)c1ccccc1C</smiles>

$6 i$

U

แ

Џบ

|UI)

ไ1.

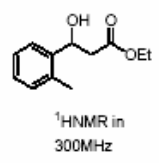
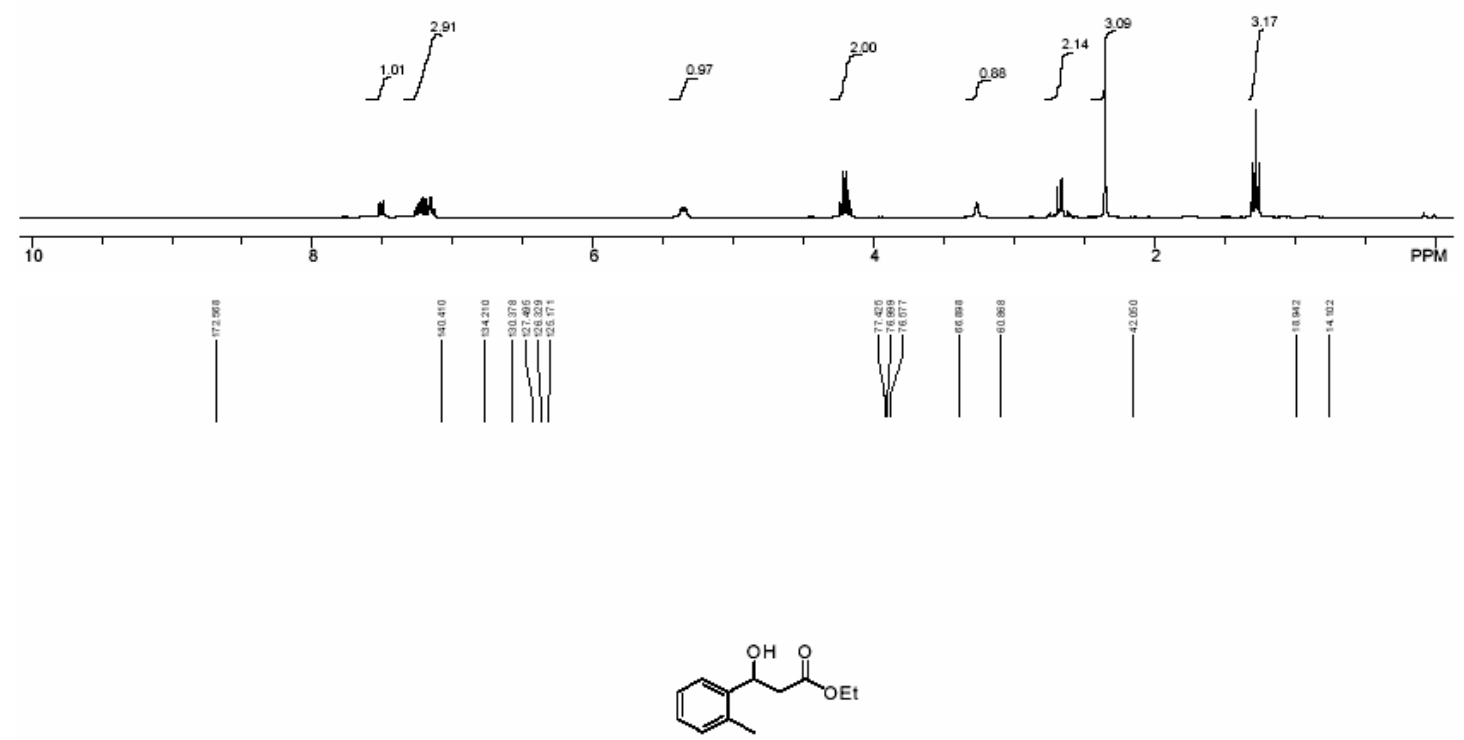

${ }^{13} \mathrm{CNMR}$ in

$300 \mathrm{MHz}$

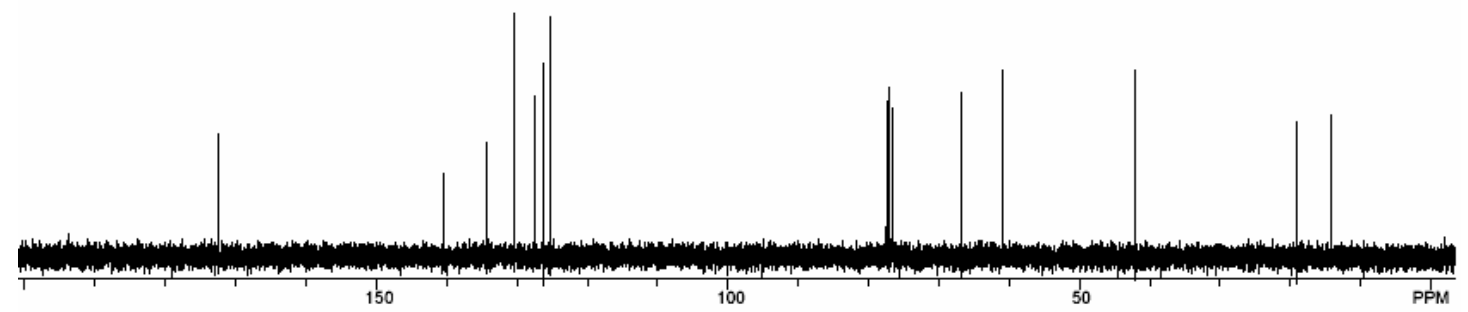


<smiles>CCOC(=O)CC(C)O</smiles>

\section{$8 a$}

앤 유

${ }^{1}$ HNMR in

$300 \mathrm{MHz}$

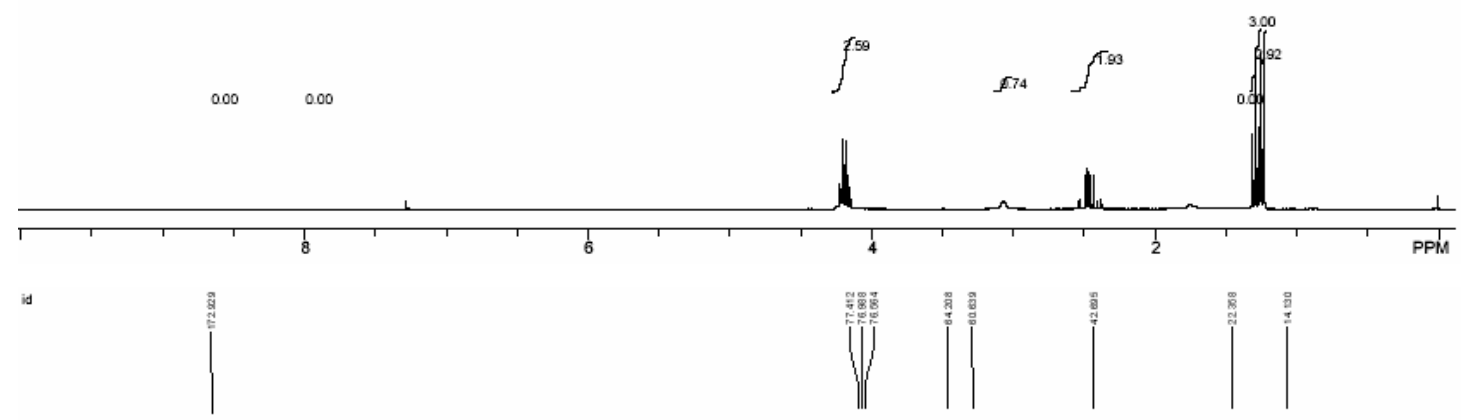

앳요요

${ }^{13} \mathrm{CNMR}$ in

300M H 
<smiles>CC(O)CC(=O)OC(C)C</smiles>

$8 b$

UW

앳이사

${ }^{1} \mathrm{HNMR}$ in

$300 \mathrm{MHz}$

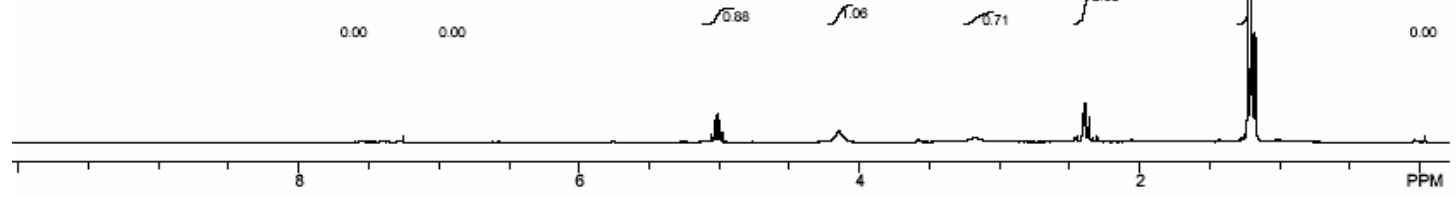

7-23-1e.fid

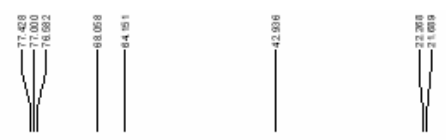

요슈.상

${ }^{13} \mathrm{CNMR}$ in

$300 \mathrm{MHz}$ 
<smiles>CC(O)CC(=O)OC(C)(C)C</smiles>

$8 c$

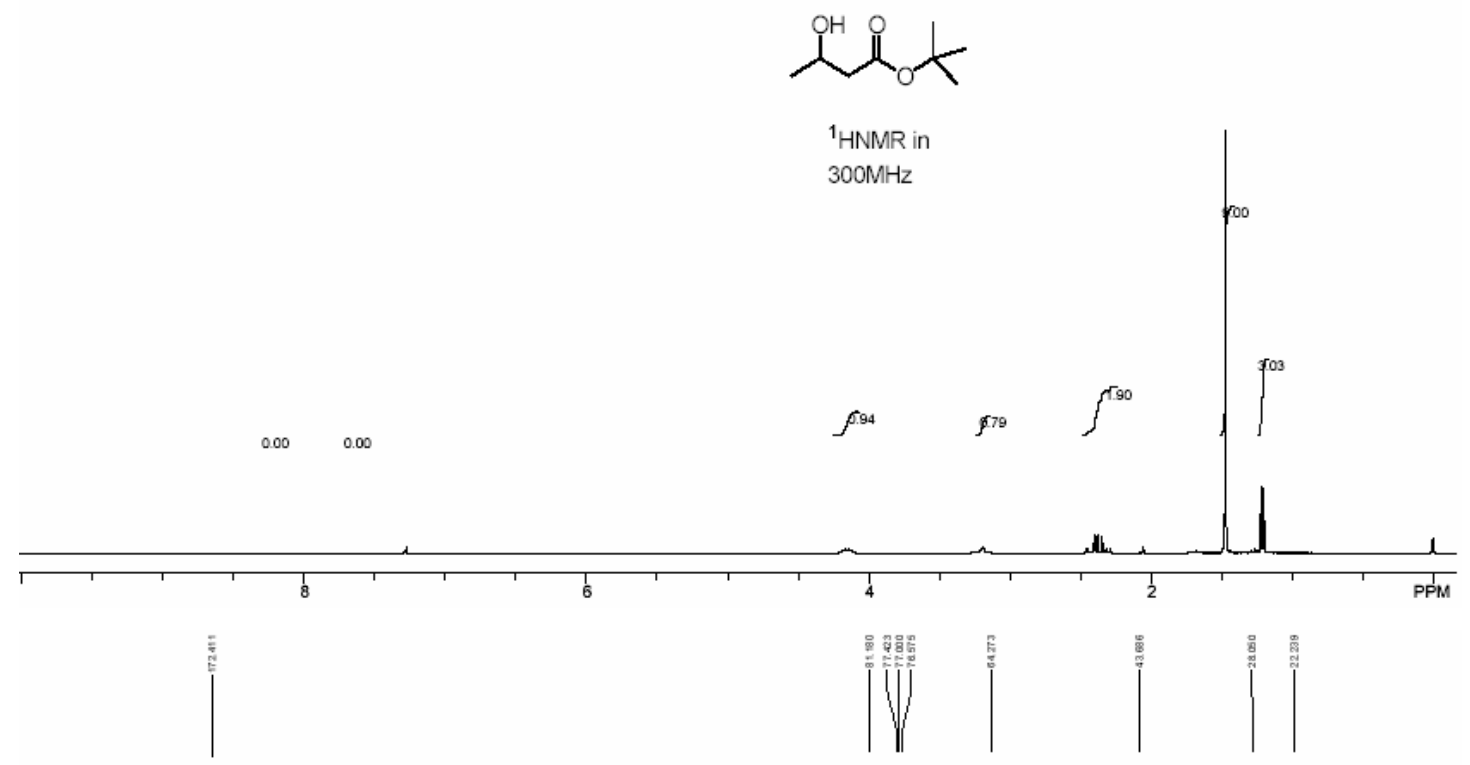

서샂

${ }^{13} \mathrm{CNMR}$ in

$300 \mathrm{MHz}$
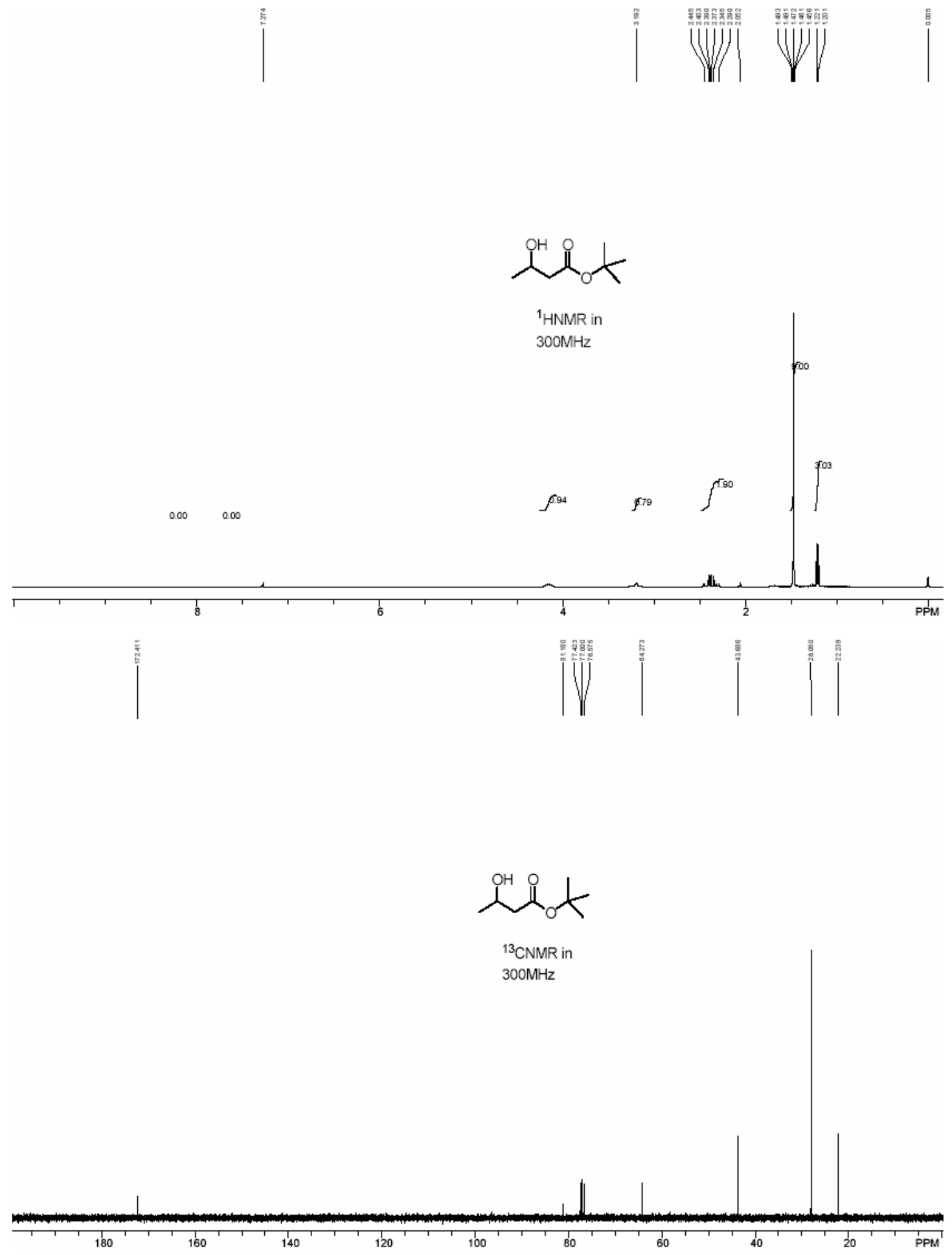
<smiles>CCOC(=O)CC(O)C(C)(C)C</smiles>

$8 d$

\section{1}

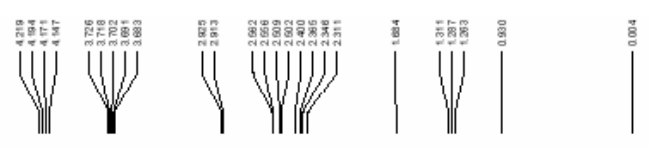
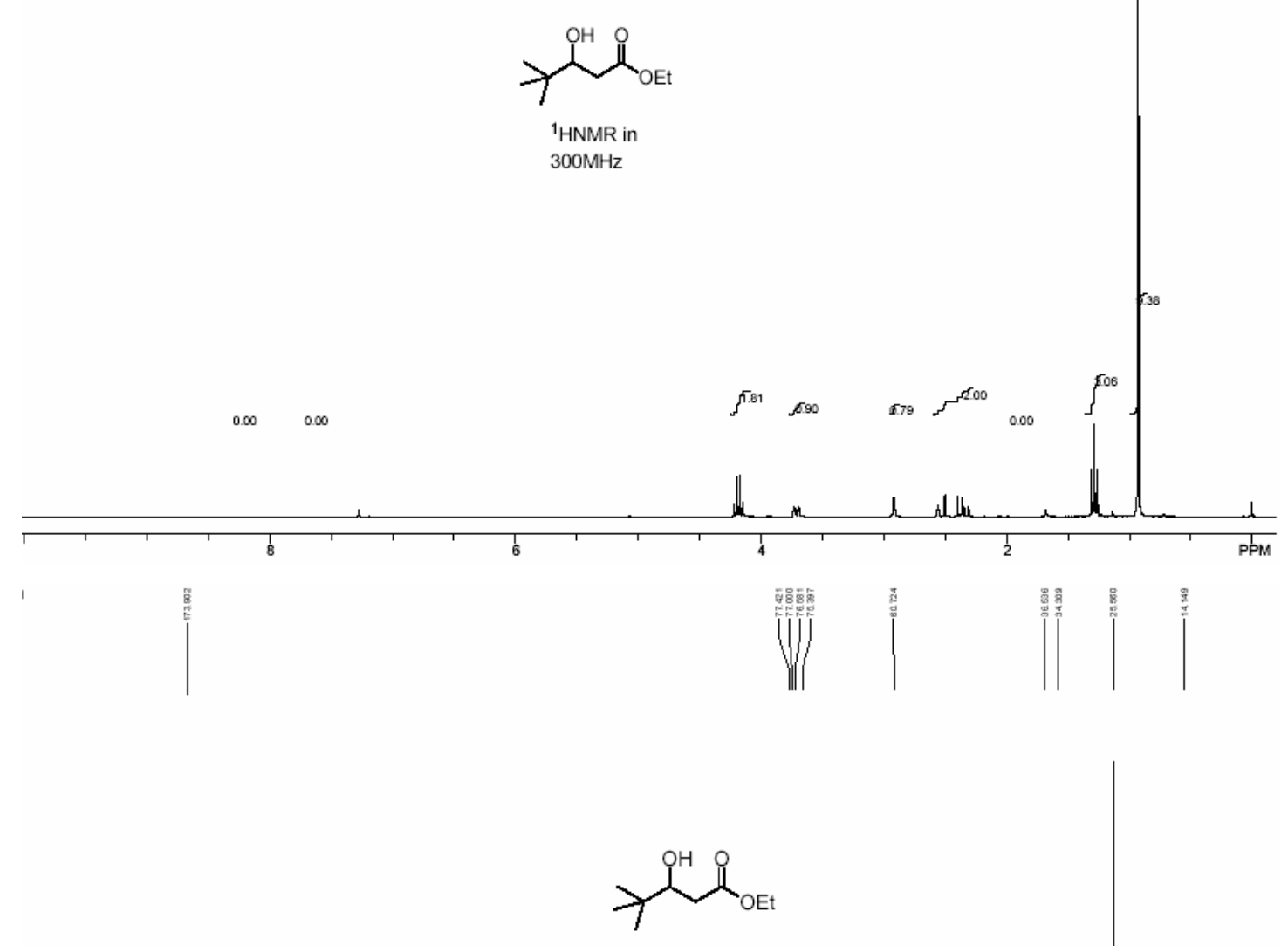

${ }^{13} \mathrm{CNMR}$ in

$300 \mathrm{MHz}$

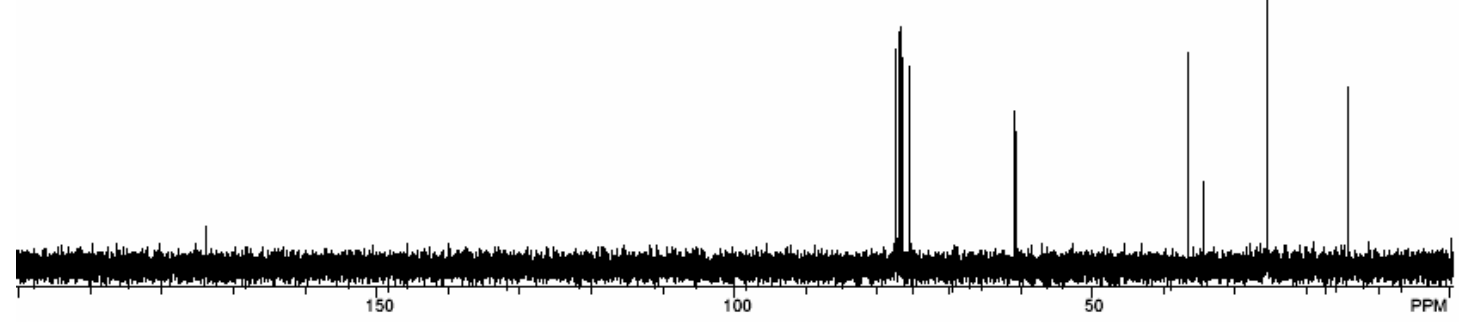


<smiles>CCOC(=O)CC(O)CCl</smiles>

\section{$8 e$}<smiles>c1cc[14c]cc1</smiles><smiles>CCOC(=O)CC(O)CCl</smiles>

${ }^{1} \mathrm{HNMR}$ in

$300 \mathrm{MHz}$
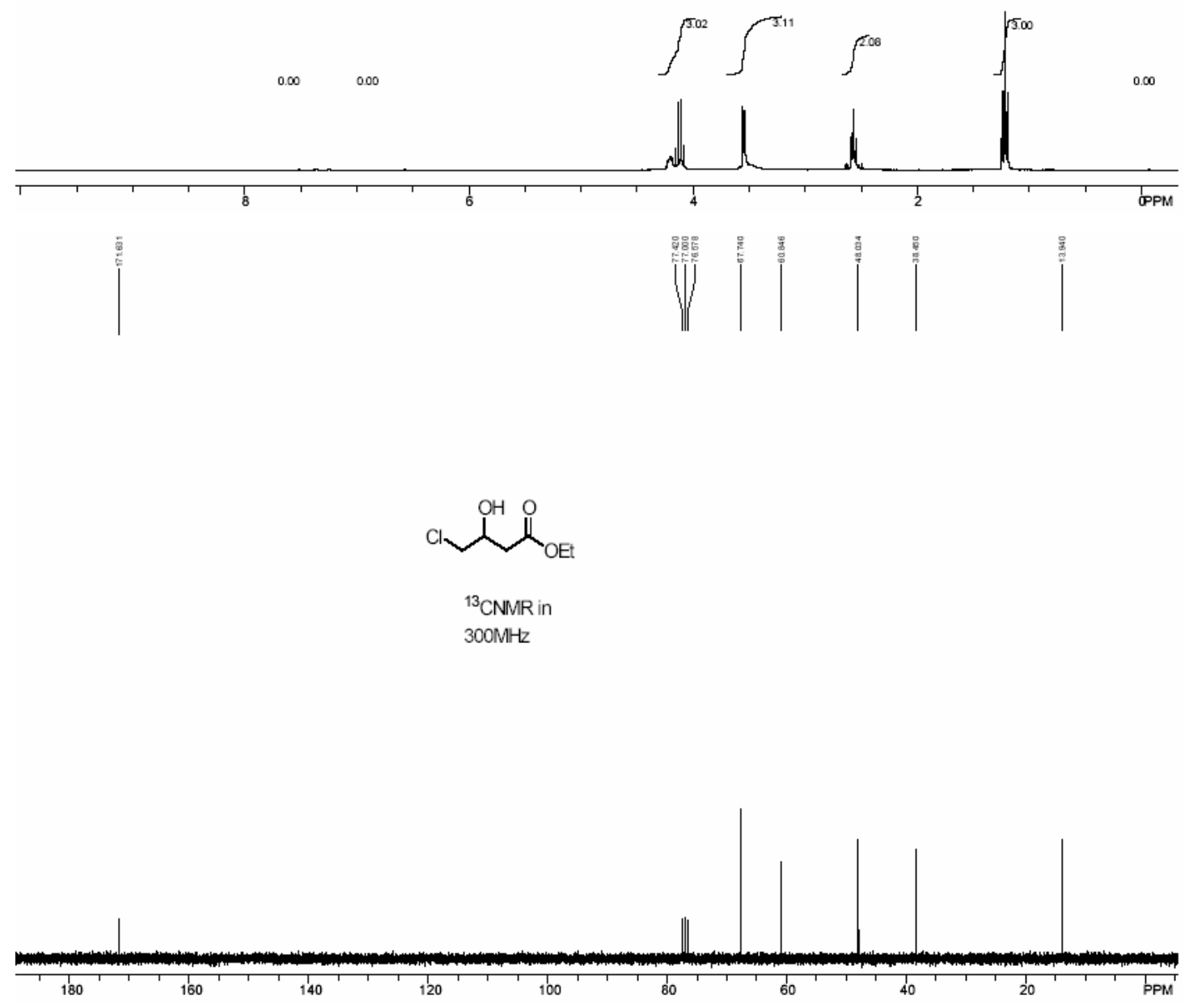


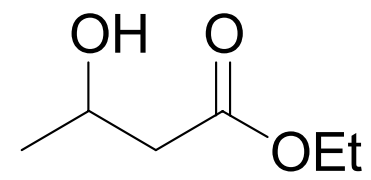

8 a racemate

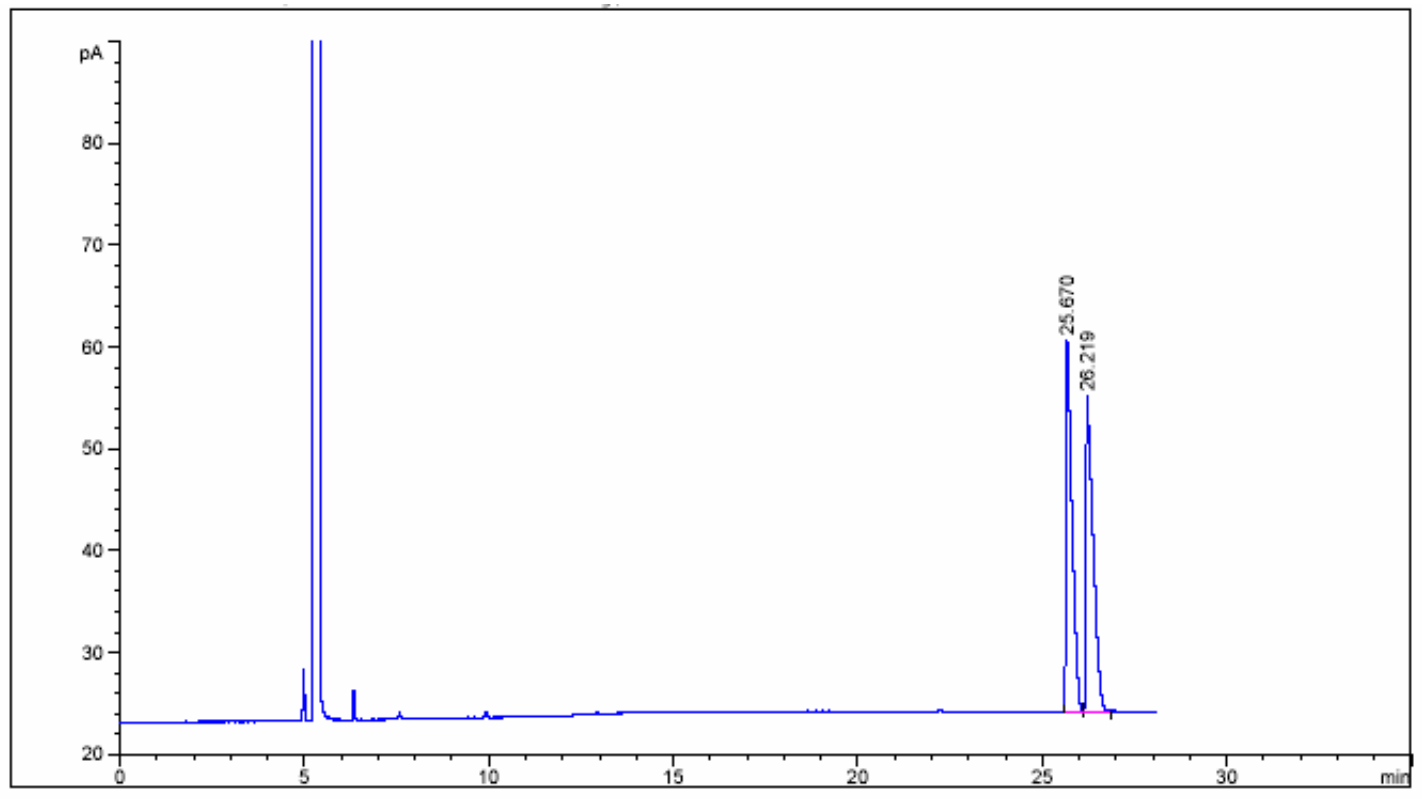

Area Percent Report

$\begin{array}{llll}\text { Sorted By } & : & \text { Signal } & \\ \text { Multiplier } & : & 1.0000 \\ \text { Dilution } & : & 1.0000 \\ \text { Sample Amount } & : & 1.00000 \quad[\mathrm{ng} / \mathrm{ul}] \quad \text { (not used in calc.) }\end{array}$

Signa1 1: FID1 A,

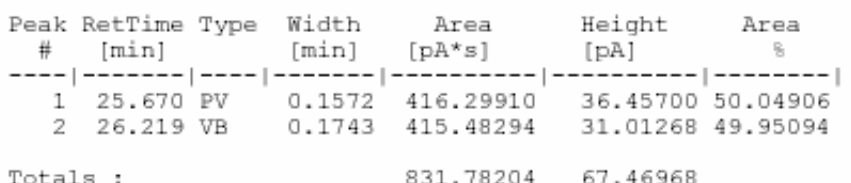

Totals :

$831.78204 \quad 67.46968$

Results obtained with enhanced integrator!

$\star * *$ End of Report $* * *$ 


\section{Table 1 entry1}

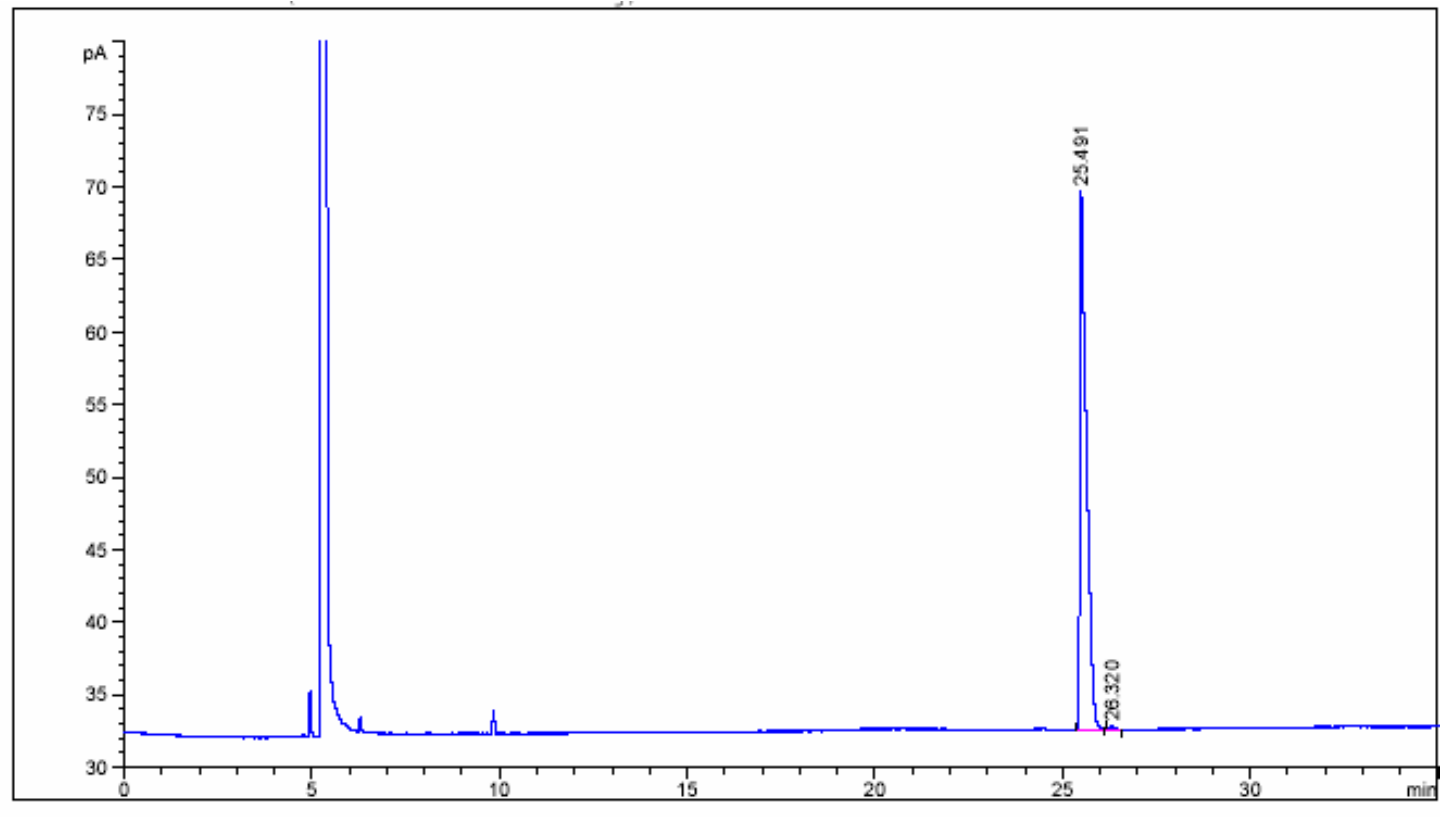

Area Percent Report

\begin{tabular}{|c|c|c|c|c|}
\hline Sorted By & : & Signal & & \\
\hline Multiplier & : & 1.0000 & & \\
\hline Dilution & : & 1.0000 & & \\
\hline Sample Amount & $:$ & 1.00000 & {$[\mathrm{ng} / \mathrm{ul}]$} & (not used in calc.) \\
\hline \multicolumn{5}{|l|}{ Signal 1: FID1 A, } \\
\hline $\begin{array}{l}\text { Peak RetTime Type } \\
\# \quad[\text { min] }\end{array}$ & $\begin{array}{l}\text { Width } \\
\text { [min] }\end{array}$ & $\begin{array}{r}\text { Area } \\
{\left[\mathrm{pA}^{*} \mathrm{~s}\right]}\end{array}$ & $\begin{array}{l}\text { Height } \\
{[\mathrm{pA}]}\end{array}$ & $\begin{array}{c}\text { Area } \\
\frac{8}{8}\end{array}$ \\
\hline 125.491 PB & 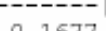 & 45709436 & - & 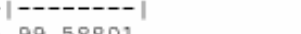 \\
\hline $2 \quad 26.320 \mathrm{BP}$ & 0.1179 & $\begin{array}{r}1.89099 \\
1.89099\end{array}$ & $2.00804 e-1$ & $\begin{array}{r}99.58801 \\
0.41199\end{array}$ \\
\hline Totals : & & 458.98535 & 37.32266 & \\
\hline
\end{tabular}

$\star \star \star$ End of Report $* \star *$ 


\section{Table entry 2}

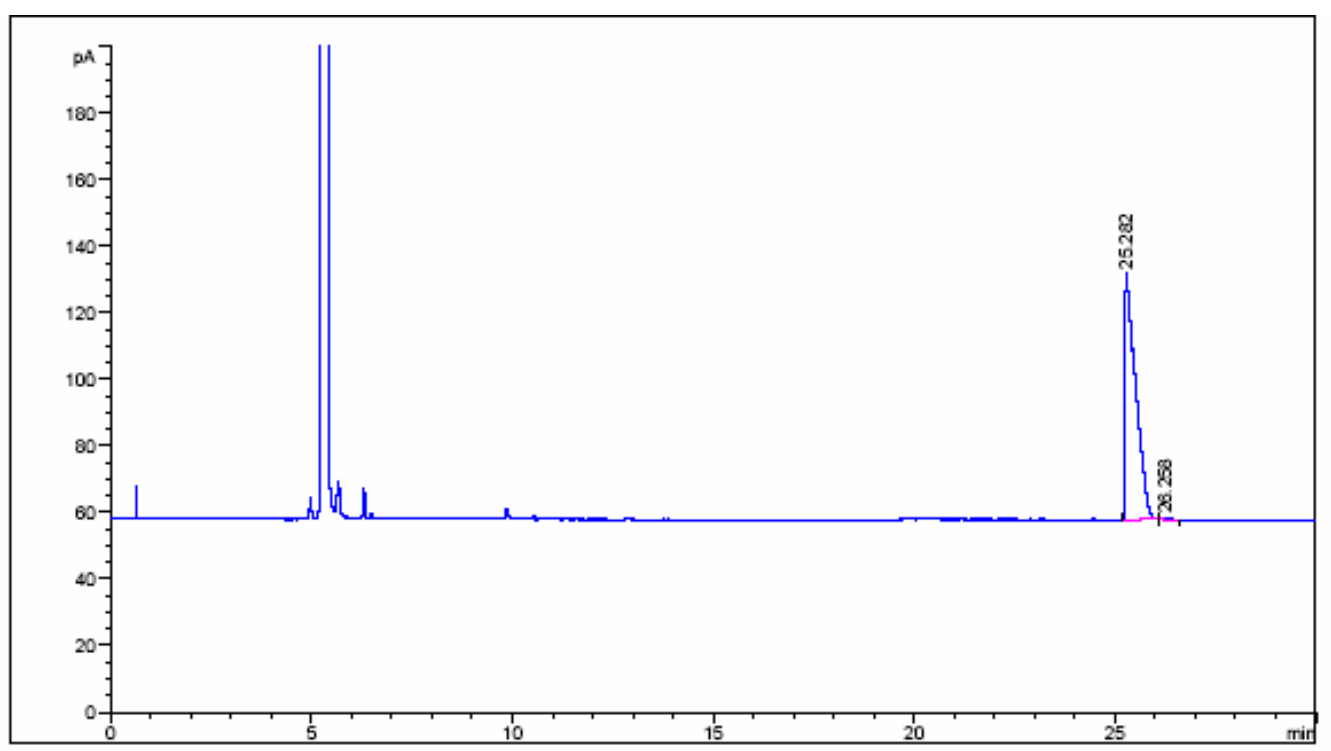

Area Percent Report

Sorted By : : Signal

Multiplier : $\quad 1.0000$

Dilution $\quad: \quad 1.0000$

Sample Amount 1.00000 [ng/ul] (not used in calc.)

Signal 1: FID1 $\boldsymbol{A}$,

Peak RetTime Type width Area Height Area

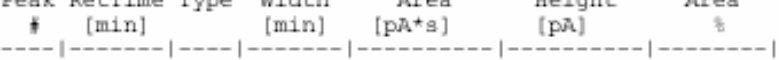

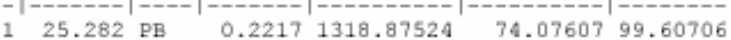

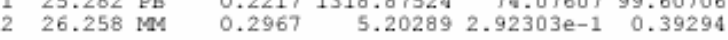

Totals : $\quad 1324.07813 \quad 34.36838$

Results obtained with enhanced integrator!

$\star \star \star$ End of Report *** 


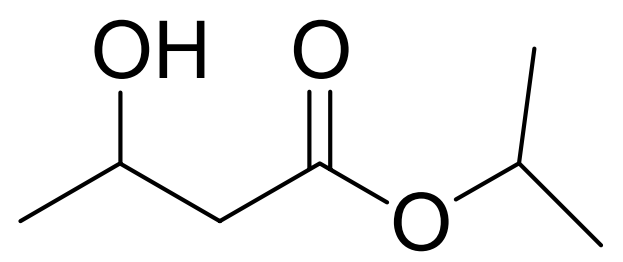

\section{$8 b$ racemate}

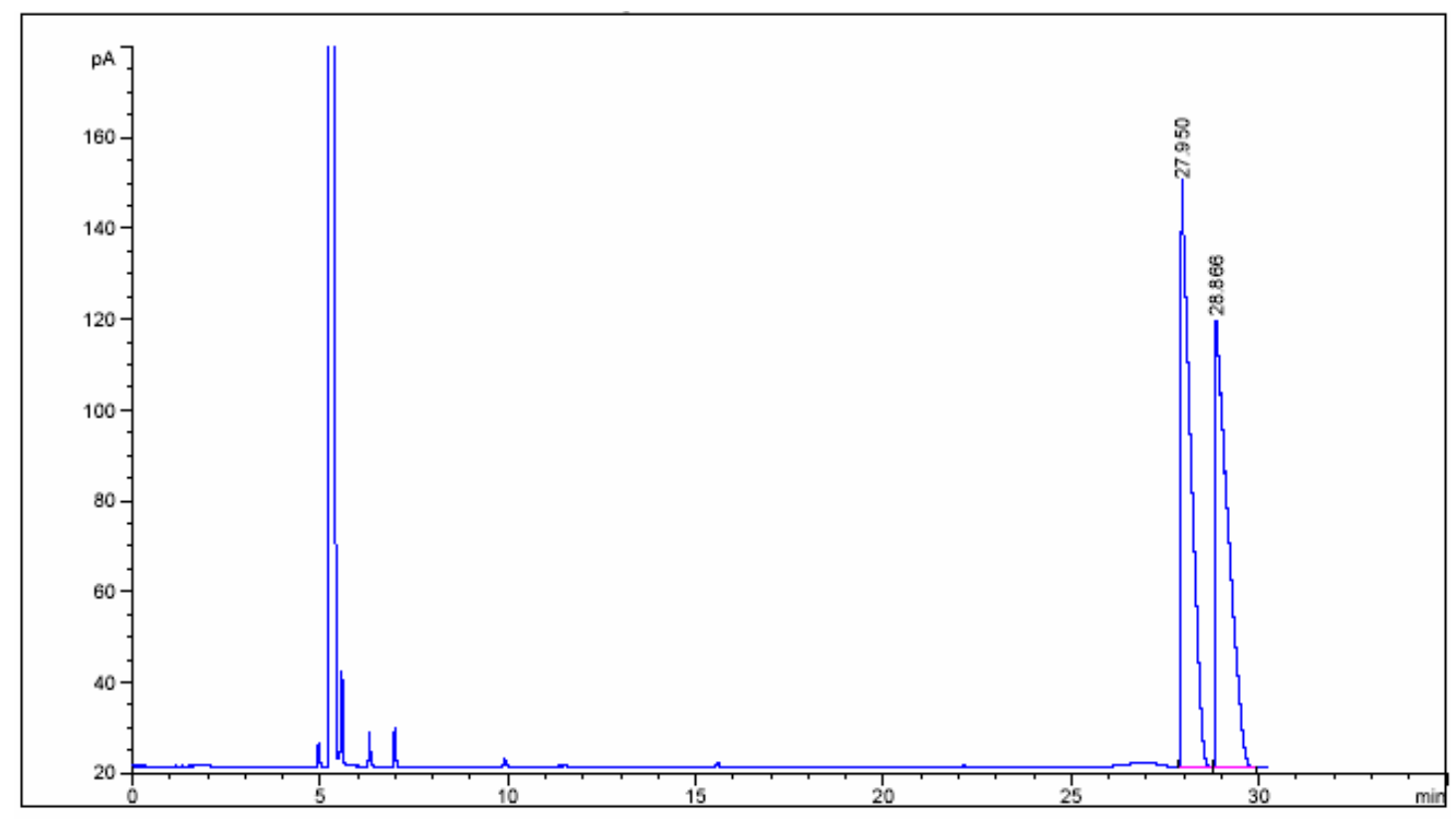

Area Percent Report

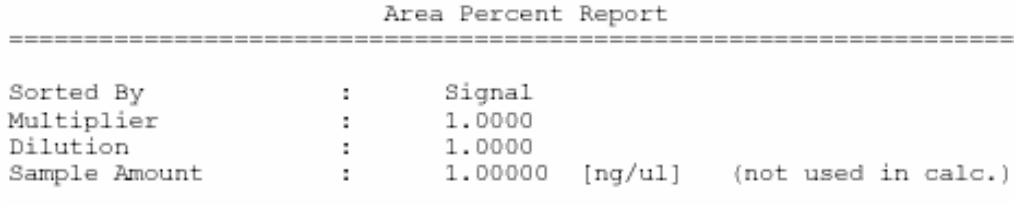

Signal 1: FID1 A,

\begin{tabular}{|c|c|c|c|c|c|c|}
\hline $\begin{array}{c}\text { Peak } \\
\#\end{array}$ & $\begin{array}{l}\text { RetTime } \\
\text { [min] }\end{array}$ & Type & $\begin{array}{l}\text { Width } \\
\text { [min] }\end{array}$ & $\begin{array}{r}\text { Area } \\
{\left[\mathrm{pA}^{*} \mathrm{~s}\right]}\end{array}$ & $\begin{array}{l}\text { Height } \\
\text { [pA] }\end{array}$ & $\begin{array}{c}\text { Area } \\
\qquad 8\end{array}$ \\
\hline 1 & & & 0.2274 & 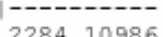 & 12851300 & 50.01782 \\
\hline $\begin{array}{l}1 \\
2\end{array}$ & 28.866 & VB & 0.2871 & $\begin{array}{r}2284.10986 \\
2282.48193\end{array}$ & $\begin{array}{r}128.51309 \\
98.24083\end{array}$ & 49.98218 \\
\hline Tota. & : & & & 4566.59180 & 226.75392 & \\
\hline
\end{tabular}

Results obtained with enhanced integrator!

$\star * *$ End of Report $* * *$ 


\section{Table entry 3}

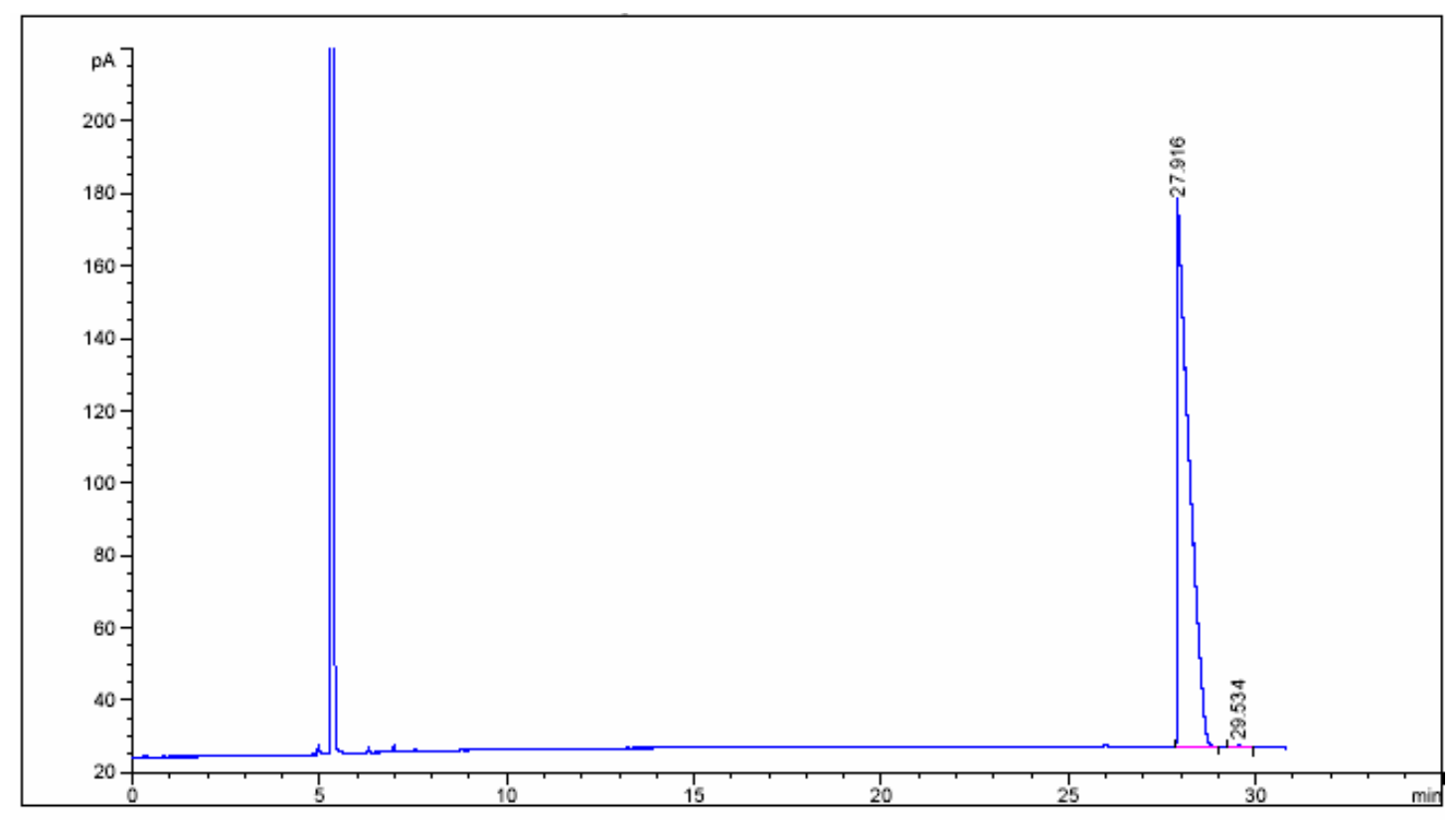

Area Percent Report

\begin{tabular}{|c|c|c|c|c|}
\hline Sorted By & : & Signal & & \\
\hline Multiplier & : & 1.0000 & & \\
\hline Dilution & : & 1.0000 & & \\
\hline Sample Amount & : & 1.00000 & {$[\mathrm{ng} / \mathrm{u} 1]$} & (not used \\
\hline \multicolumn{5}{|l|}{ Signal 1: FID1 A, } \\
\hline $\begin{array}{c}\text { Peak RetTime Type } \\
\# \quad \text { [min] }\end{array}$ & $\begin{array}{l}\text { Width } \\
\text { [min] }\end{array}$ & $\begin{array}{r}\text { Area } \\
{\left[p A^{\star} s\right]}\end{array}$ & $\begin{array}{l}\text { Height } \\
\text { [pA] }\end{array}$ & $\begin{array}{c}\text { Area } \\
\text { \& }\end{array}$ \\
\hline $127.916 \mathrm{~PB}$ & 0.2693 & 3353.64575 & 150.88533 & 99.77046 \\
\hline $29.534 \mathrm{PP}$ & 0.1695 & 7.71579 & $5.50421 e-1$ & 0.22954 \\
\hline Totals : & & 3361.36154 & 151.43575 & \\
\hline
\end{tabular}

Results obtained with enhanced integrator!

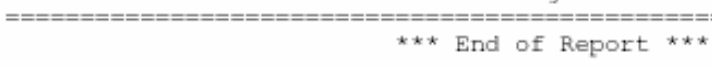




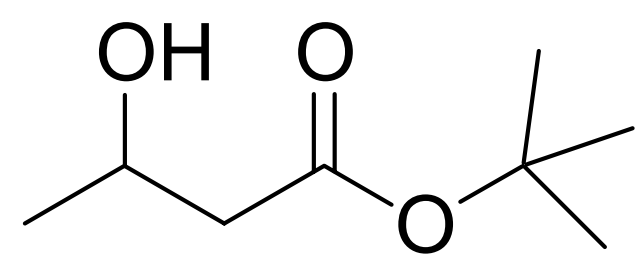

\section{$8 c$ racemate}

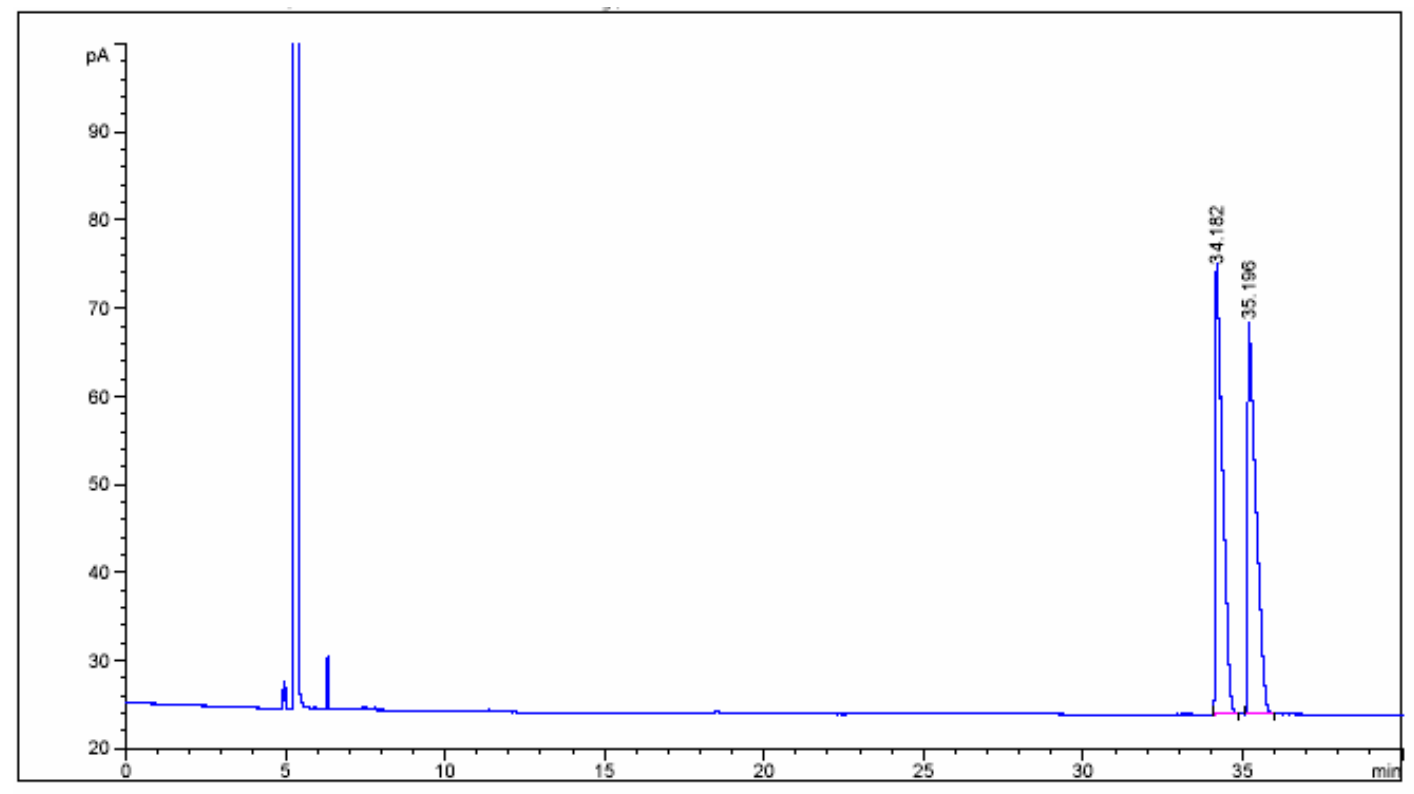

Area Percent Report

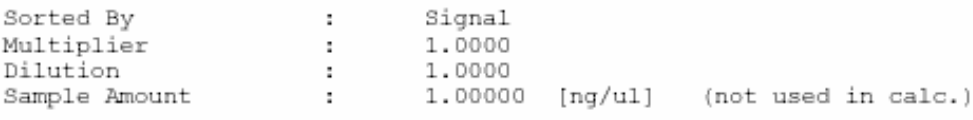

Signal 1: FID1 A,

\begin{tabular}{|c|c|c|c|c|c|c|}
\hline $\begin{array}{l}\text { Peak } \\
\#\end{array}$ & $\begin{array}{l}\text { RetTime } \\
\text { [min] }\end{array}$ & Type & $\begin{array}{l}\text { Width } \\
\text { [min] }\end{array}$ & $\begin{array}{r}\text { Area } \\
{\left[p^{*} s\right]}\end{array}$ & $\begin{array}{l}\text { Height } \\
\text { [pA] }\end{array}$ & $\begin{array}{c}\text { Area } \\
\qquad 8\end{array}$ \\
\hline 1 & 34.182 & $\mathrm{BB}$ & 0.2111 & 793.70892 & 50.58587 & 50.00264 \\
\hline 2 & 35.196 & $\mathrm{BB}$ & & 793.62524 & 44.14273 & 49.99736 \\
\hline ta & & & & 1587.33417 & 94.72860 & \\
\hline
\end{tabular}

Results obtained with enhanced integrator!

$* * *$ End of Report $* * *$ 


\section{Table1 entry4}

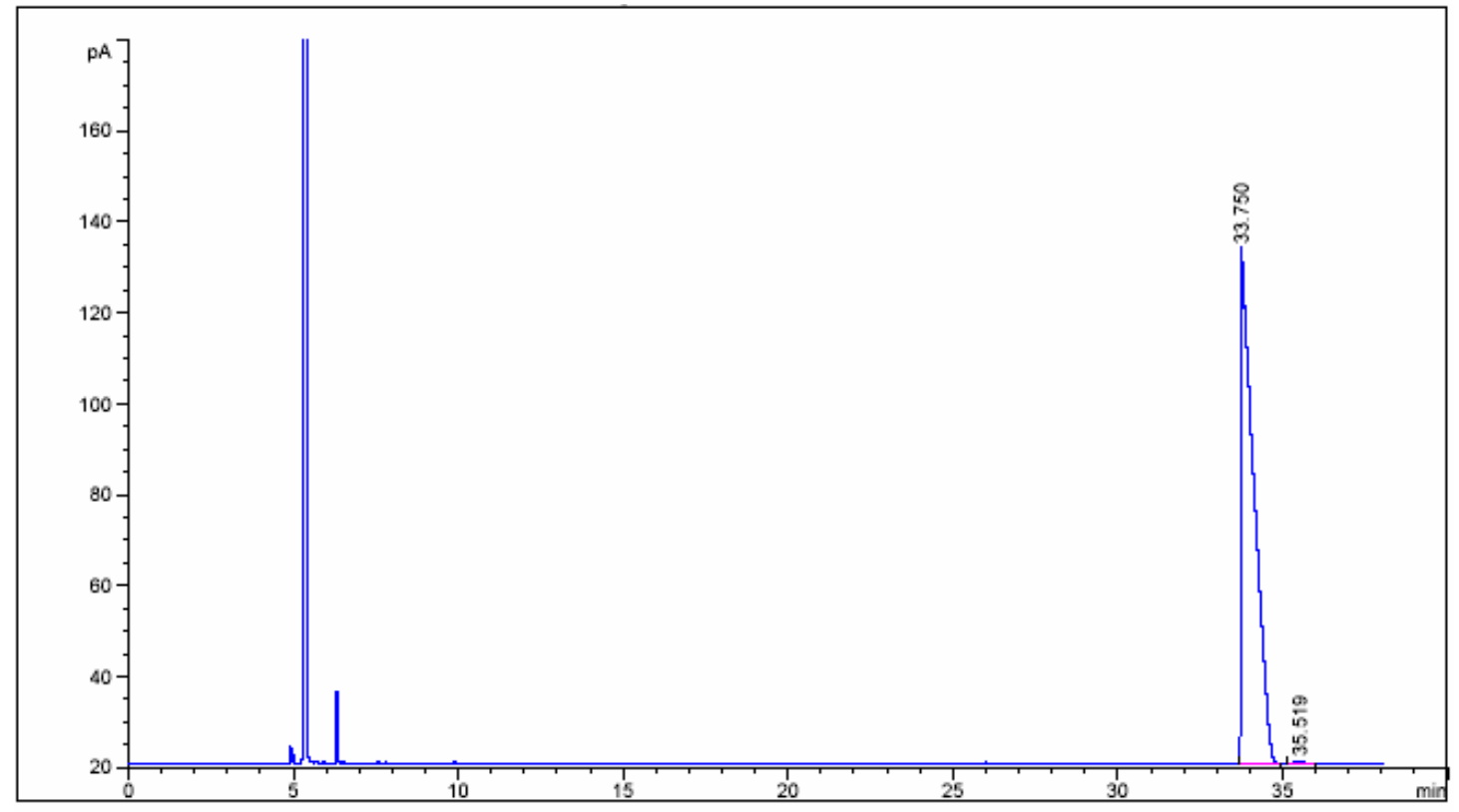

Area Percent Report

$\begin{array}{llll}\text { Sorted By } & : & \text { Signal } & \\ \text { Multiplier } & : & 1.0000 & \\ \text { Dilution } & : & 1.0000 & \\ \text { Sample Amount } & : & 1.00000 \text { [ng/ul] (not used in calc.) }\end{array}$

Signal 1: FID1 A,

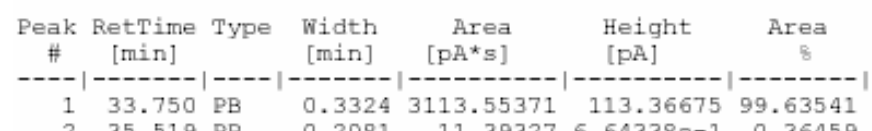

$\begin{array}{lllllll}2 & 35.519 \mathrm{PP} & 0.2081 & 11.39327 & 6.64338 \mathrm{e}-1 & 0.36459\end{array}$

Totals :

$3124.94698 \quad 114.03109$

Results obtained with enhanced integrator!

$\star * *$ End of Report *** 


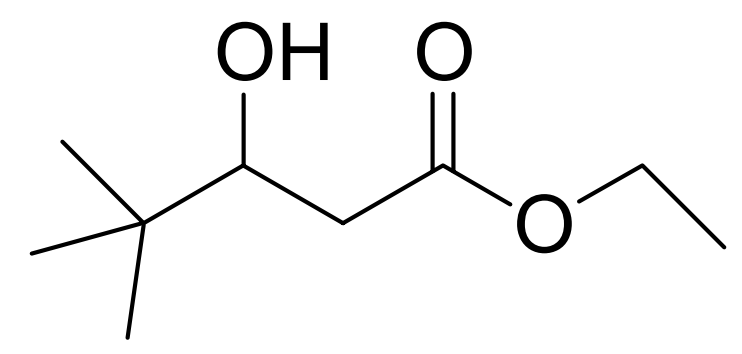

$8 d$ racemate

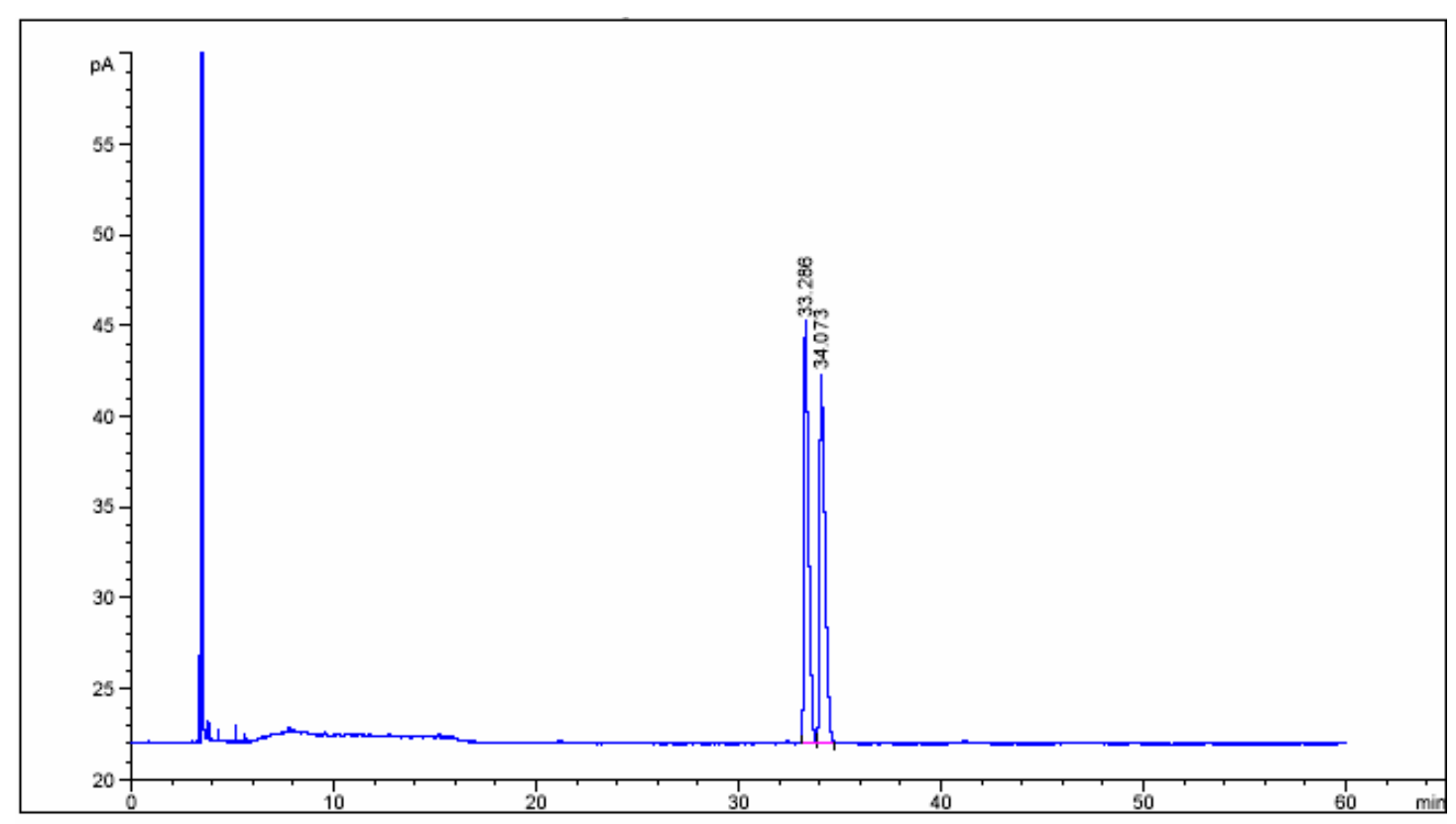

Area Percent Report

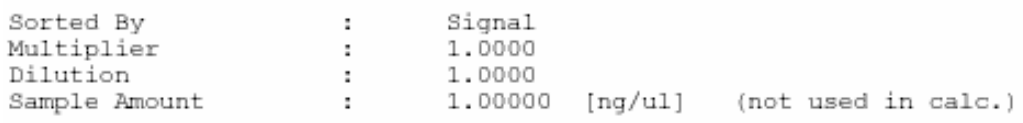

Signal 1: FID1 A,

\begin{tabular}{|c|c|c|c|c|c|c|}
\hline $\begin{array}{c}\text { Peak } \\
\#\end{array}$ & $\begin{array}{l}\text { RetTime } \\
\text { [min] }\end{array}$ & Type & $\begin{array}{l}\text { Width } \\
\text { [min] }\end{array}$ & $\begin{array}{r}\text { Area } \\
{\left[\mathrm{pA}^{*} \mathrm{~s}\right]}\end{array}$ & $\begin{array}{l}\text { Height } \\
\text { [pA] }\end{array}$ & $\begin{array}{c}\text { Area } \\
8\end{array}$ \\
\hline 1 & $1--1-1$ & & 0.2093 & 364.46973 & 23.19306 & $\overline{49.91851}$ \\
\hline $\begin{array}{l}1 \\
2\end{array}$ & 34.073 & $\begin{array}{l}\text { BV } \\
\text { VB }\end{array}$ & 0.2429 & 365.65967 & $\begin{array}{l}23.19300 \\
20.22982\end{array}$ & $\begin{array}{l}49.91851 \\
50.08149\end{array}$ \\
\hline ota & $1 \mathrm{~s}$ & & & 730.12939 & 43.42289 & \\
\hline
\end{tabular}

Results obtained with enhanced integrator!

$\star \star \star$ End of Report $\star \star *$ 


\section{Table1 entry5}

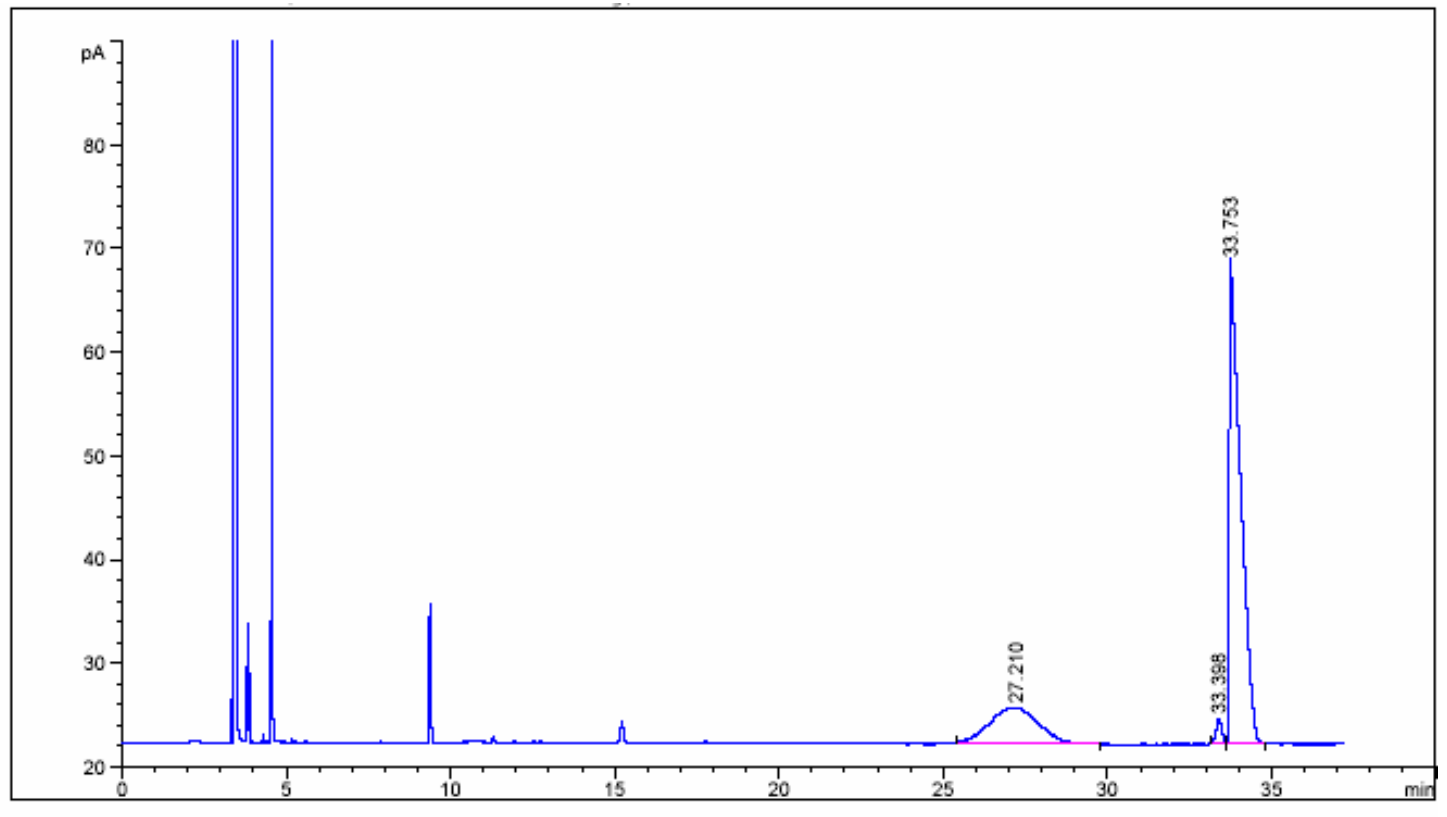

Area Percent Report

Sorted By

Multiplier

Dilution

Sample Amount

\section{Signa1}

1.0000

1.0000

1.00000 [ng/ul] (not used in calc.)

Signal 1: FID1 A,

\begin{tabular}{|c|c|c|c|c|c|c|}
\hline $\begin{array}{c}\text { Peak } \\
\#\end{array}$ & $\begin{array}{c}\text { RetTime } \\
\text { [min] }\end{array}$ & Type & $\begin{array}{l}\text { Width } \\
\text { [min] }\end{array}$ & $\begin{array}{r}\text { Area } \\
{\left[\mathrm{pA}^{*} \mathrm{~s}\right]}\end{array}$ & $\begin{array}{l}\text { Height } \\
{[p A]}\end{array}$ & $\begin{array}{c}\text { Area } \\
\text { g }\end{array}$ \\
\hline 1 & 27.210 & BB & 1.2111 & 356.75183 & 3.44791 & 23.73979 \\
\hline 2 & 33.398 & BV & 0.1839 & 29.16174 & 2.39744 & 1.94055 \\
\hline 3 & 33.753 & VB & 0.3084 & 1116.84546 & 46.47160 & 74.31966 \\
\hline
\end{tabular}

Totals :

$1502.75903 \quad 52.31695$

Results obtained with enhanced integrator!

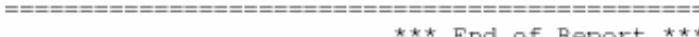




\section{Table1 entry6}

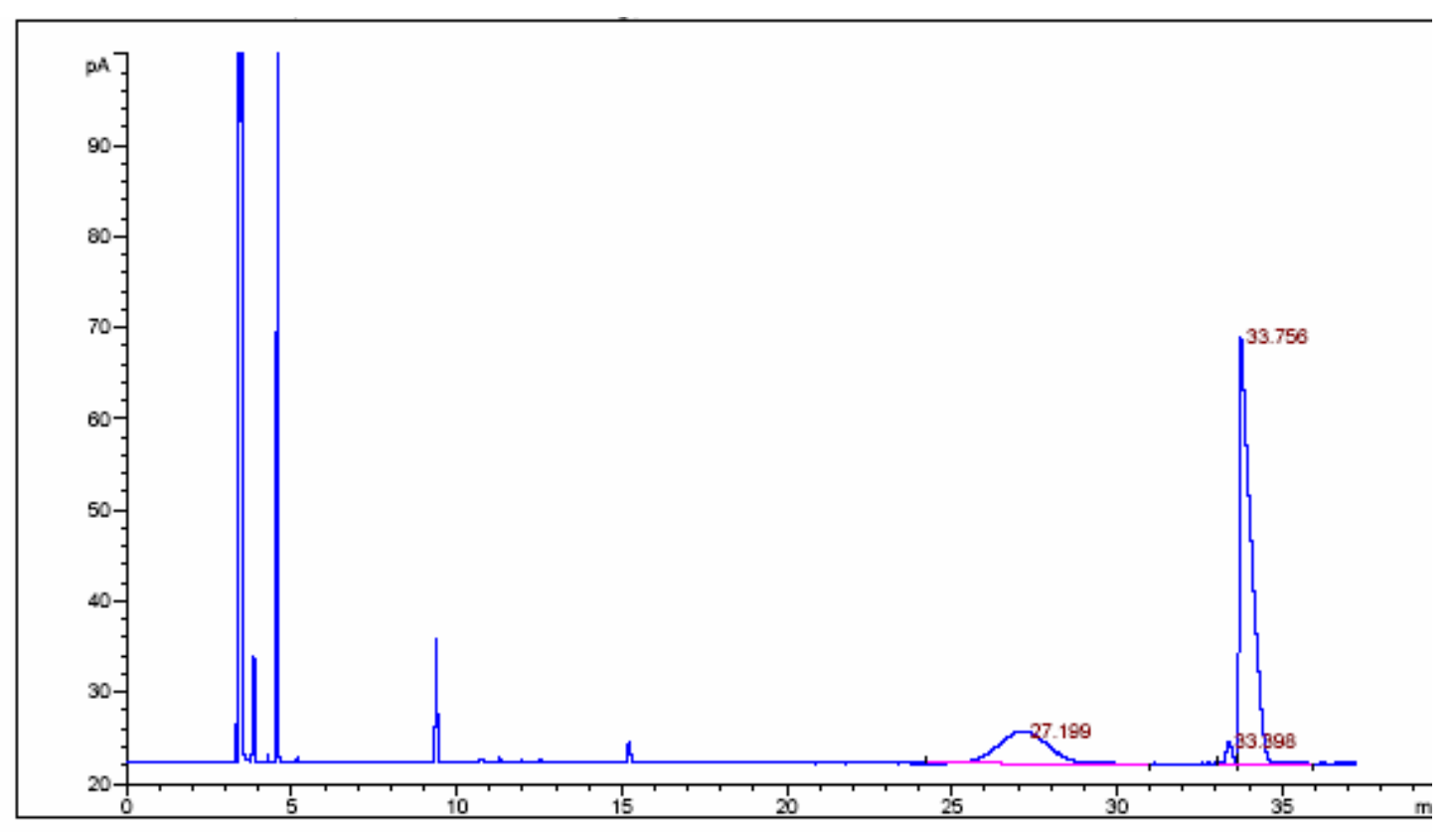

Rrea Percent Report

Sorted By : Signal

Calib. Data Modified : 02-12-10 10:54:30下午

Multiplie

Dilution

1.0000

Sample Amount

1.00000

[ng/ul) (not used in calc.)

Signal 1: FID1 A,

\begin{tabular}{|c|c|c|c|c|c|c|}
\hline $\begin{array}{c}\text { Peak } \\
\hbar\end{array}$ & $\begin{array}{l}\text { RetTime } \\
\text { [min] }\end{array}$ & Type & $\begin{array}{l}\text { Width } \\
\text { [min] }\end{array}$ & $\begin{array}{r}\text { Area } \\
{\left[p A^{\star} s\right]}\end{array}$ & $\begin{array}{c}\text { Area } \\
\text { g. }\end{array}$ & Name \\
\hline 1 & 2.021 & & 0.0000 & 0.00000 & 0.00000 & \\
\hline 2 & 2.131 & & 0.0000 & 0.00000 & 0.00000 & \\
\hline 3 & 2.271 & & 0.0000 & 0.00000 & 0.00000 & \\
\hline 4 & 2.353 & & 0.0000 & 0.00000 & 0.00000 & \\
\hline 5 & 2.513 & & 0.0000 & 0.00000 & 0.00000 & \\
\hline 6 & 2.626 & & 0.0000 & 0.00000 & 0.00000 & \\
\hline 3 & 2.698 & & 0.0000 & 0.00000 & 0.00000 & \\
\hline 8 & 3.026 & & 0.0000 & 0.00000 & 0.00000 & \\
\hline 9 & 27.199 & $M$ & 1.7886 & 378.69730 & 24.69345 & ? \\
\hline 10 & 33.398 & $M$ & 0.2063 & 30.10651 & 1.96313 & ? \\
\hline 11 & 33.756 & $M$ & 0.3992 & 1124.79028 & 33.34342 & ? \\
\hline
\end{tabular}




\section{Table1 entry7}

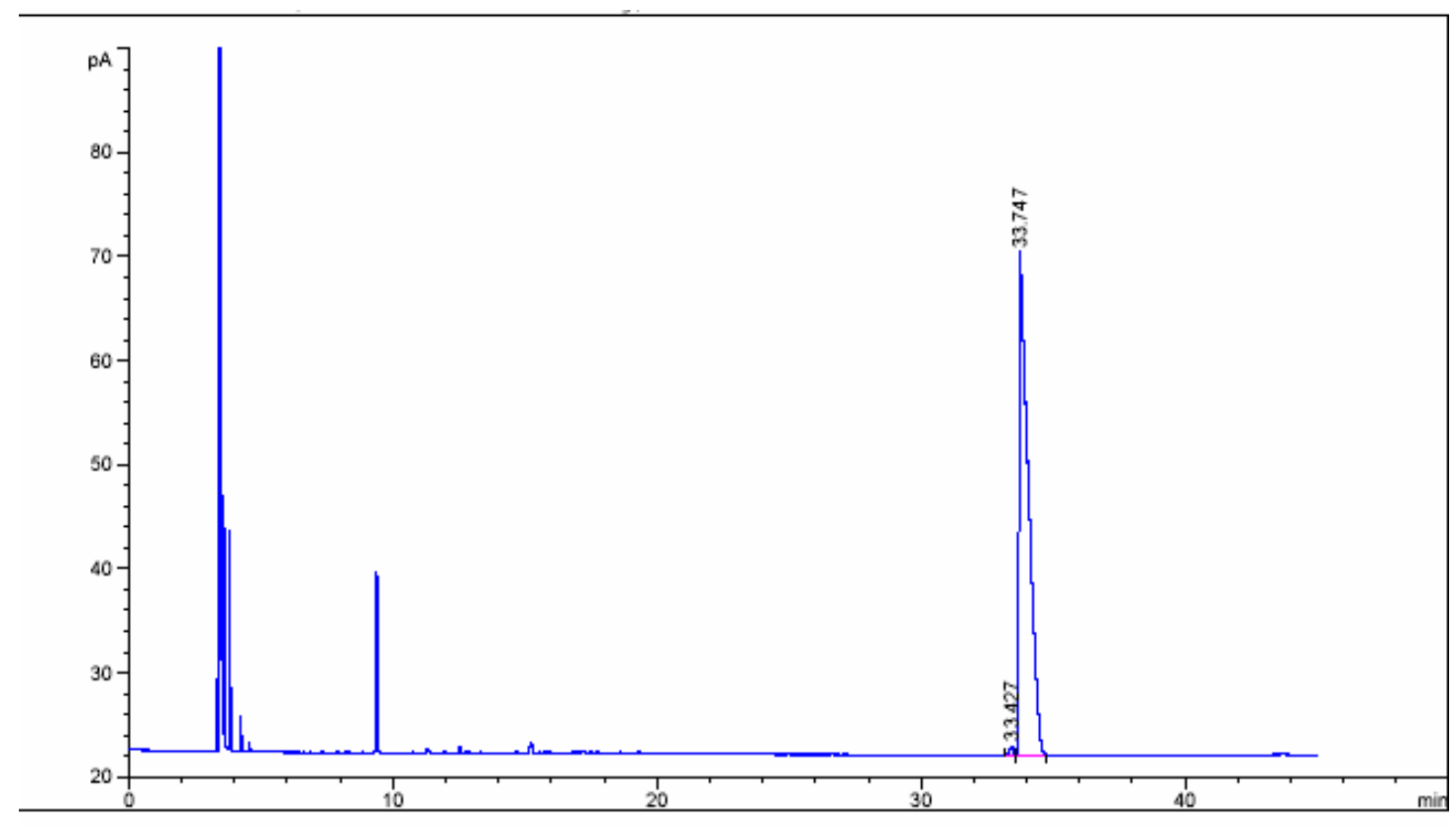

Area Percent Report

Sorted By

Multiplier

Dilution

Sample Amount

Signa1

1.0000

1.0000

1.00000 [ng/ul] (not used in calc.)

Signal 1: FID1 A,

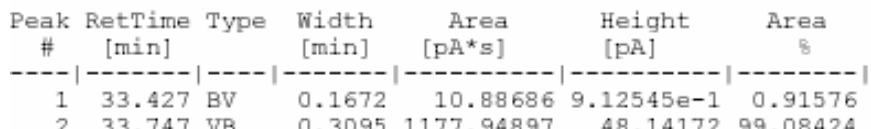

Totals :

$1188.83583 \quad 49.05426$

Results obtained with enhanced integrator!

$\star \star *$ End of Report $* * *$ 


\section{Table1 entry8}

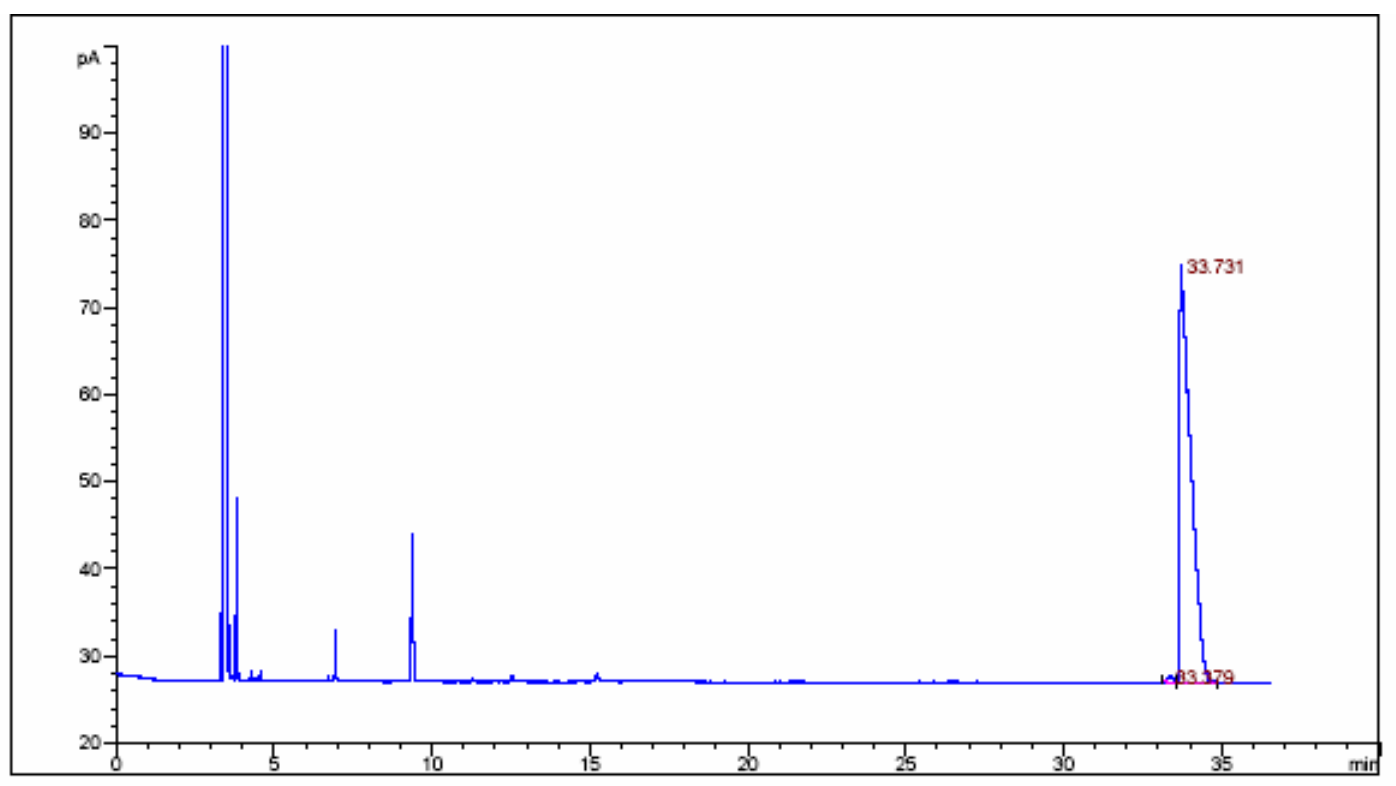

Area Percent Report

$\begin{array}{lcll}\text { Sorted By } & : & \text { Signal } & \\ \text { Calib. Data Modified } & : & 02-12-10 \text { 10:54:30 下午 } \\ \text { Multiplier } & \vdots & 1.0000 & \\ \text { Dilution } & \vdots & 1.0000 & \\ \text { Sample Amount } & : & 1.00000 \text { (ng/ul) (not used in calc.) }\end{array}$

Signal 1: FID1 $\boldsymbol{A}$,

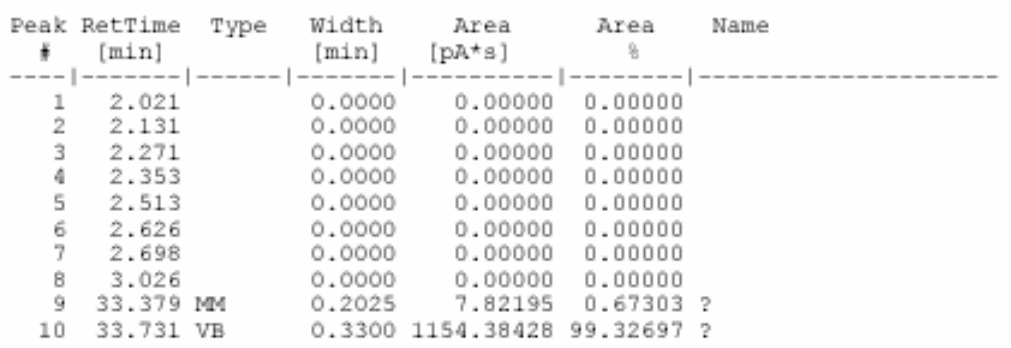




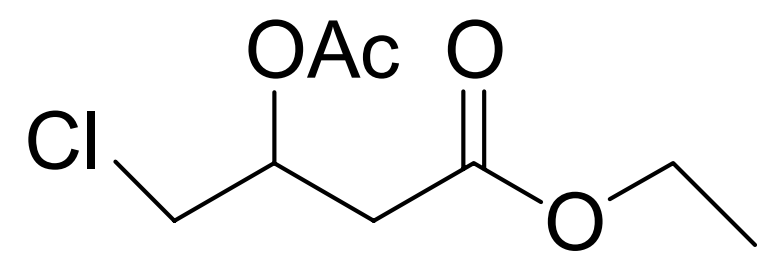

8 e racemate

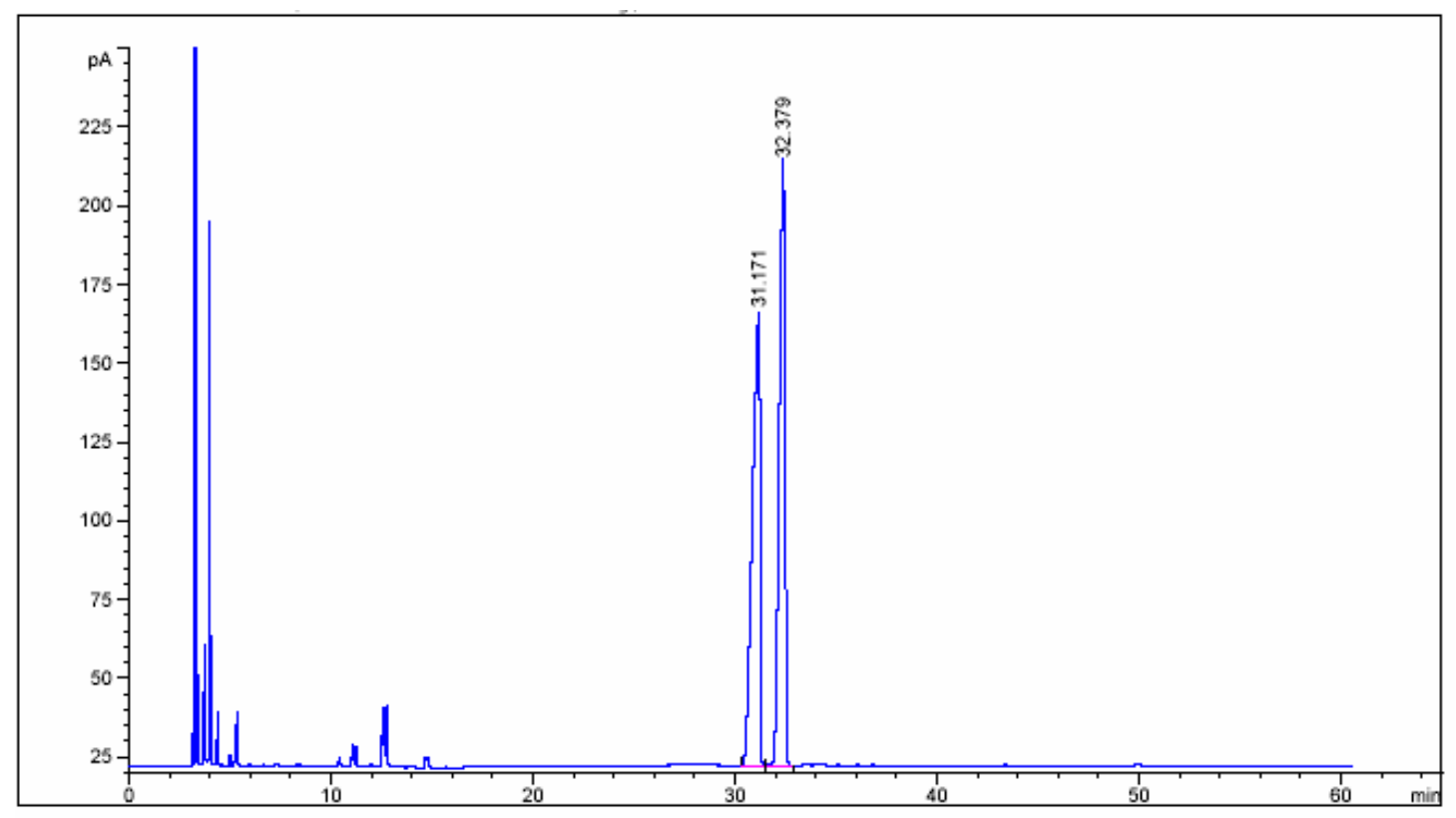

Area Percent Report

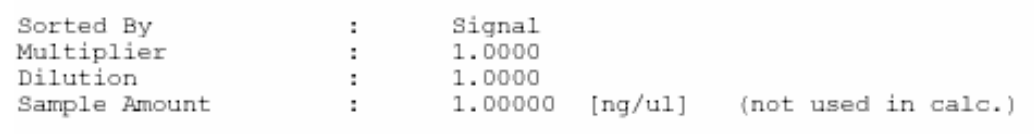

Signal 1: FID1 A,

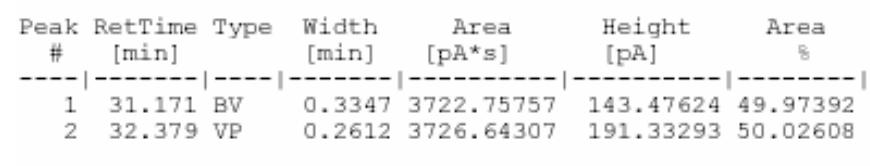

Totals :

$7449.40063 \quad 334.80917$

Results obtained with enhanced integrator!

$\star \star \star$ End of Report $* \star *$ 


\section{Table1 entry9}

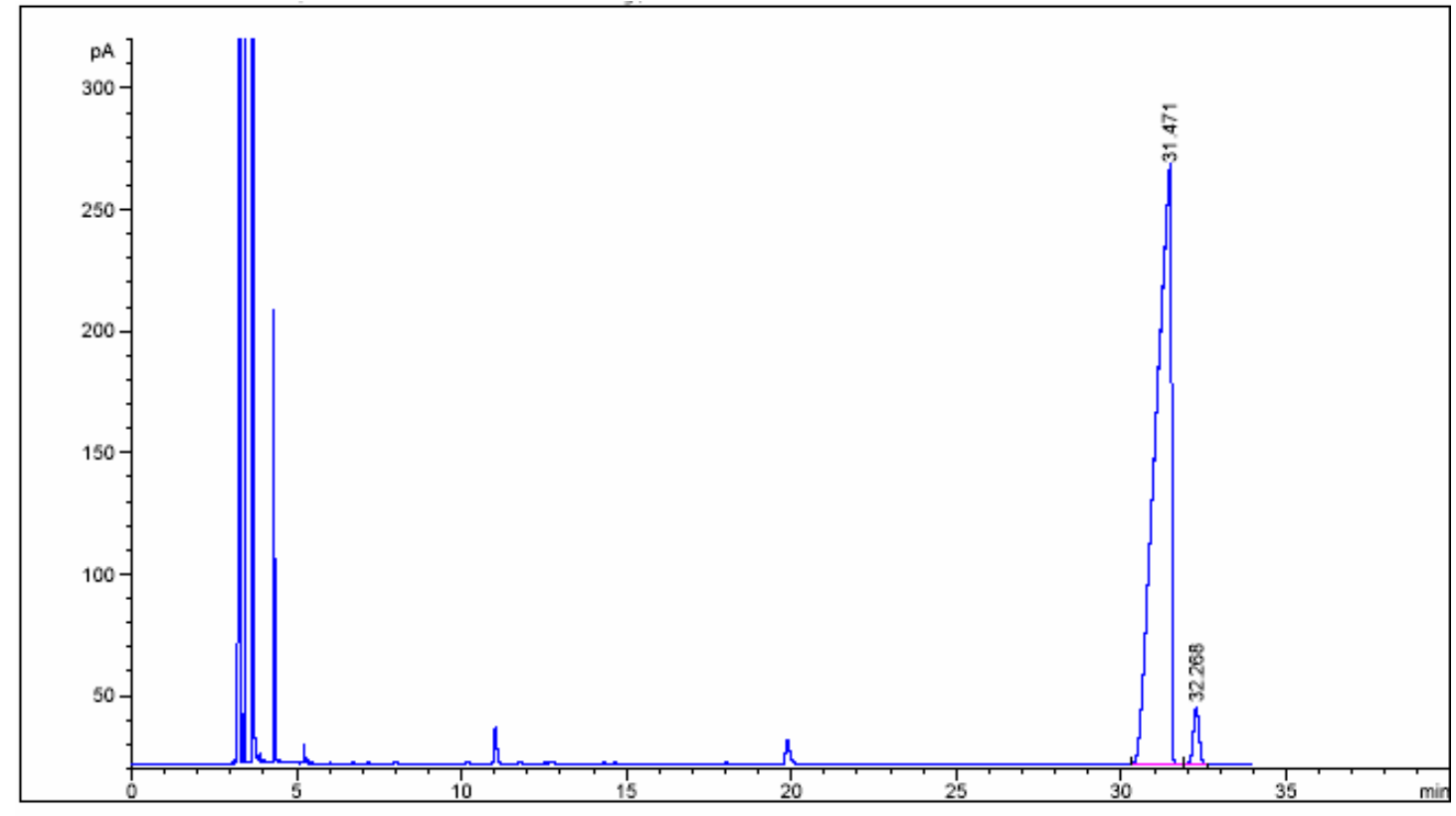

Area Percent Report

$\begin{array}{llll}\text { Sorted By } & : & \text { Signal } & \\ \text { Multiplier } & : & 1.0000 \\ \text { Dilution } & : & 1.0000 & \\ \text { Sample Amount } & : & 1.00000 \text { [ng/ul] (not used in calc.) }\end{array}$

Signal 1: FID1 A,

\begin{tabular}{|c|c|c|c|c|c|c|}
\hline $\begin{array}{c}\text { Peak } \\
\#\end{array}$ & $\begin{array}{c}\text { RetTime } \\
\text { [min] }\end{array}$ & Type & $\begin{array}{l}\text { Width } \\
\text { [min] }\end{array}$ & $\begin{array}{r}\text { Area } \\
{\left[p^{*} s\right]}\end{array}$ & $\begin{array}{l}\text { Height } \\
\text { [pA] }\end{array}$ & $\begin{array}{c}\text { Area } \\
\text { 8 }\end{array}$ \\
\hline 1 & 31.471 & BV & 0.4121 & 8087.93115 & 245.64191 & 96.63807 \\
\hline 2 & 32.268 & VB & 0.1936 & 281.36978 & 22.85906 & 3.36193 \\
\hline ta. & : & & & 8369.30093 & 268.50097 & \\
\hline
\end{tabular}

Results obtained with enhanced integrator! 


\section{Table1 entry 10}

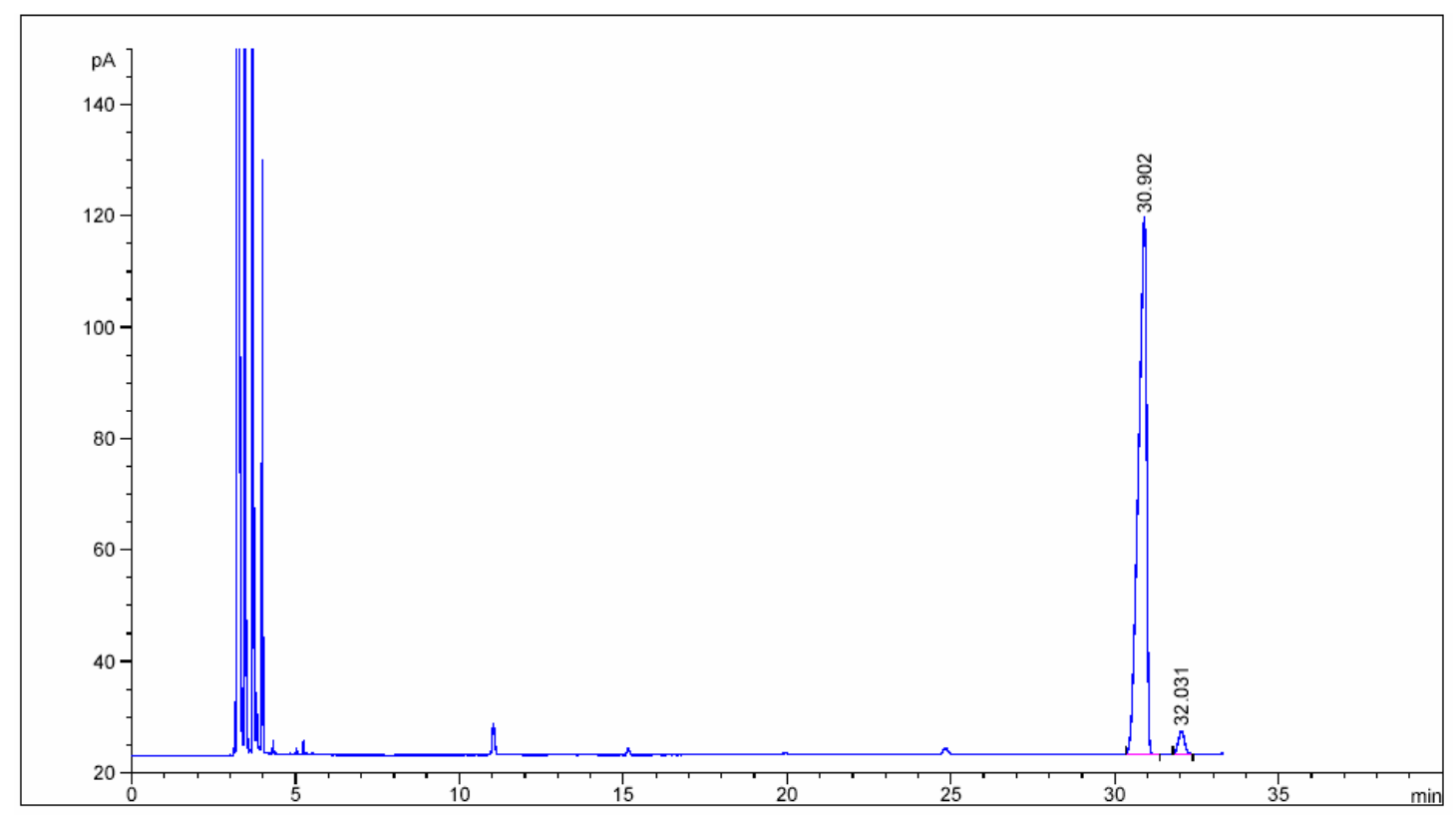

Area Percent Report

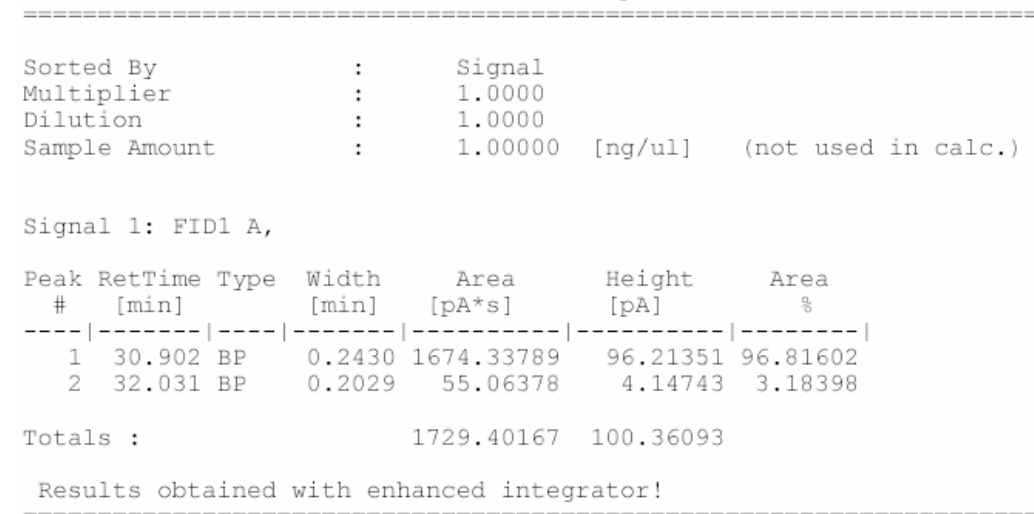

*** End of Report *** 


\section{Table1 entry 11}

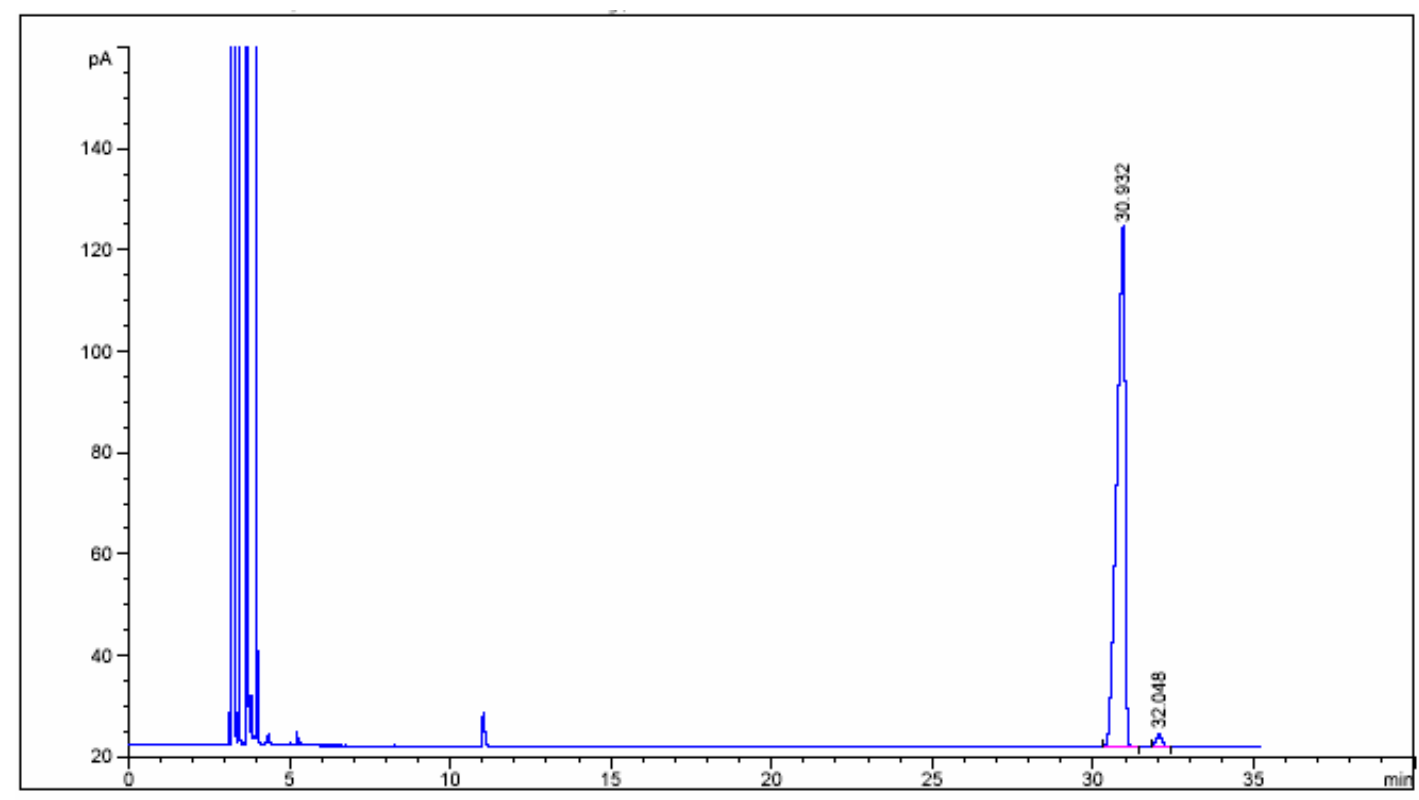

Area Percent Report

$\begin{array}{llll}\text { Sorted By } & : & \text { Signal } & \\ \text { Multiplier } & : & 1.0000 & \\ \text { Dilution } & : & 1.0000 & \\ \text { Sample Amount } & : & 1.00000 \quad[\mathrm{ng} / \mathrm{ul}] \quad \text { (not used in calc.) }\end{array}$

Signal 1: FID1 A,

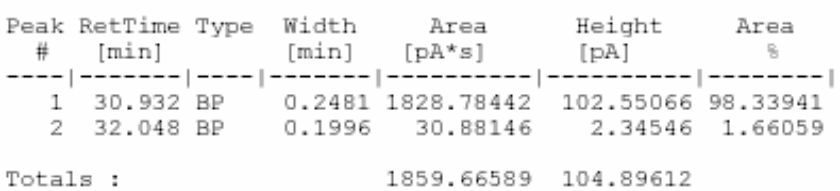

hanced integrator!

$* * \star$ End of Report $* * *$
. 


\section{Table 1 entry 12}

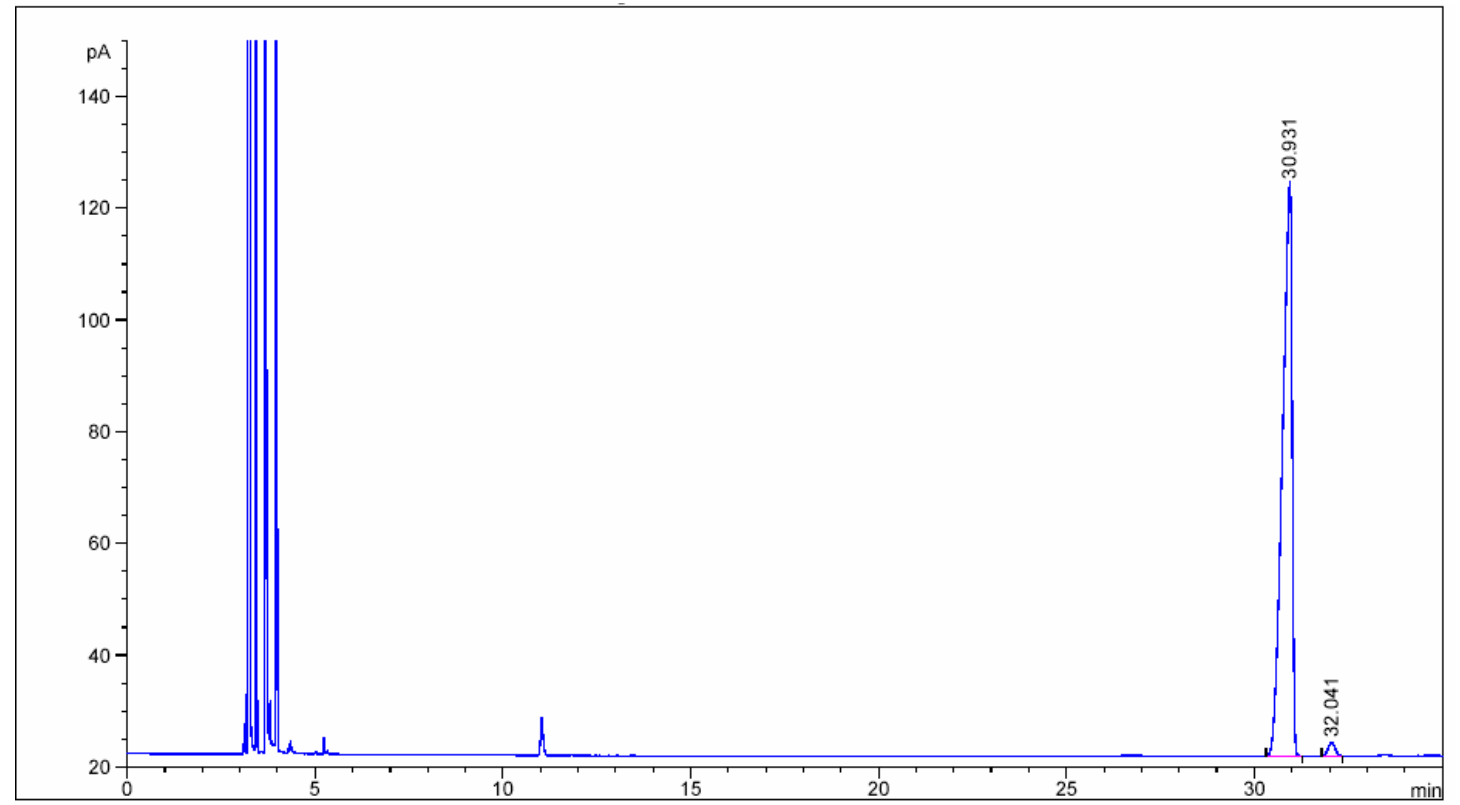

Area Percent Report

Sorted By

Multiplier

Dilution

Sample Amount

Signal

1.0000

1.0000

1.00000 [ng/ul] (not used in calc.

Signal 1: FID1 A,

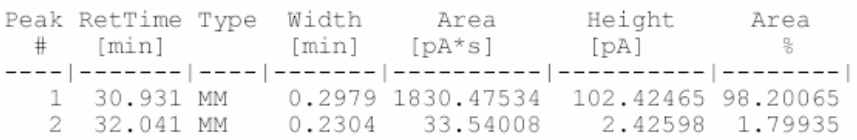

Totals :

$1864.01543 \quad 104.85063$

Results obtained with enhanced integrator! 


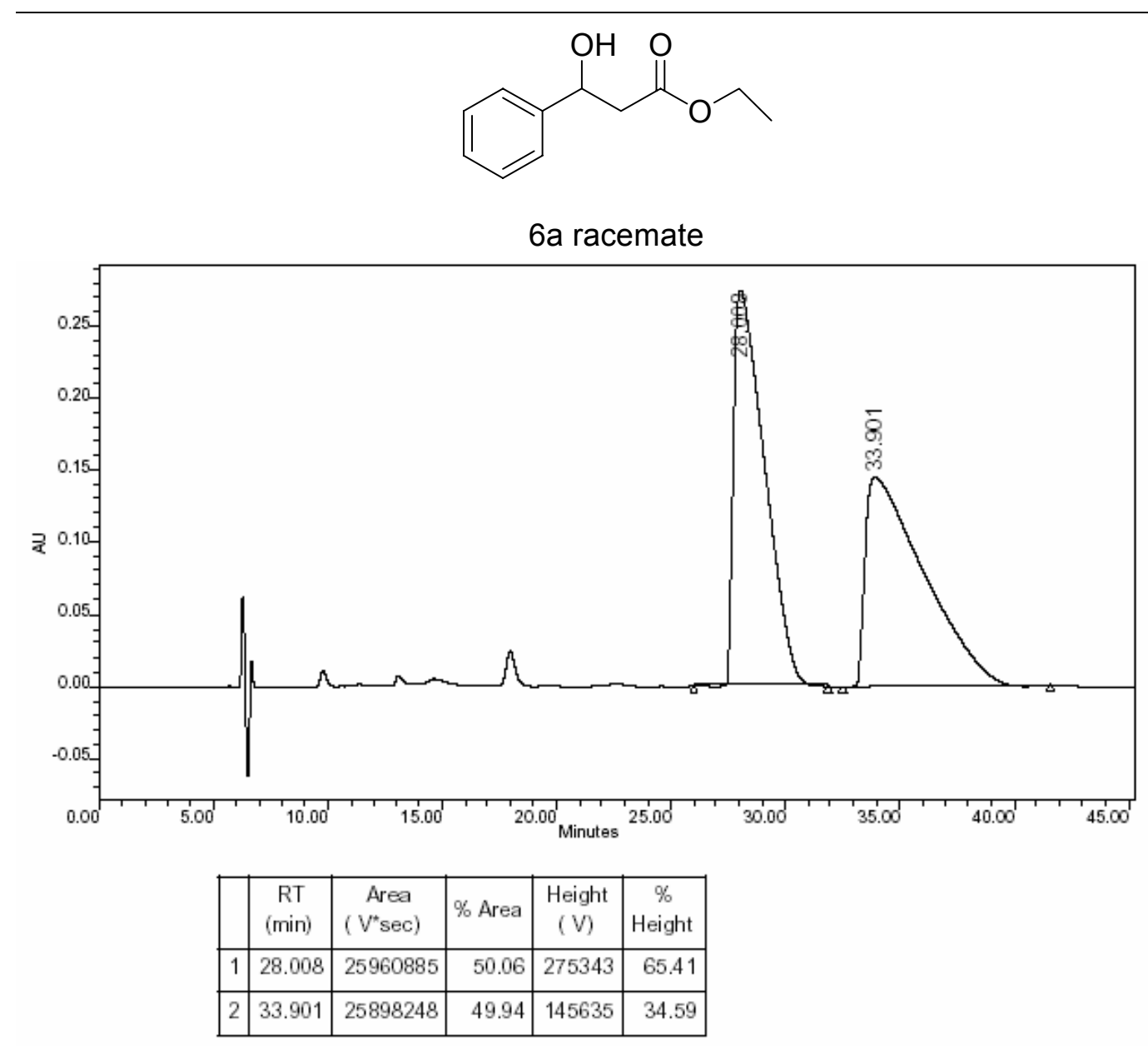

Table2 entry1

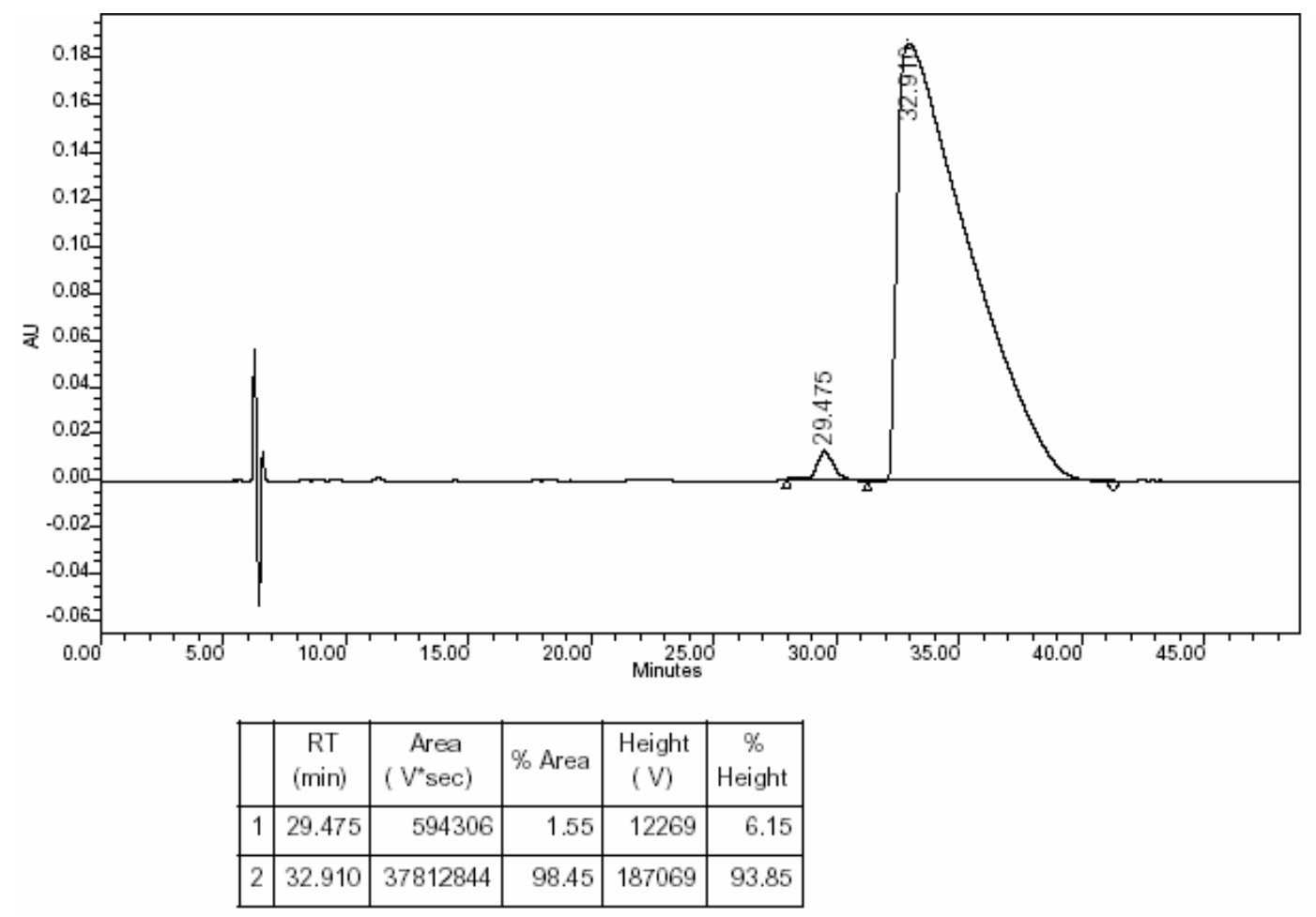




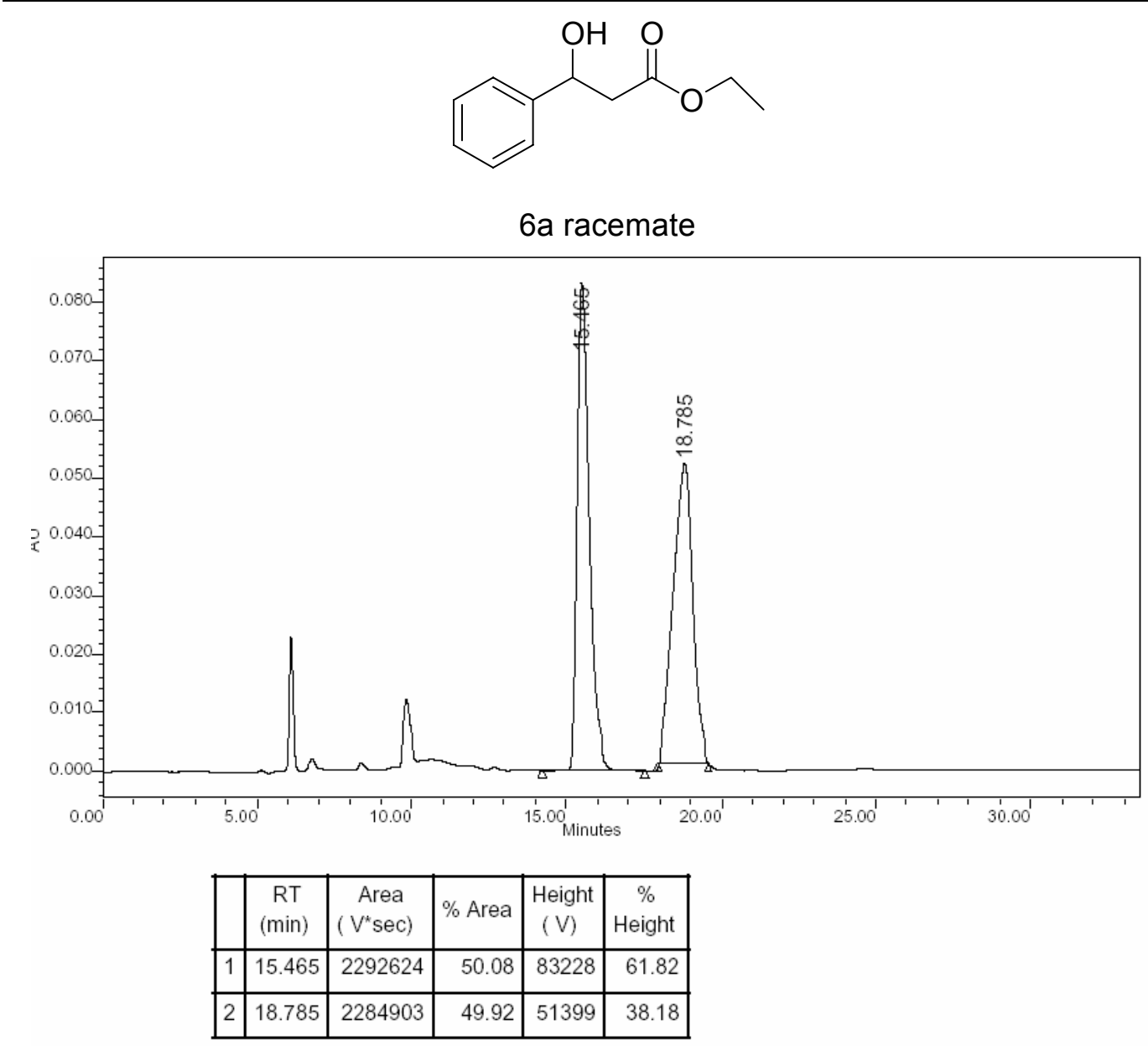

\section{Table 2 entry 2}

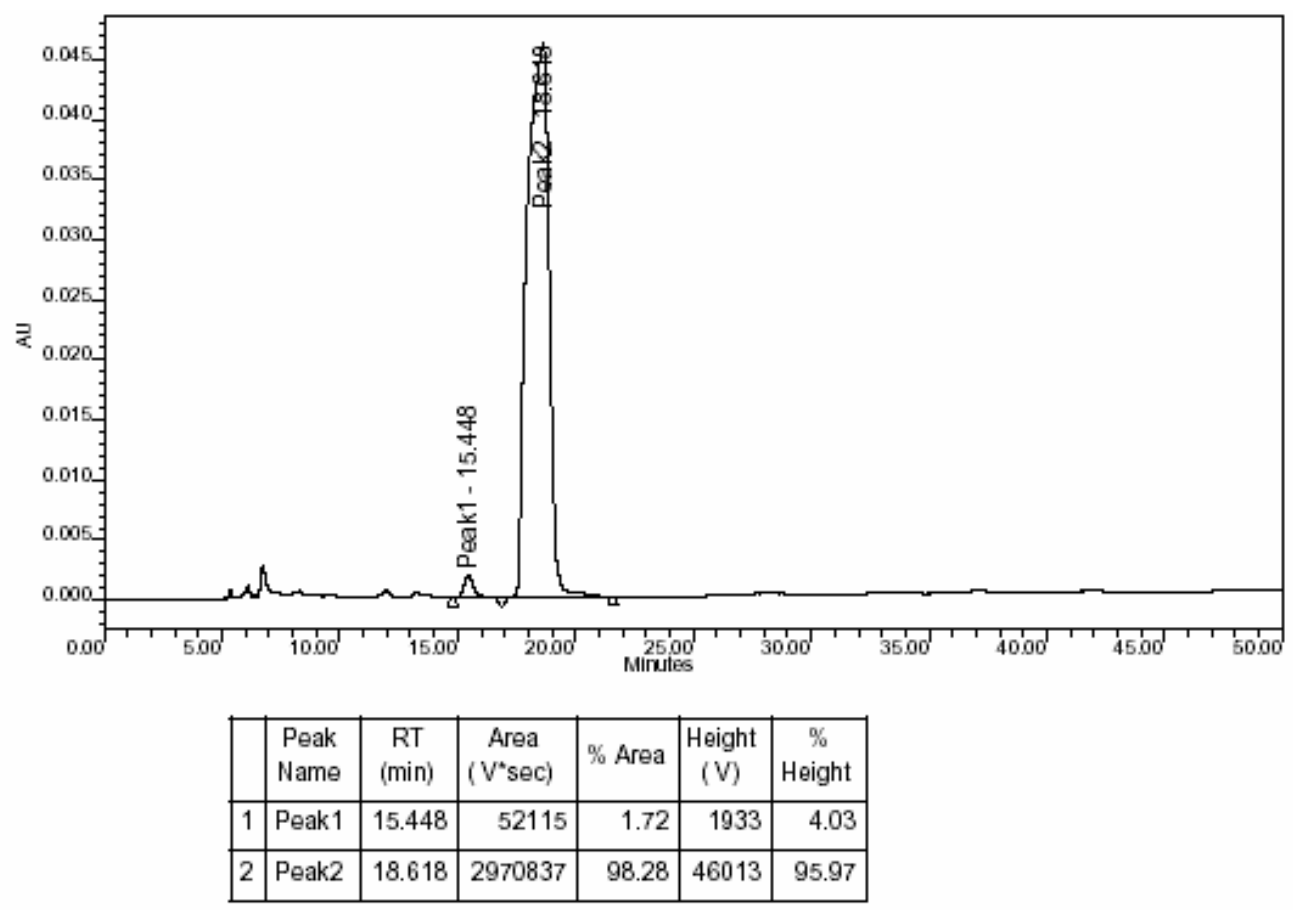


<smiles>CCOC(=O)CC(O)c1ccc(OC)cc1</smiles>

$6 \mathrm{~b}$ racemate

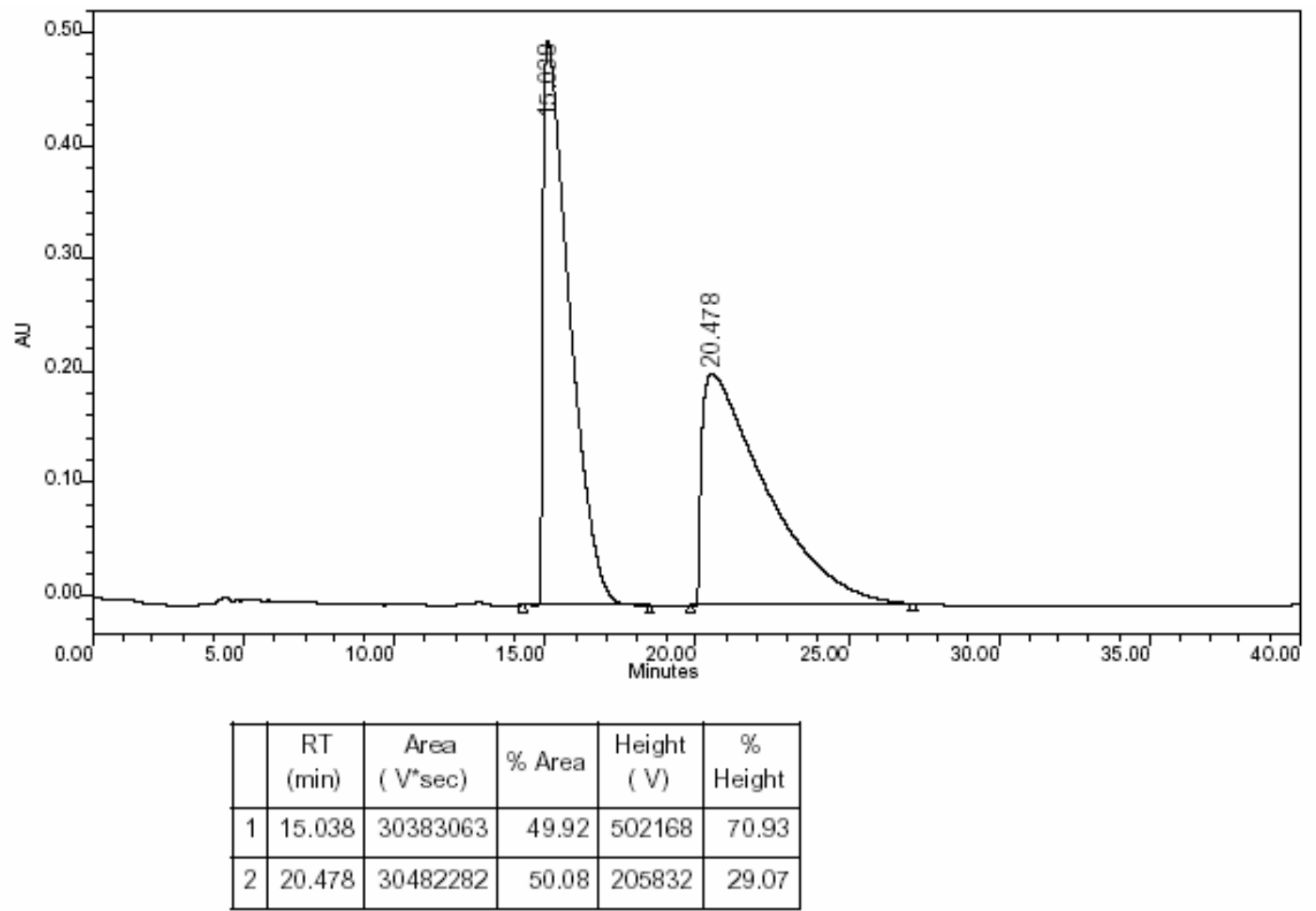




\section{Table 2 entry 3}

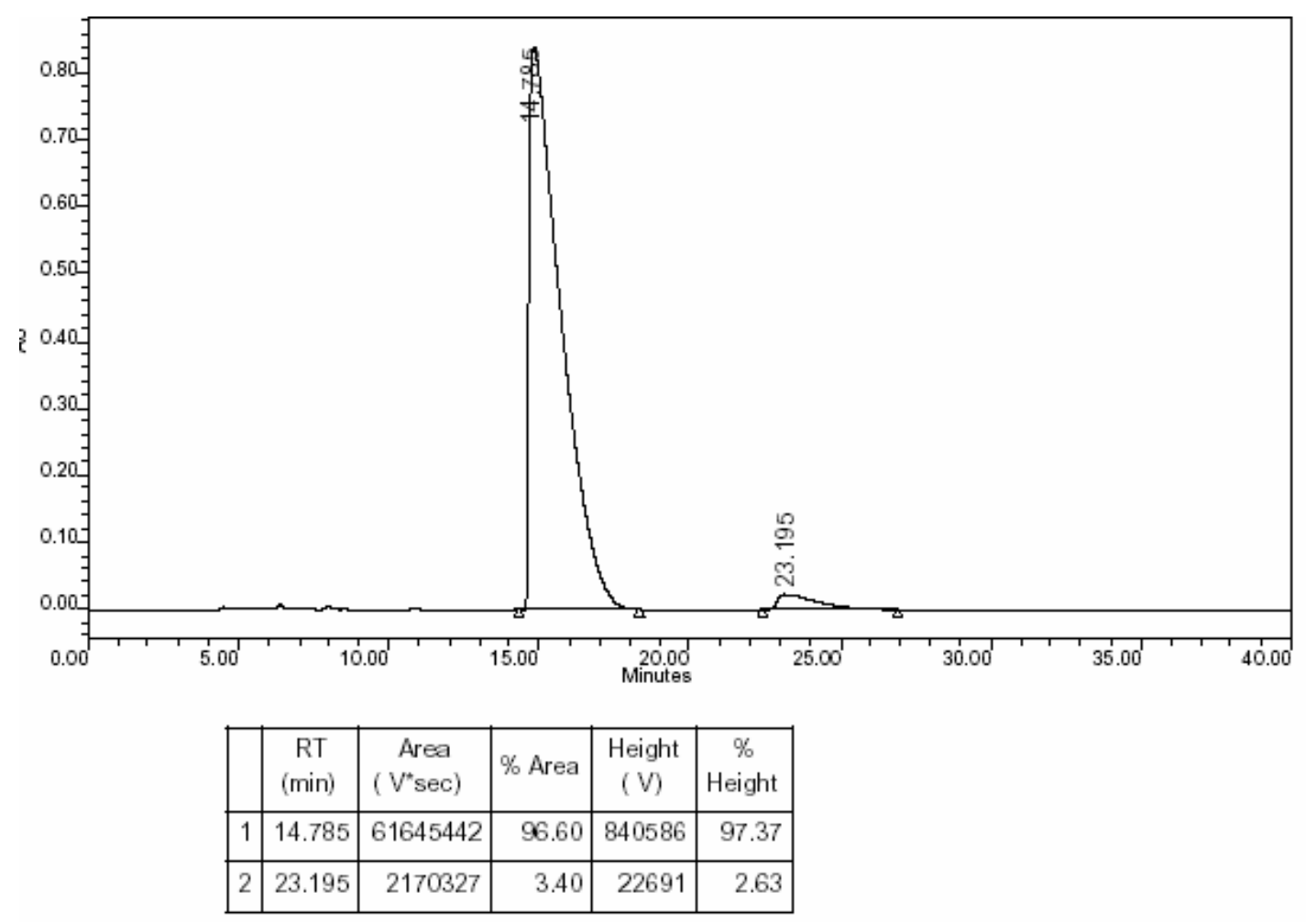

\section{Table 2 entry 4}

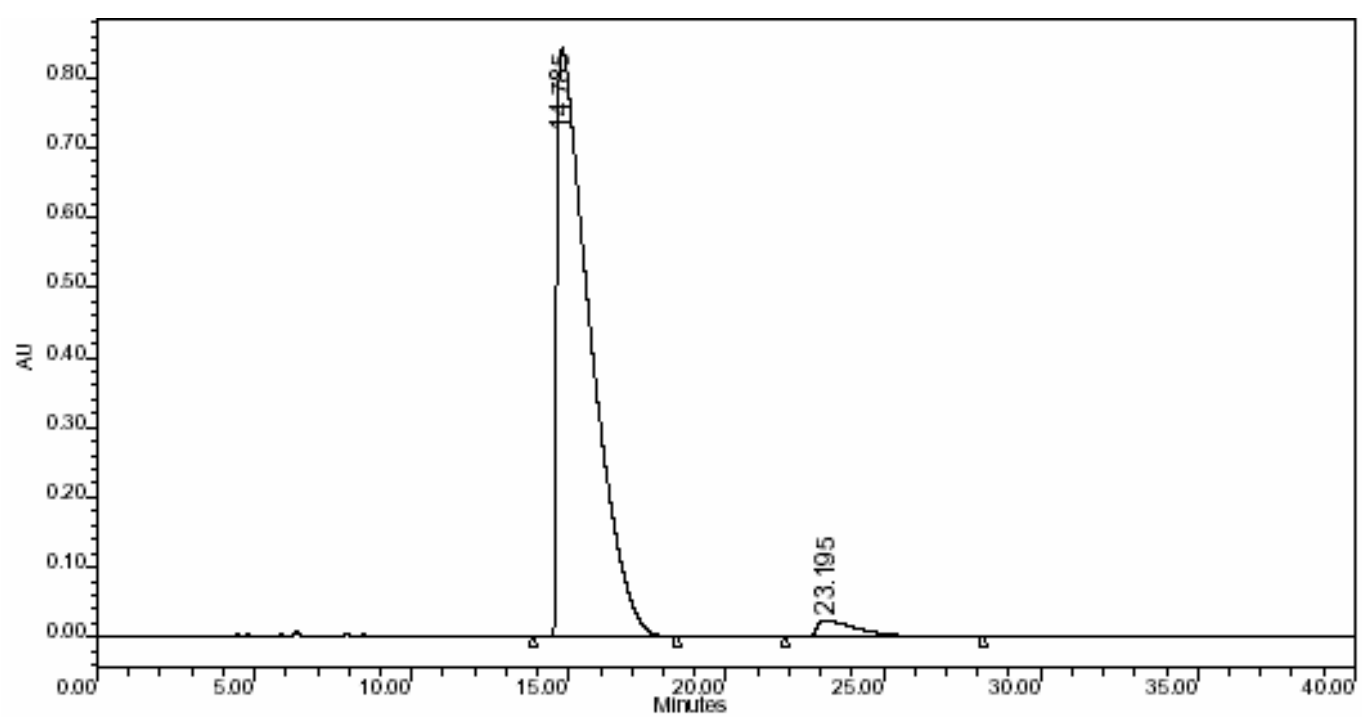

\begin{tabular}{|c|c|c|r|r|r|}
\hline & $\begin{array}{c}\text { RT } \\
\text { (min) }\end{array}$ & $\begin{array}{c}\text { Area } \\
\left(V^{*} \text { sec }\right)\end{array}$ & \% Area & $\begin{array}{c}\text { Height } \\
\text { (V) }\end{array}$ & $\begin{array}{c}\% \\
\text { Height }\end{array}$ \\
\hline 1 & 14.785 & 61740066 & 96.51 & 841195 & 97.35 \\
\hline 2 & 23.195 & 2234144 & 3.49 & 22937 & 2.65 \\
\hline
\end{tabular}


<smiles>CCOC(=O)CC(O)c1ccc(C)cc1</smiles>

$6 c$ racemate

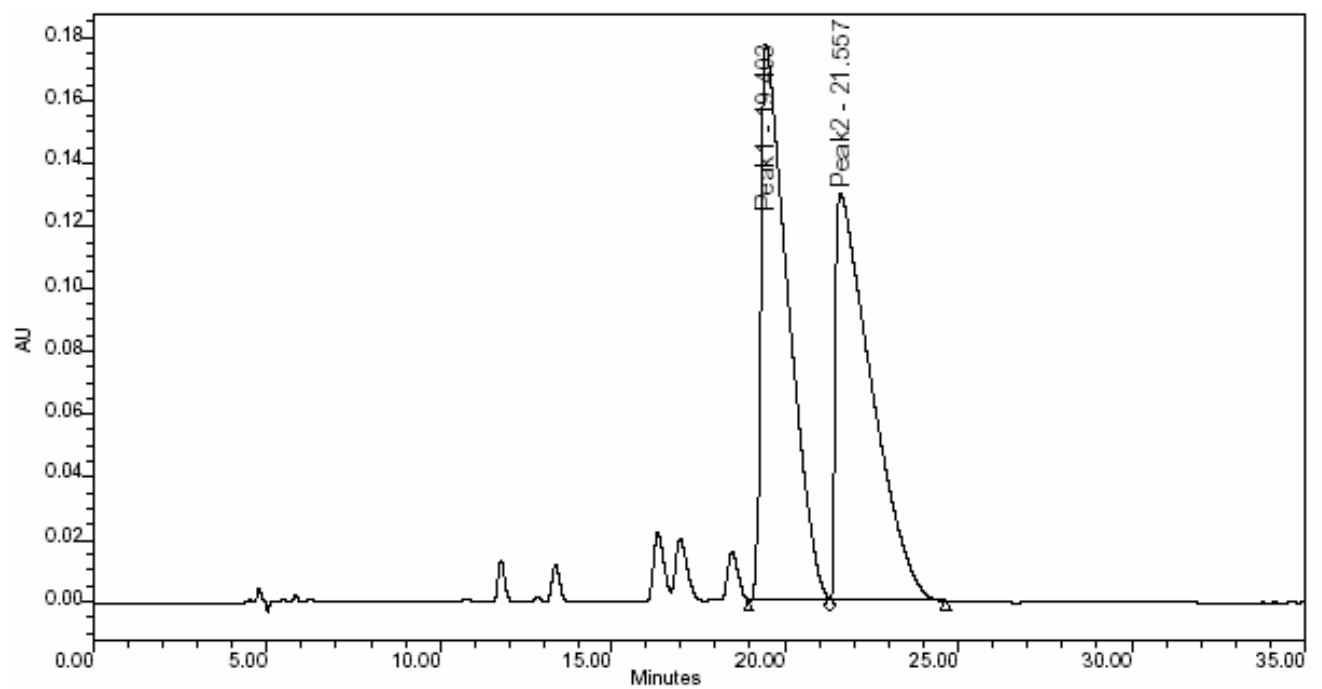

\begin{tabular}{|c|c|c|c|c|c|c|}
\hline & $\begin{array}{c}\text { Peak } \\
\text { Name }\end{array}$ & $\begin{array}{c}\text { RT } \\
(\mathrm{min})\end{array}$ & $\begin{array}{c}\text { Area } \\
\left(\mathrm{V}^{*} \text { sec }\right)\end{array}$ & $\%$ Area & $\begin{array}{c}\text { Height } \\
(\mathrm{V})\end{array}$ & $\begin{array}{c}\% \\
\text { Height }\end{array}$ \\
\hline 1 & Peak1 & 19.403 & 9679061 & 50.83 & 177889 & 57.73 \\
\hline 2 & Peak2 & 21.557 & 9363522 & 49.17 & 130267 & 42.27 \\
\hline
\end{tabular}

\section{Table 2 entry 5}

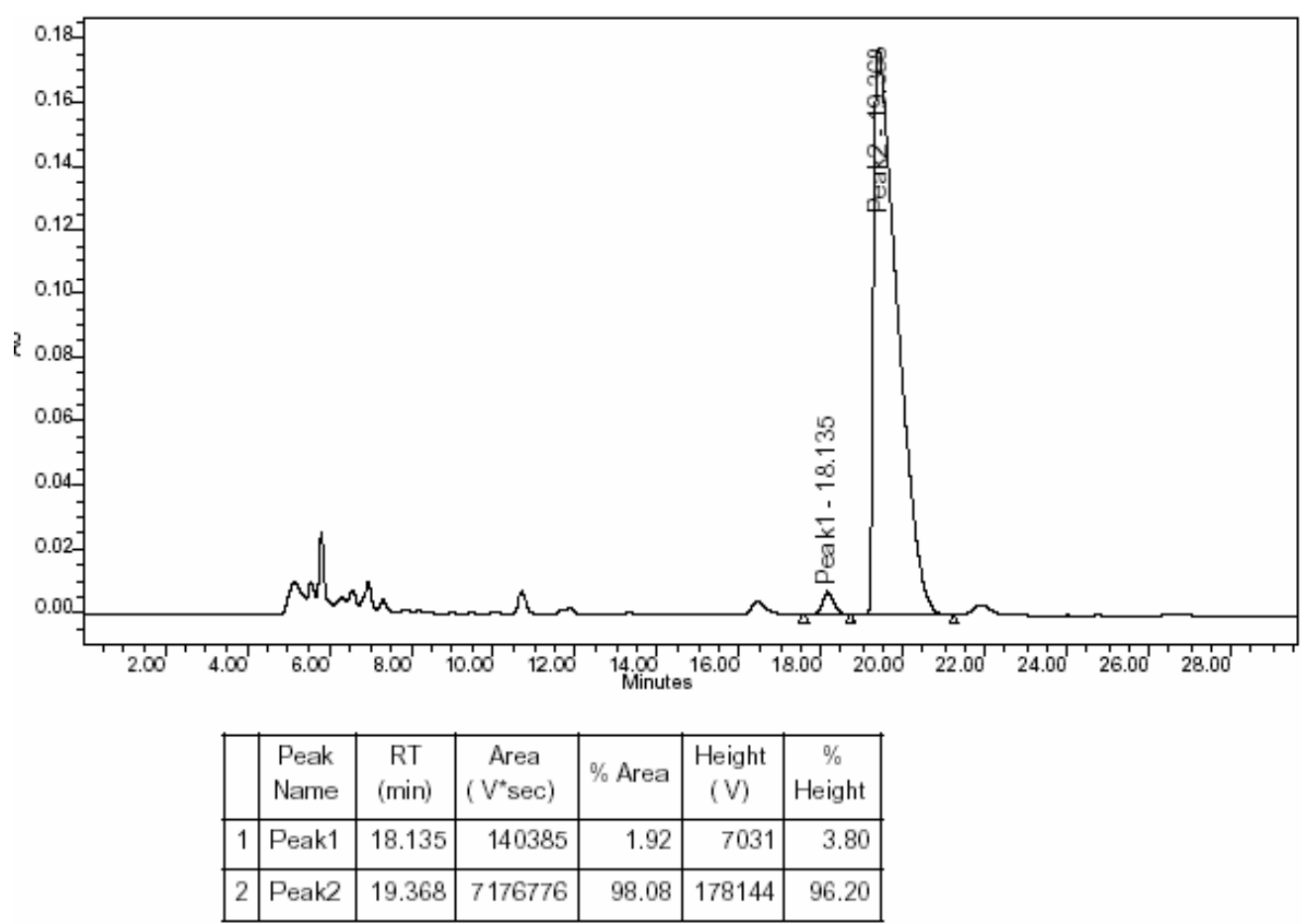


<smiles>CCOC(=O)CC(O)c1ccc(Br)cc1</smiles>

$6 d$ racemate

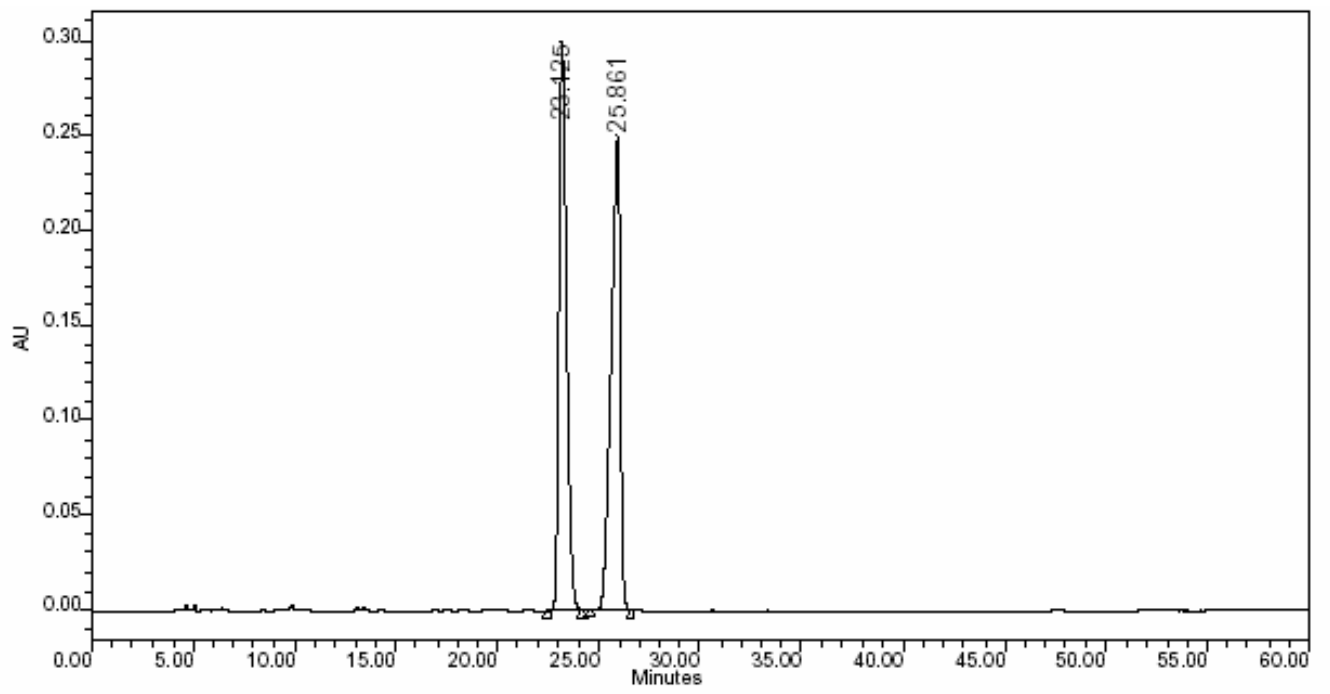

\begin{tabular}{|c|c|c|c|c|c|}
\hline & $\begin{array}{c}\text { RT } \\
(\mathrm{min})\end{array}$ & $\begin{array}{c}\text { Area } \\
\left(\mathrm{V}^{*} \mathrm{sec}\right)\end{array}$ & $\%$ Area & $\begin{array}{c}\text { Height } \\
(\mathrm{V})\end{array}$ & $\begin{array}{c}\% \\
\text { Height }\end{array}$ \\
\hline 1 & 23.125 & 7794911 & 50.04 & 300223 & 54.65 \\
\hline 2 & 25.861 & 7781248 & 49.96 & 249106 & 45.35 \\
\hline
\end{tabular}

\section{Table 2 entry 6}

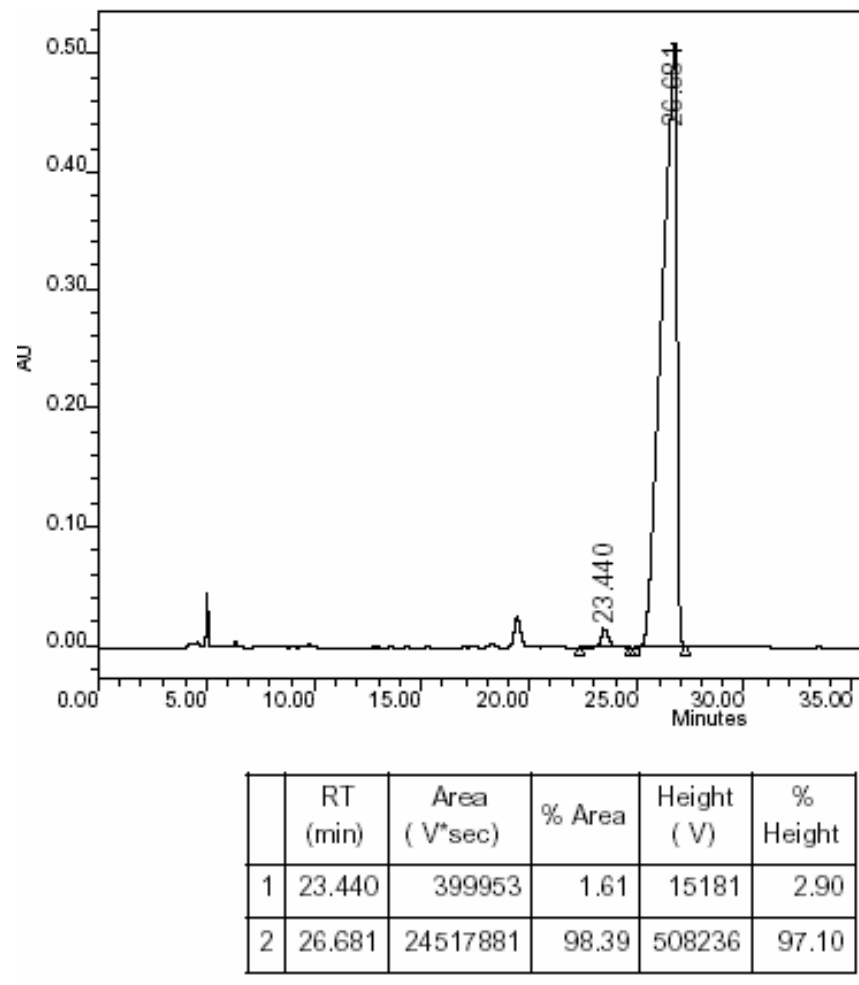


<smiles>CCOC(=O)CC(O)c1ccc(Cl)cc1</smiles>

6 e racemate

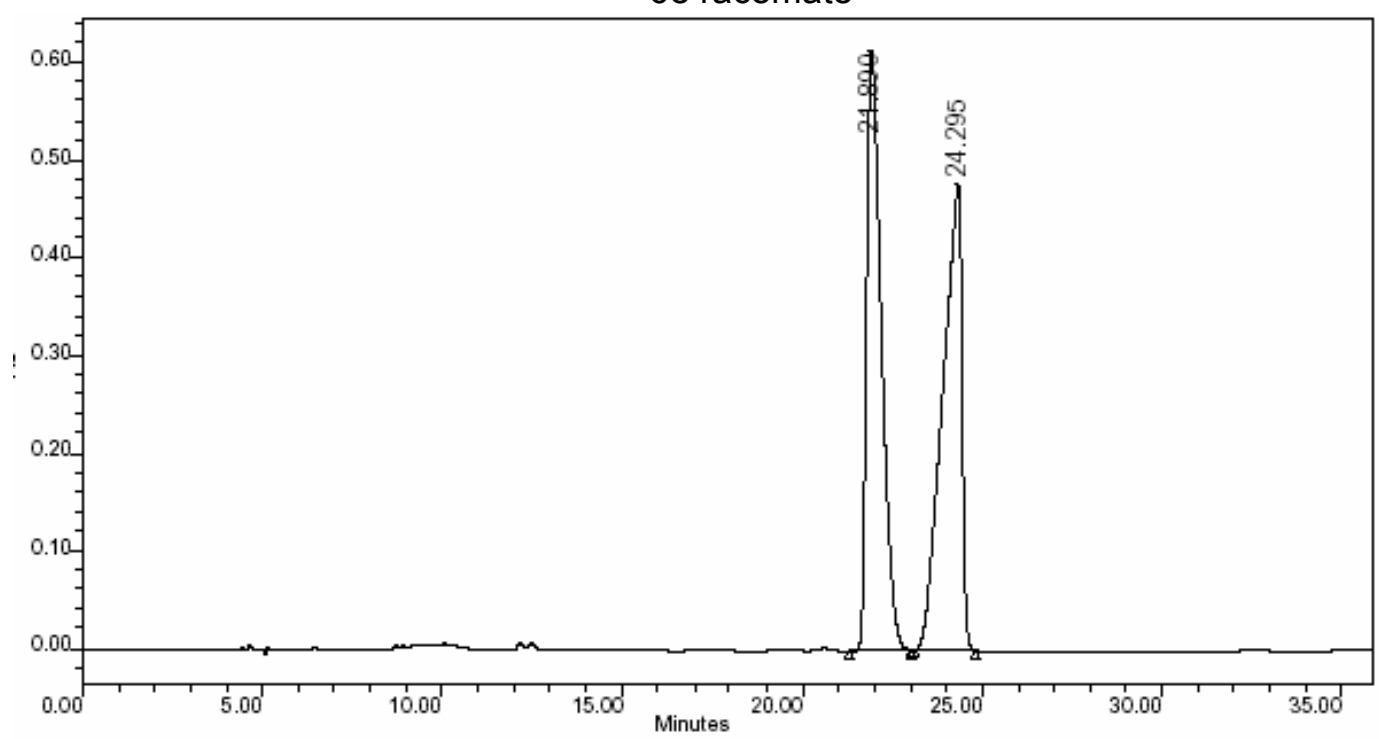

\begin{tabular}{|c|c|c|c|c|c|}
\hline & $\begin{array}{c}\text { RT } \\
(\mathrm{min})\end{array}$ & $\begin{array}{c}\text { Area } \\
\left(\mathrm{V}^{*} \mathrm{sec}\right)\end{array}$ & $\%$ Area & $\begin{array}{c}\text { Height } \\
(\mathrm{V})\end{array}$ & $\begin{array}{c}\% \\
\text { Height }\end{array}$ \\
\hline 1 & 21.890 & 17598901 & 49.91 & 615913 & 56.35 \\
\hline 2 & 24.295 & 17661107 & 50.09 & 477178 & 43.65 \\
\hline
\end{tabular}

Table 2 entry 7

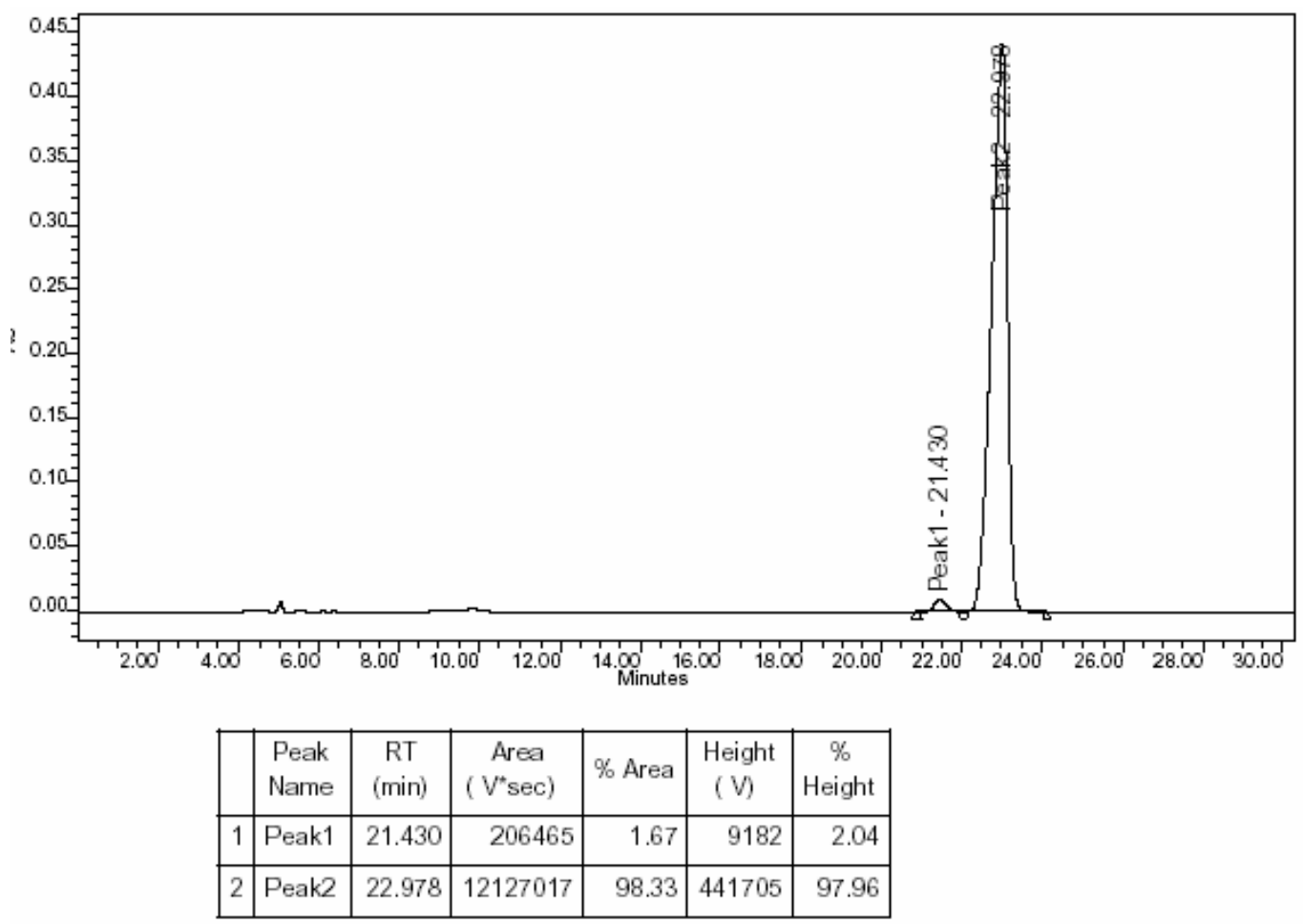



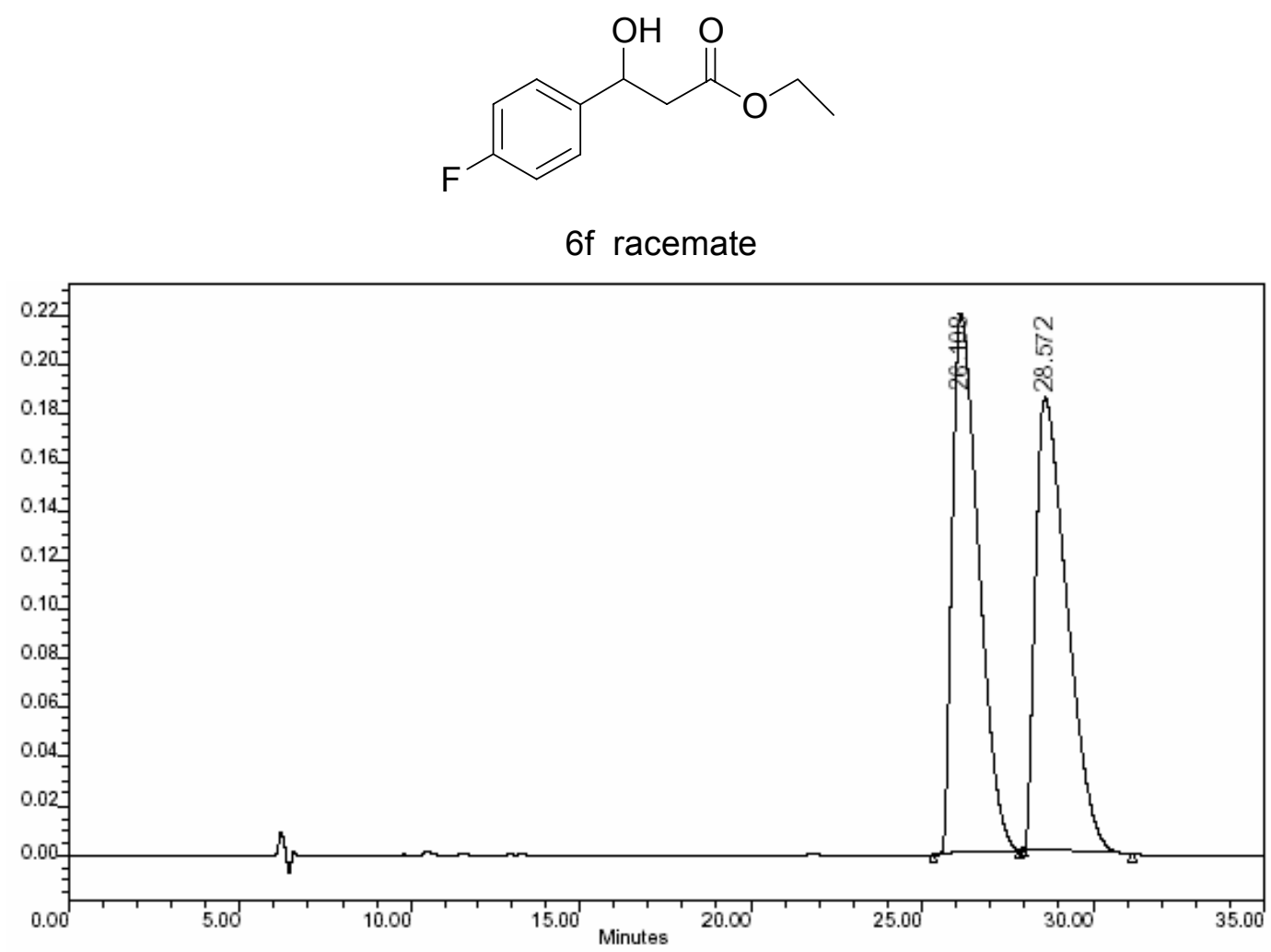

\begin{tabular}{|c|c|c|c|c|c|}
\hline & $\begin{array}{c}\text { RT } \\
(\mathrm{min})\end{array}$ & $\begin{array}{c}\text { Area } \\
\left(\mathrm{V}^{*} \mathrm{sec}\right)\end{array}$ & $\%$ Area & $\begin{array}{c}\text { Height } \\
(\mathrm{V})\end{array}$ & $\begin{array}{c}\% \\
\text { Height }\end{array}$ \\
\hline 1 & 26.108 & 11872930 & 50.07 & 220477 & 54.50 \\
\hline 2 & 28.572 & 11841500 & 49.93 & 184087 & 45.50 \\
\hline
\end{tabular}

Table 2 entry 8

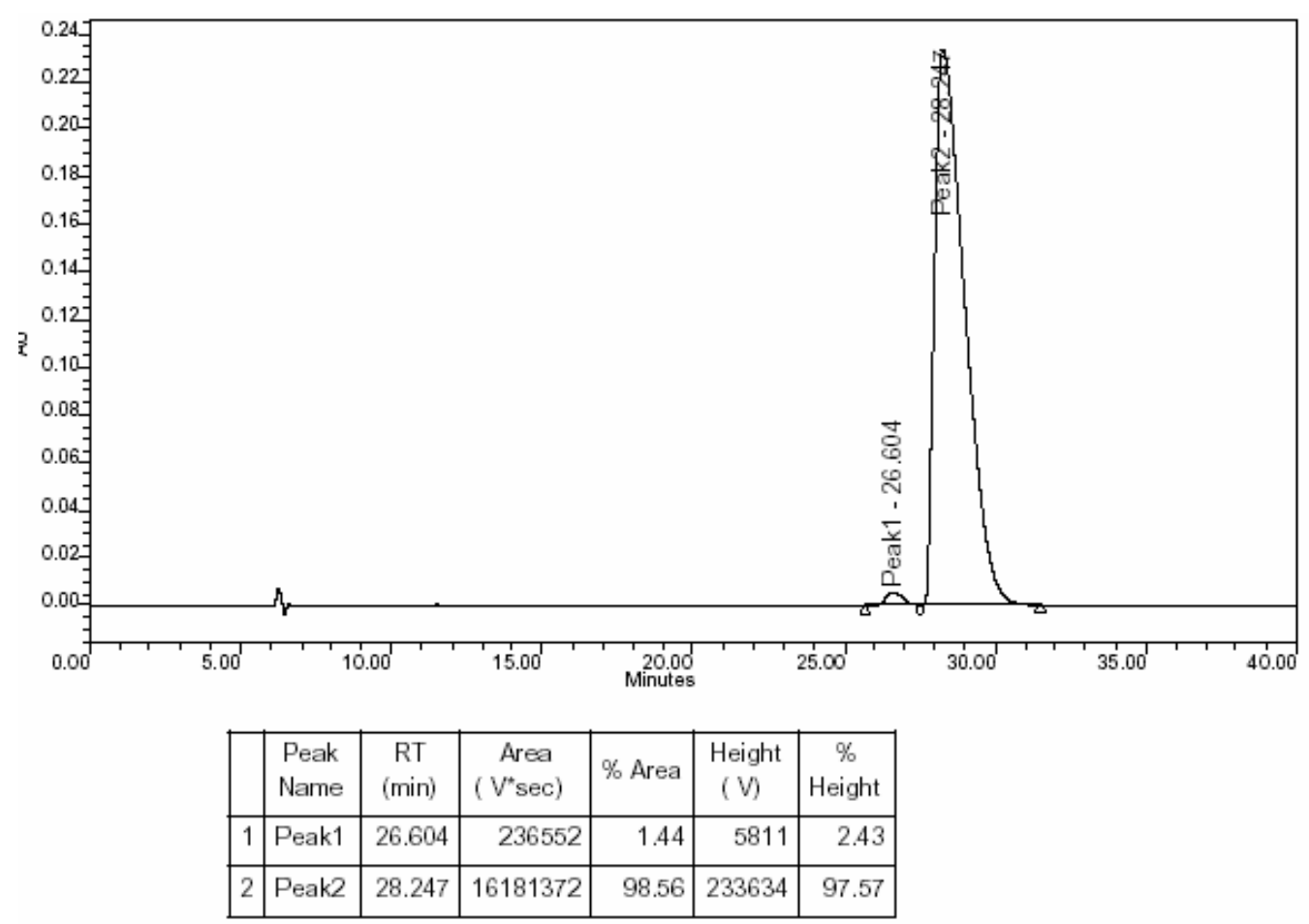




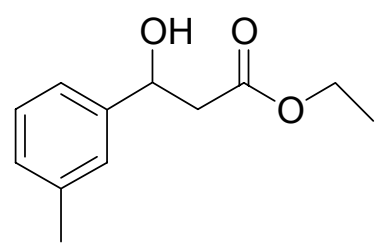

$6 \mathrm{~g}$ racemate

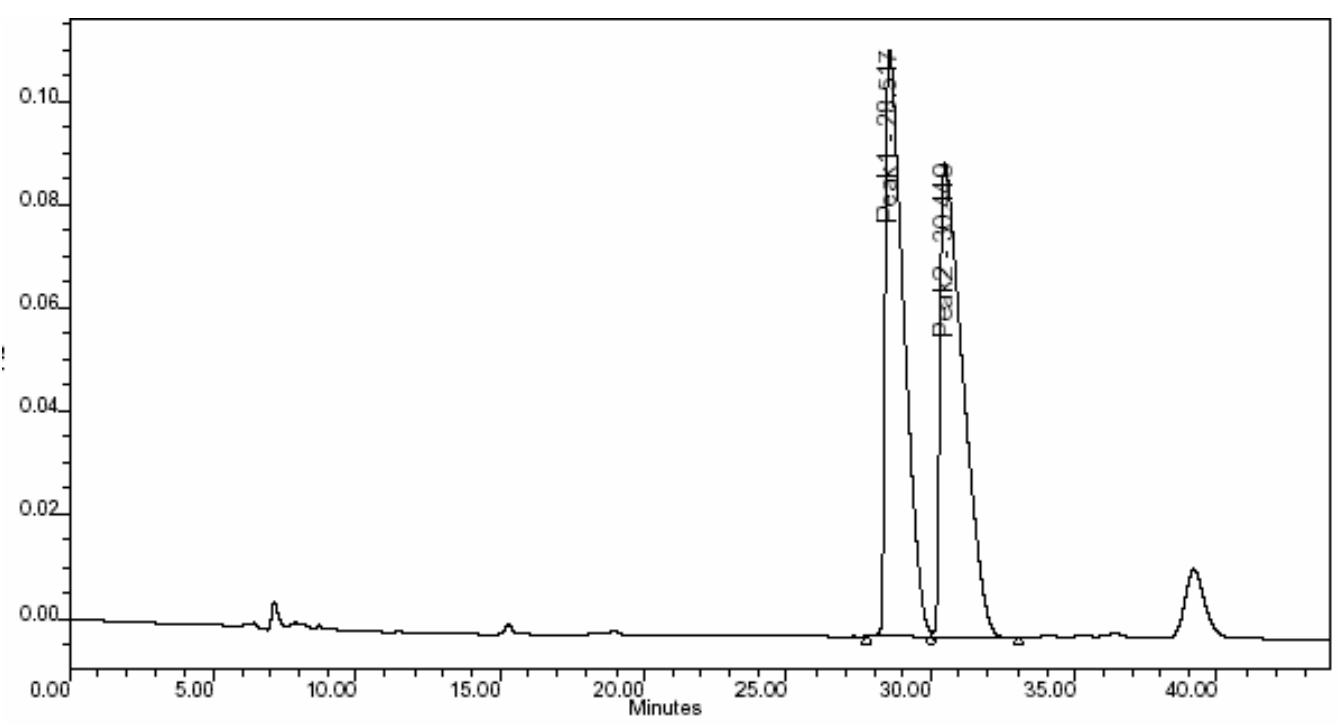

\begin{tabular}{|c|c|c|c|c|c|c|}
\hline & $\begin{array}{c}\text { Peak } \\
\text { Name }\end{array}$ & $\begin{array}{c}\text { RT } \\
(\mathrm{min})\end{array}$ & $\begin{array}{c}\text { Area } \\
\left(\mathrm{V}^{*} \text { sec }\right)\end{array}$ & $\%$ Area & $\begin{array}{c}\text { Height } \\
(\mathrm{V})\end{array}$ & $\begin{array}{c}\% \\
\text { Height }\end{array}$ \\
\hline 1 & Peak1 & 28.517 & 5083019 & 50.00 & 113418 & 55.37 \\
\hline 2 & Peak2 & 30.449 & 5083729 & 50.00 & 91423 & 44.63 \\
\hline
\end{tabular}

\section{Table2 entry 9}

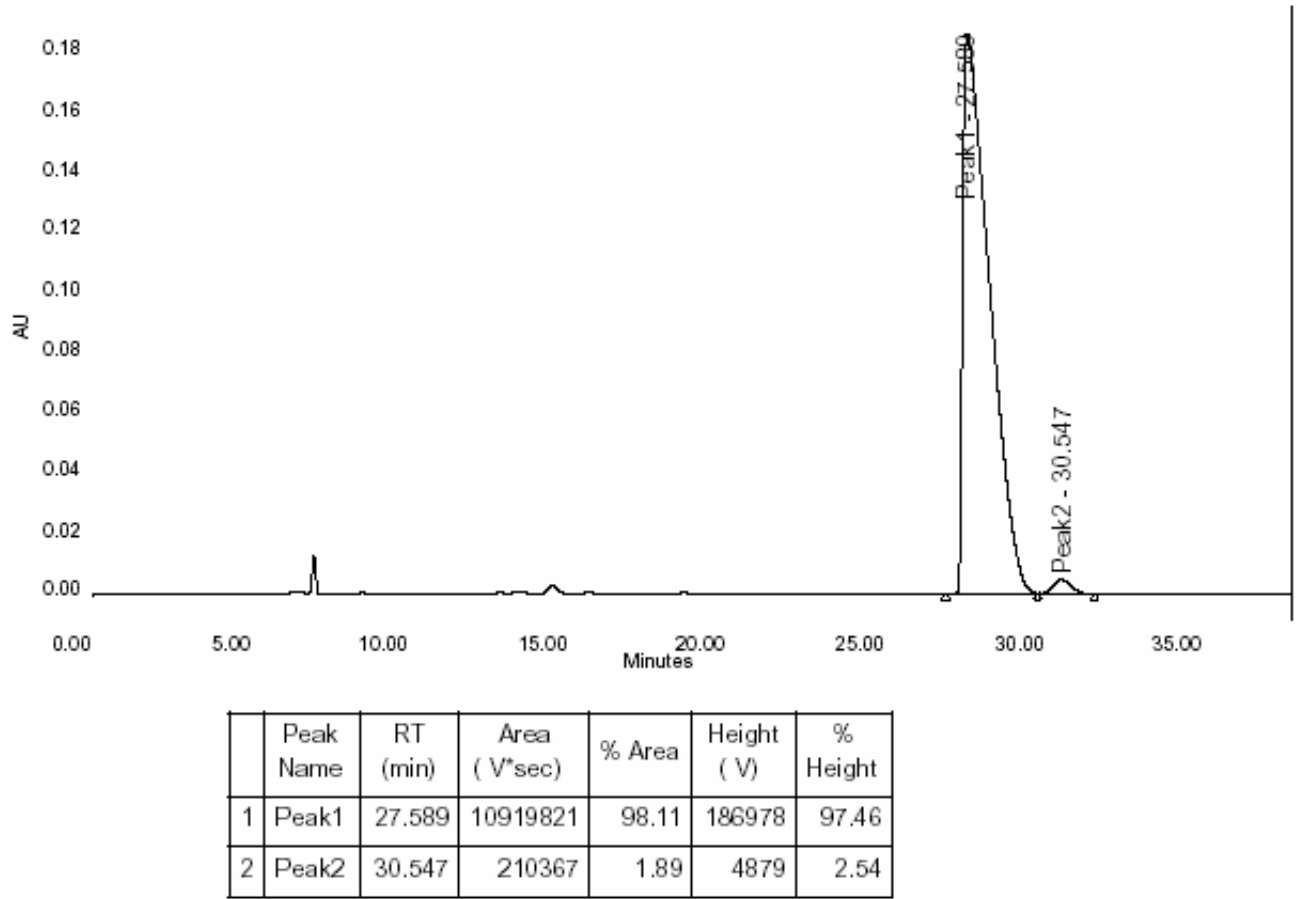


<smiles>CCOC(=O)CC(O)c1cccc(Cl)c1</smiles>

$6 h$ racemate

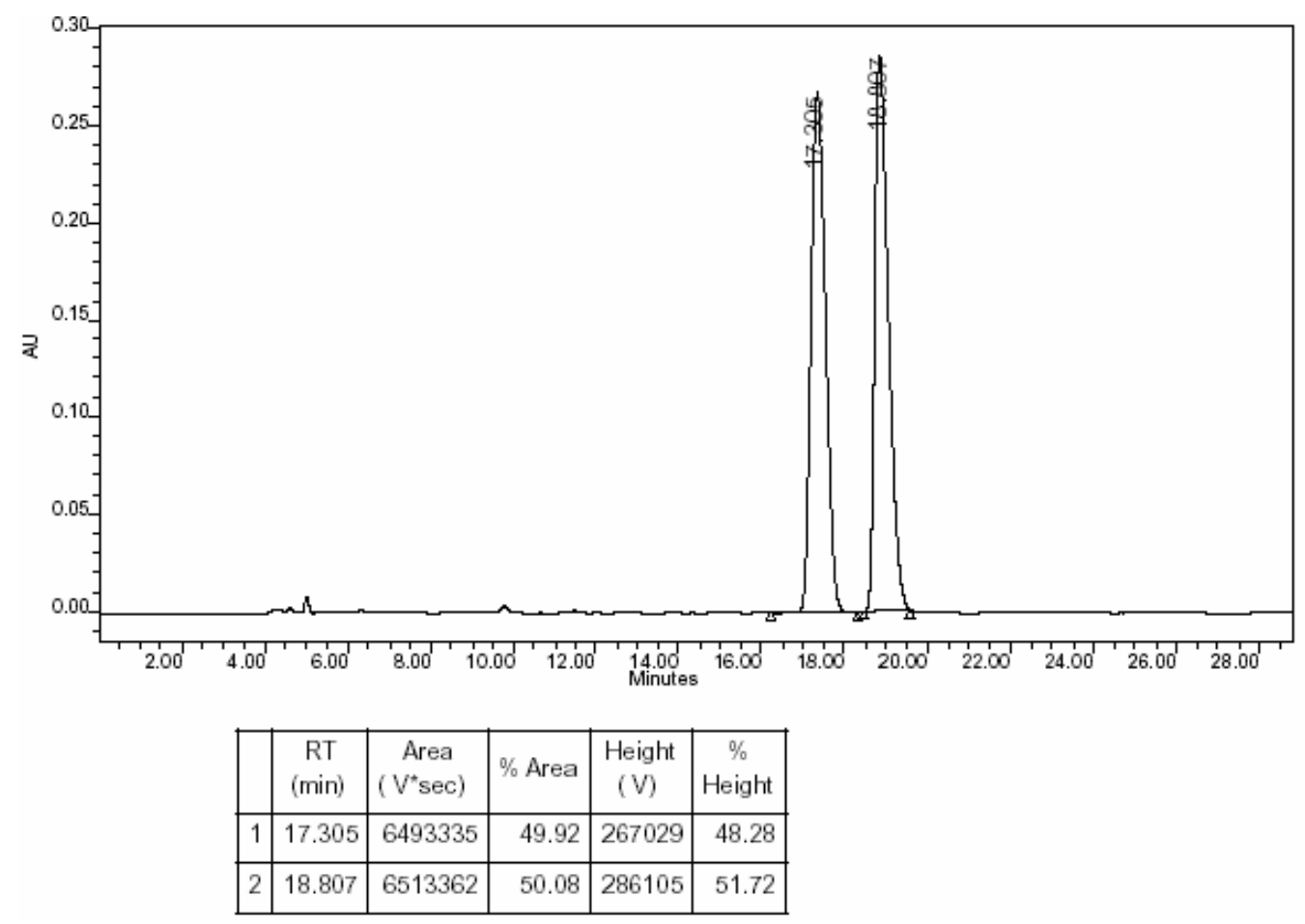

Table 2 entry 10

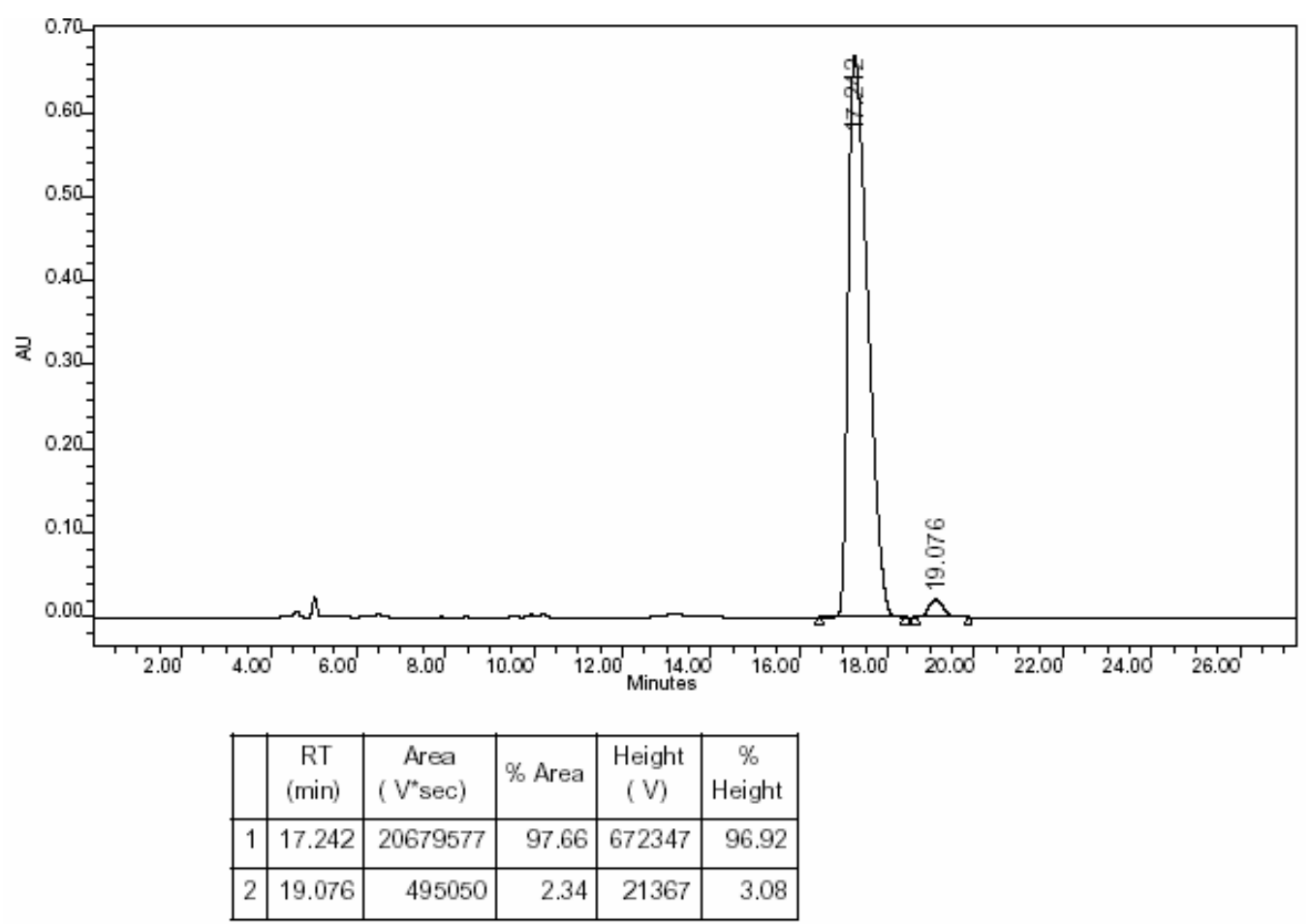


<smiles>CCOC(=O)CC(O)c1ccccc1C</smiles>

\section{$6 i$ racemate}

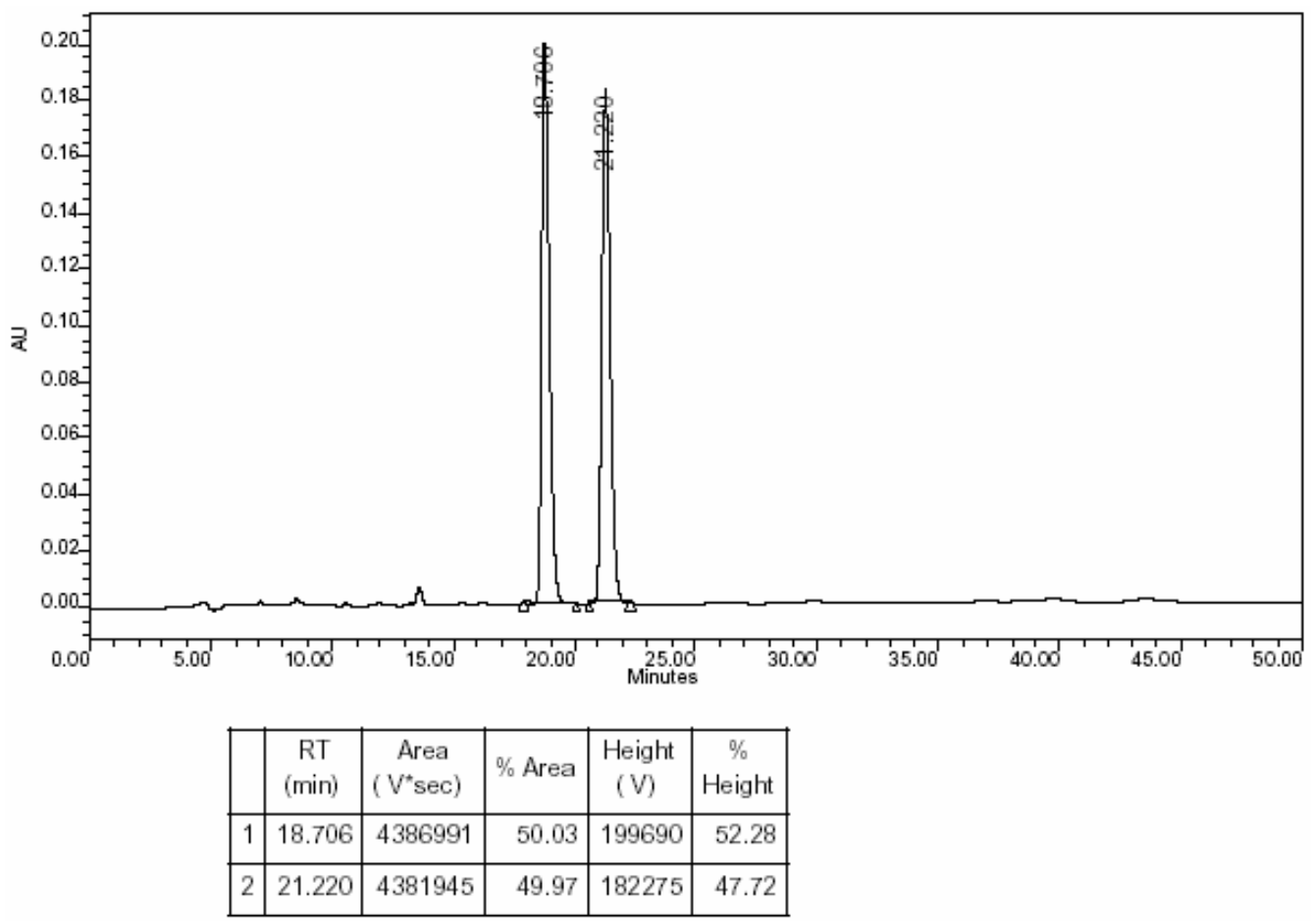

Table2 entry 11

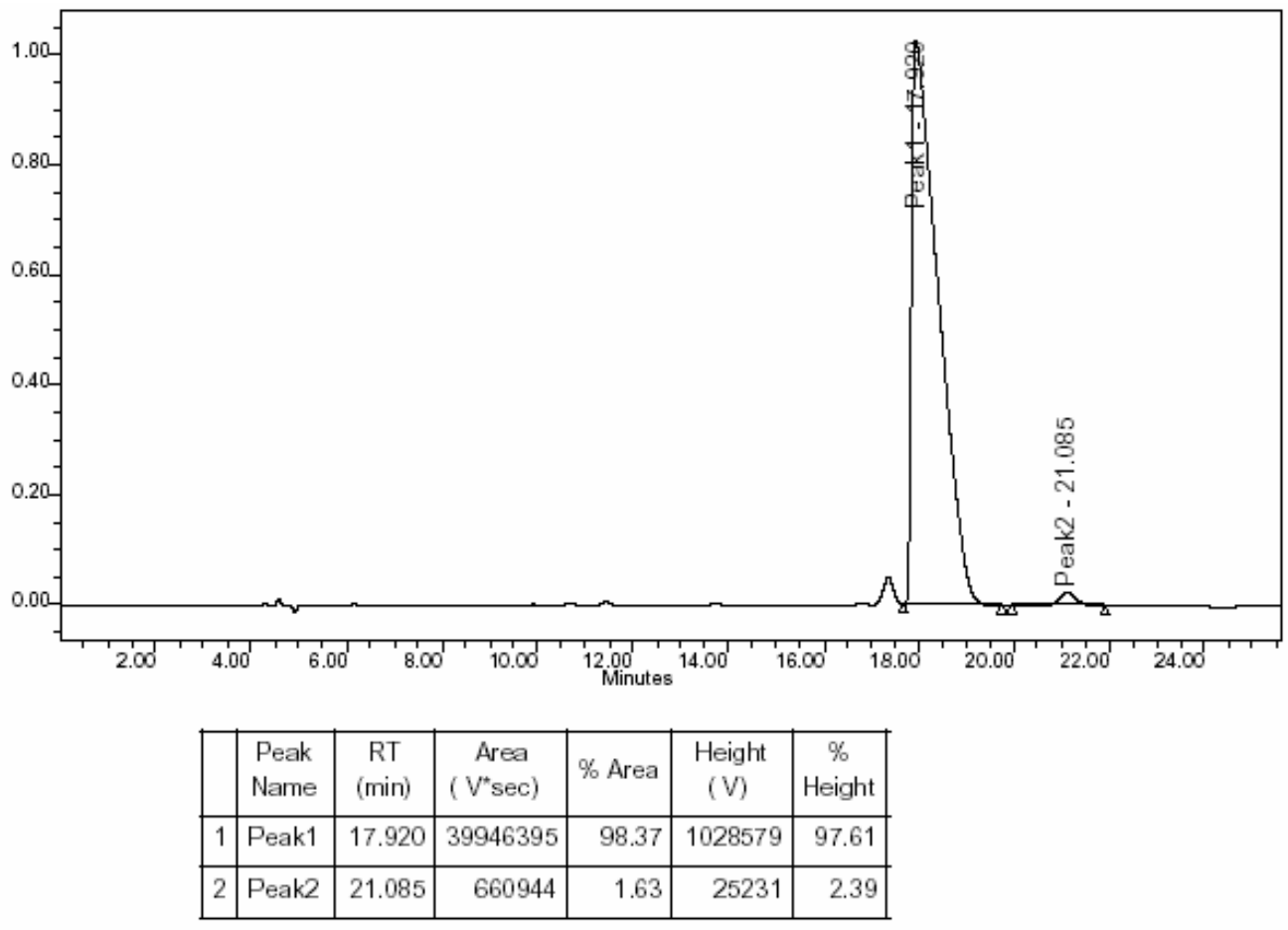




\section{Table3 entry 1}

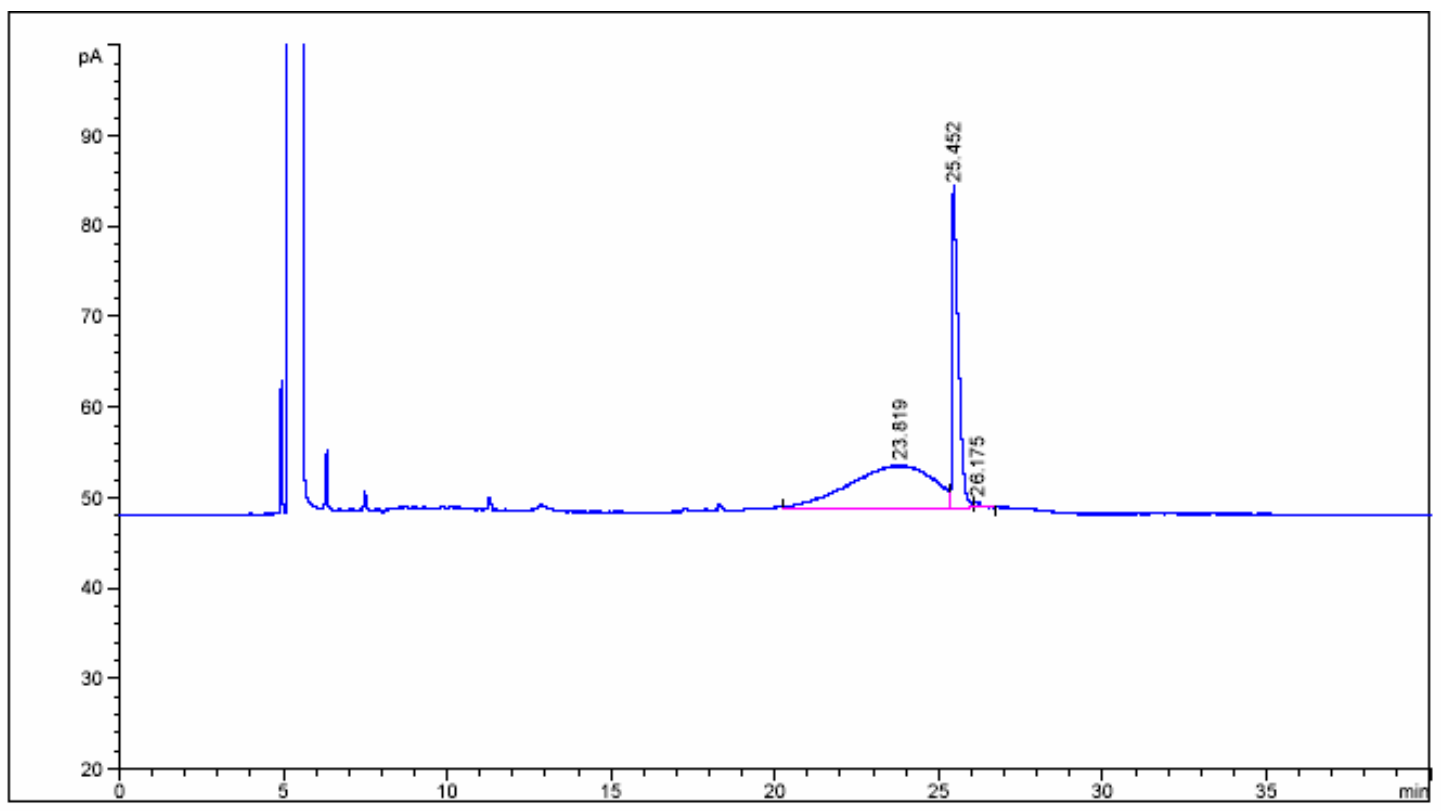

Area Percent Report

Sorted By

Multiplier

Dilution

Sample Amount

Signal

1.0000

1.0000

1.00000 [ng/ul] (not used in calc.)

Signal 1: FIDI A,

\begin{tabular}{|c|c|c|c|c|c|c|}
\hline $\begin{array}{c}\text { Peak } \\
\text { \# }\end{array}$ & $\begin{array}{l}\text { RetTime } \\
\text { [min] }\end{array}$ & Type & $\begin{array}{l}\text { Width } \\
\text { [min] }\end{array}$ & $\begin{array}{r}\text { Area } \\
{\left[p^{*}{ }^{*} s\right]}\end{array}$ & $\begin{array}{l}\text { Height } \\
\text { [pA] }\end{array}$ & $\begin{array}{c}\text { Area } \\
\text { g }\end{array}$ \\
\hline 1 & 23.819 & BV & 2.0 & 823.38721 & & 090 \\
\hline 2 & 25.452 & vV & 0.1693 & 452.69266 & 35.37795 & 35.22574 \\
\hline 3 & 26.175 & VP & 0.1670 & 9.03897 & $6.98604 e-1$ & 0.70336 \\
\hline
\end{tabular}

Totals :

$1285.11883 \quad 40.75364$

Results obtained with enhanced integrator!

$\star \star *$ End of Report *** 


\section{Table 3 entry 2}

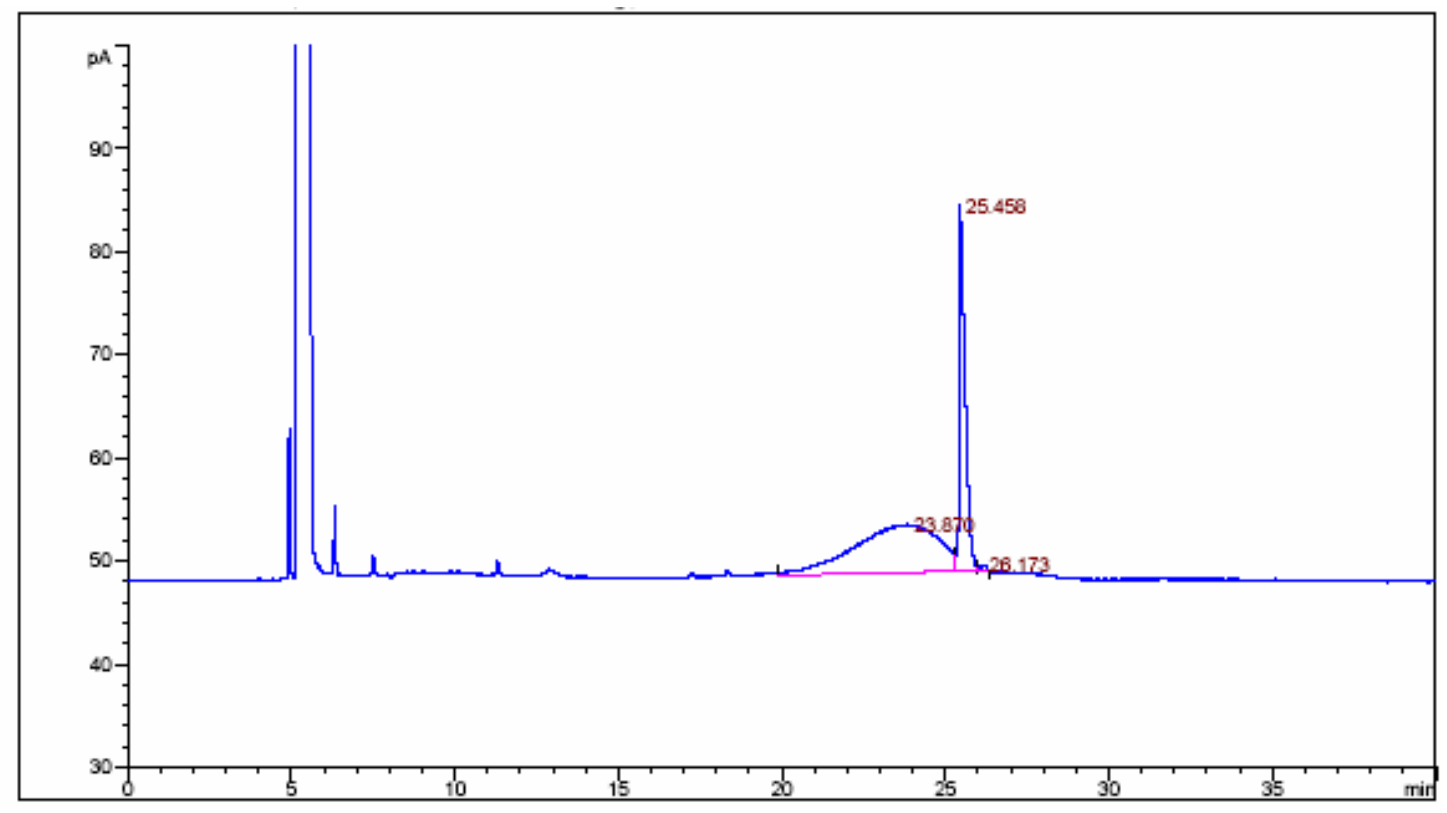

Area Percent Report

sorted By

Calib. Deta Modified

Multiplier

Dilution

Sample Amount
Signal

02-12-10 10:54:30下午

1.0000

1.00000 [ng/ul) (not used in calc.)

Signal 1: FID1 $\lambda_{\text {, }}$

\begin{tabular}{|c|c|c|c|c|c|c|}
\hline $\begin{array}{c}\text { Peak } \\
\hbar\end{array}$ & $\begin{array}{l}\text { RetTime } \\
\text { [min] }\end{array}$ & Type & $\begin{array}{l}\text { width } \\
\text { [min] }\end{array}$ & $\begin{array}{r}\text { Area } \\
\left.\text { [p }{ }^{*}{ }^{*}\right]\end{array}$ & $\begin{array}{c}\text { Area } \\
\text { g }\end{array}$ & Name \\
\hline 1 & 2.021 & & 0.0000 & 0.00000 & 0.00000 & \\
\hline 2 & 2.131 & & 0.0000 & 0.00000 & 0.00000 & \\
\hline 3 & 2.271 & & 0.0000 & 0.00000 & 0.00000 & \\
\hline 4 & 2.353 & & 0.0000 & 0.00000 & 0.00000 & \\
\hline 5 & 2.513 & & 0.0000 & 0.00000 & 0.00000 & \\
\hline 6 & 2.626 & & 0.0000 & 0.00000 & 0.00000 & \\
\hline 3 & 2.698 & & 0.0000 & 0.00000 & 0.00000 & \\
\hline 8 & 3.026 & & 0.0000 & 0.00000 & 0.00000 & \\
\hline 9 & 23.870 & $M$ & 2.9755 & 822.28564 & 64.23015 & ? \\
\hline 10 & 25.458 & $M$ & 0.2129 & 449.47134 & 35.13084 & ? \\
\hline 11 & 26.173 & $M$ & 0.2245 & 7.66383 & 0.59901 & $?$ \\
\hline
\end{tabular}




\section{Table 3 entry3}

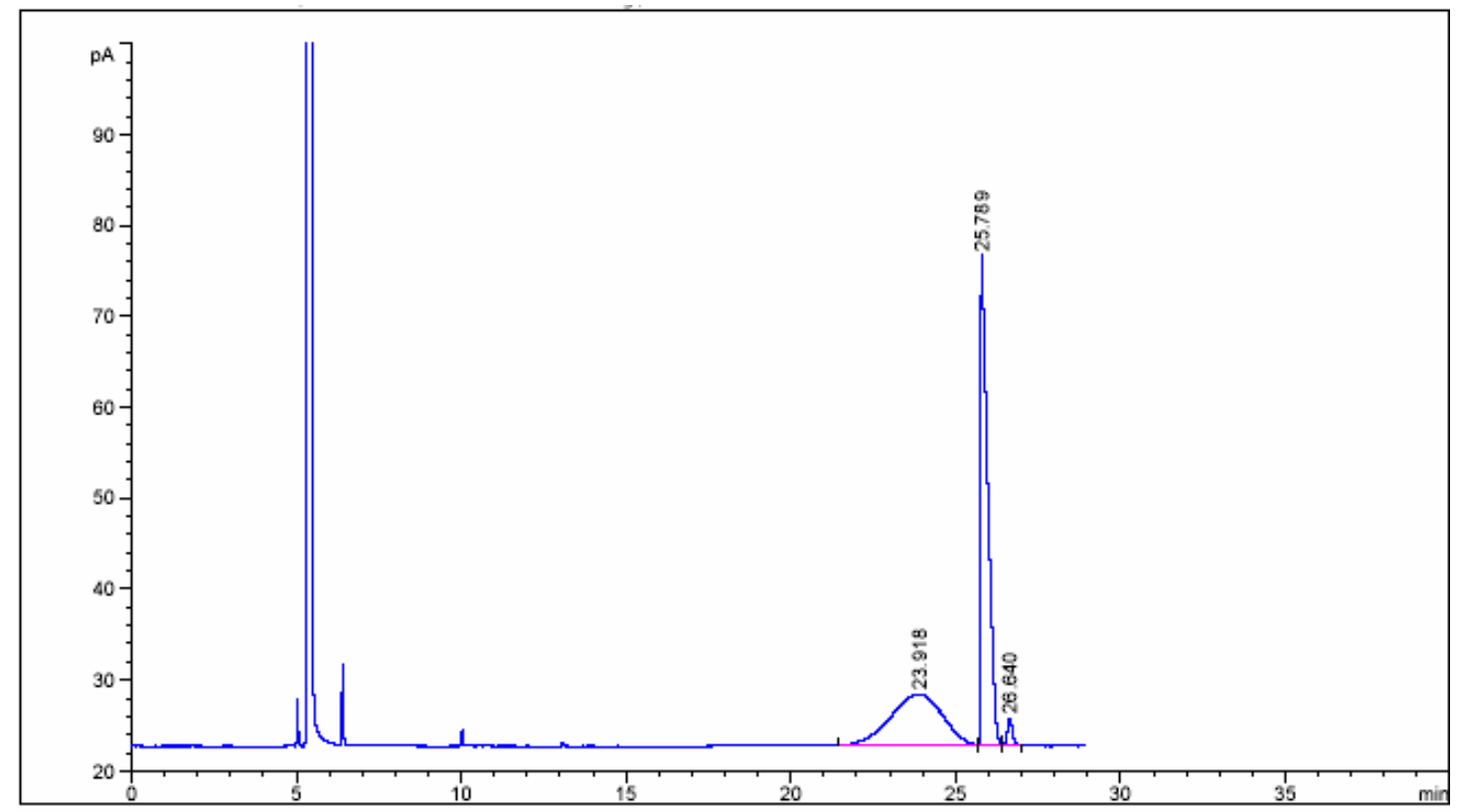

Area Percent Report

Sorted By

Multiplier

Dilution

Sample Amount

Signal

1.0000

1. 0000

1.00000 [ng/ul] (not used in calc.)

Signal 1: FID1 A,

\begin{tabular}{|c|c|c|c|c|c|c|}
\hline$\underset{\#}{\text { eak }}$ & $\begin{array}{l}\text { RetTime } \\
\text { [min] }\end{array}$ & Type & $\begin{array}{l}\text { Width } \\
\text { [min] }\end{array}$ & $\begin{array}{r}\text { Area } \\
{\left[\mathrm{pA}{ }^{*} \mathrm{~s}\right]}\end{array}$ & $\begin{array}{l}\text { Height } \\
\text { [pA] }\end{array}$ & $\begin{array}{c}\text { Area } \\
8\end{array}$ \\
\hline 1 & & BV & & 621 & & 43 \\
\hline 2 & & VV & & 785.51544 & 53.66879 & 54.50845 \\
\hline 3 & 26.640 & VB & 0.1706 & 34.04292 & 2.99452 & 2.36230 \\
\hline
\end{tabular}

Totals :

1441.08931

62.28998

Results obtained with enhanced integrator!

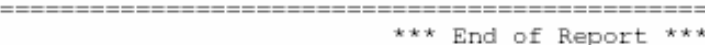




\section{Table 3 entry 4}

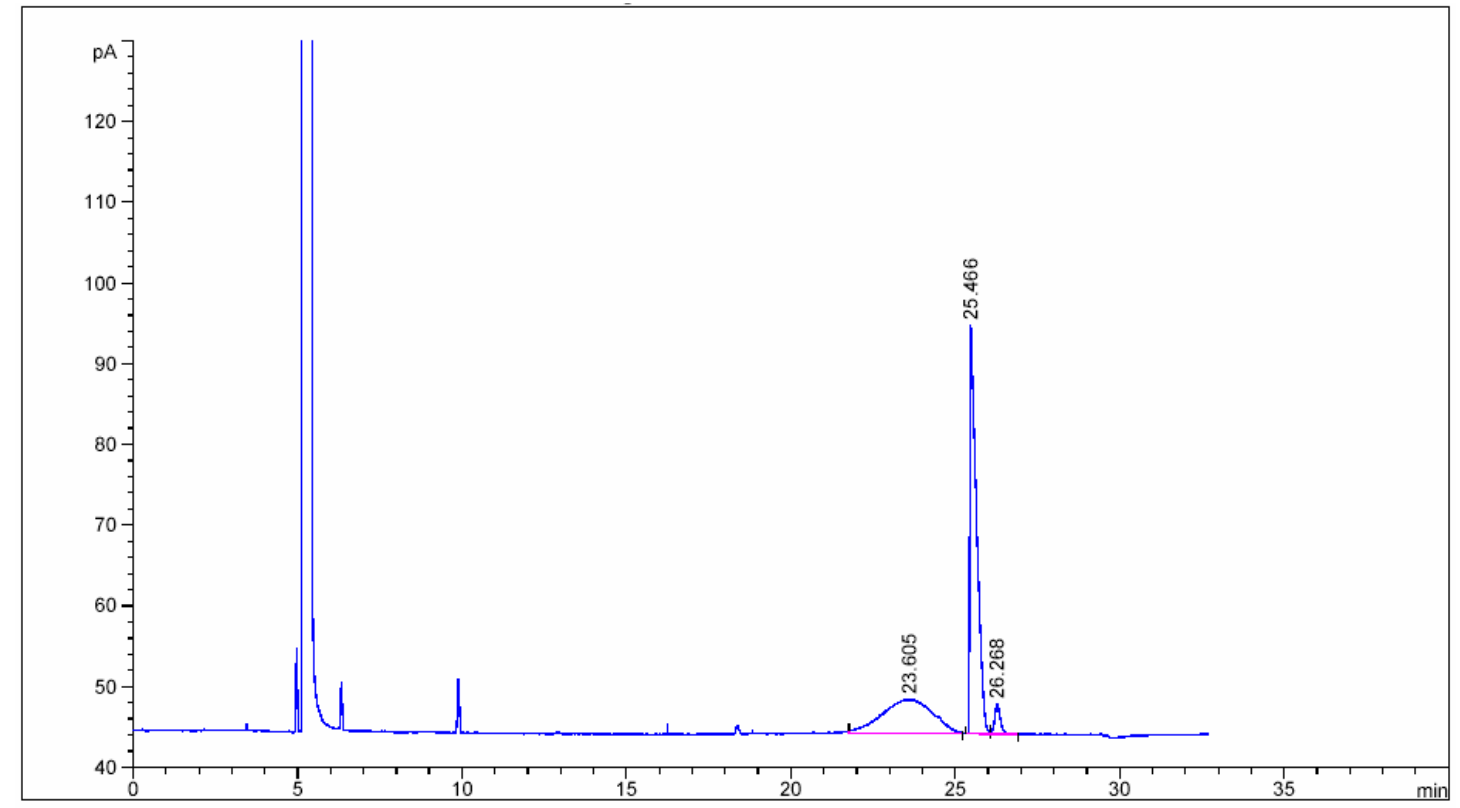

Area Percent Report

Sorted By

Multiplier

Dilution

Sample Amount

Signal

1.0000

1.0000

1.00000 [ng/ul] (not used in calc.)

Signal 1: FID1 A,

\begin{tabular}{|c|c|c|c|c|c|c|}
\hline $\begin{array}{c}\text { Peak } \\
\#\end{array}$ & $\begin{array}{c}\text { RetTime } \\
\text { [min] }\end{array}$ & Type & $\begin{array}{l}\text { Width } \\
\text { [min] }\end{array}$ & $\begin{array}{r}\text { Area } \\
{\left[\mathrm{pA}^{*} \mathrm{~s}\right]}\end{array}$ & $\begin{array}{l}\text { Height } \\
{[\mathrm{pA}]}\end{array}$ & $\begin{array}{c}\text { Area } \\
\frac{a}{5}\end{array}$ \\
\hline 1 & & & & 453. & & \\
\hline 2 & 25.466 & VB & 0.1799 & 711.70294 & 50.66411 & 58.87148 \\
\hline 3 & 26.268 & $\mathrm{BB}$ & 0.1756 & 43.87442 & 3.72063 & 3.62926 \\
\hline
\end{tabular}

Totals :

$1208.90964 \quad 58.61137$

Results obtained with enhanced integrator!

$\star \star * *$ End of Report $* \star * *$ 


\section{Table 3 entry 5}

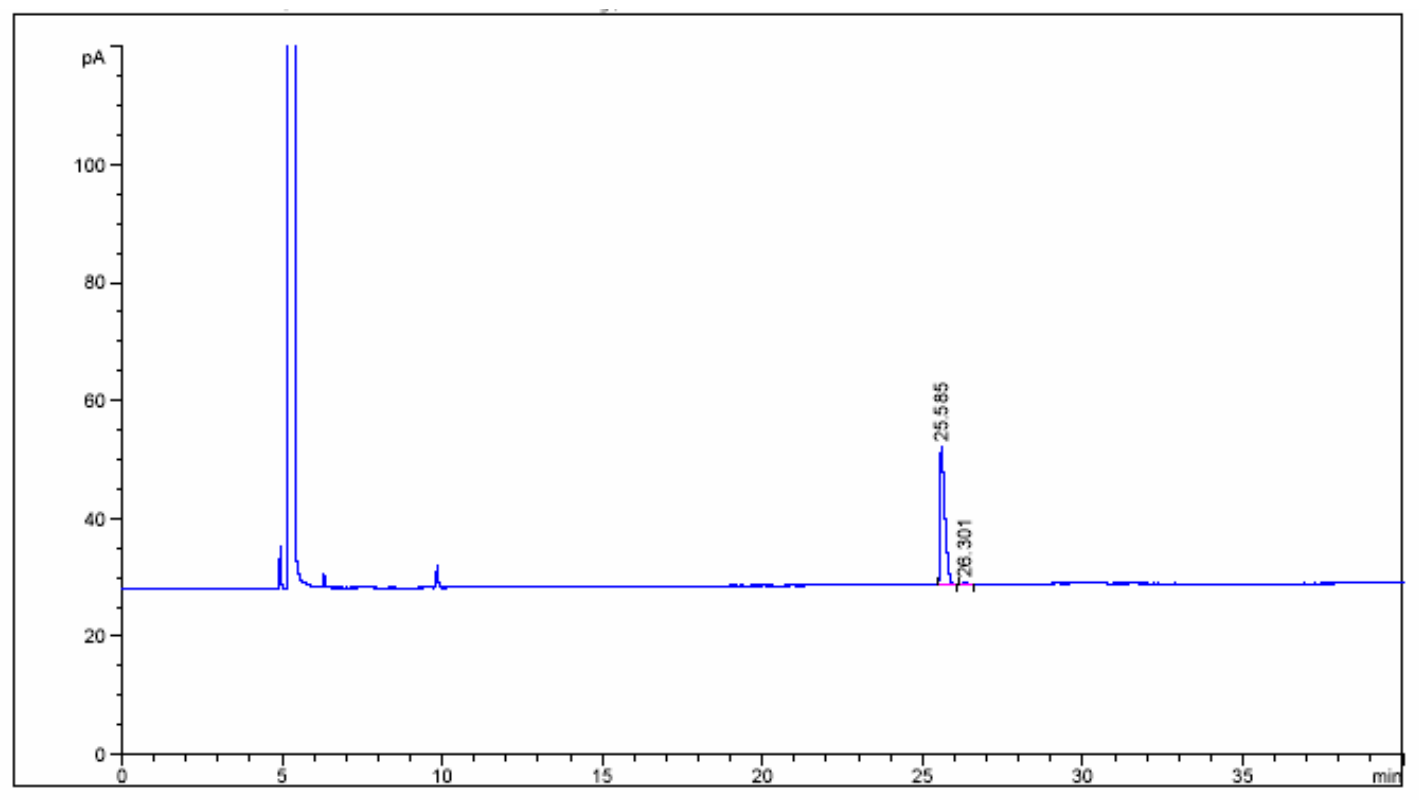

Area Percent Report

\begin{tabular}{|c|c|c|c|c|}
\hline Sorted By & : & Signal & & \\
\hline Multiplier & : & 1.0000 & & \\
\hline Dilution & $:$ & 1.0000 & & \\
\hline Sample Amount & : & 1.00000 & {$[\mathrm{ng} / \mathrm{u} 1]$} & (not used in calc.) \\
\hline \multicolumn{5}{|l|}{ Signal 1: FID1 A, } \\
\hline $\begin{array}{c}\text { Peak RetTime Type } \\
\# \quad[\text { min] }\end{array}$ & $\begin{array}{l}\text { Width } \\
\text { [min] }\end{array}$ & $\begin{array}{r}\text { Area } \\
{\left[\mathrm{pA}^{\star} \mathrm{s}\right]}\end{array}$ & $\begin{array}{l}\text { Height } \\
{[\mathrm{pA}]}\end{array}$ & $\begin{array}{c}\text { Area } \\
8\end{array}$ \\
\hline $125.585 \mathrm{~PB}$ & 0.1428 & 242.47488 & $\mid-10$. & 98.42145 \\
\hline $226.301 \mathrm{PP}$ & 0.1341 & 3.88898 & $4.04707 e-1$ & 1.57855 \\
\hline Totals : & & 246.36386 & 23.81568 & \\
\hline
\end{tabular}

$\star * *$ End of Report *** 


\section{Table3 entry6}

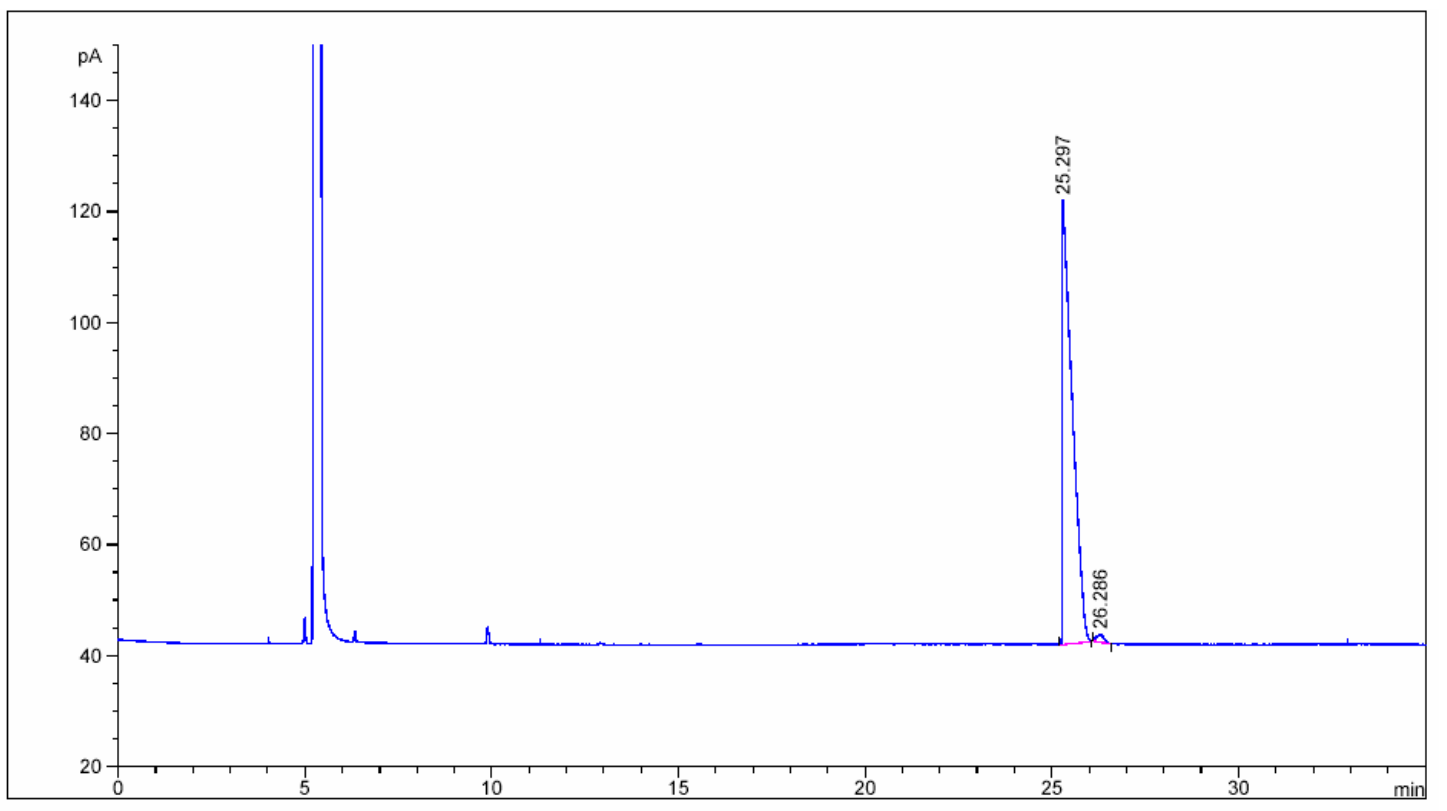

Area Percent Report

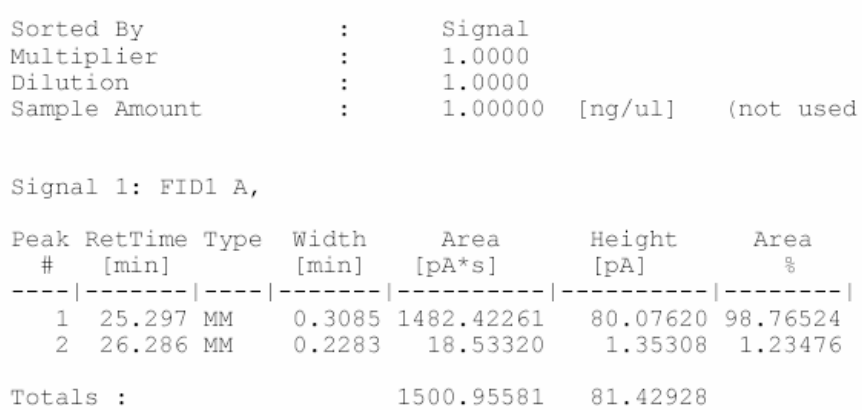

Totals :

1500.95581

31.42928

Results obtained with enhanced integrator!

*** End of Report *** 